Pacific

Journal of

Mathematics

HANKEL OPERATORS OVER COMPLEX MANIFOLDS

Thomas Deck and Leonard Gross

Volume $205 \quad$ No. 1

July 2002 


\title{
HANKEL OPERATORS OVER COMPLEX MANIFOLDS
}

\author{
Thomas Deck And LeOnard Gross
}

\begin{abstract}
Given a complex manifold $M$ endowed with a hermitian metric $g$ and supporting a smooth probability measure $\mu$, there is a naturally associated Dirichlet form operator $A$ on $L^{2}(\mu)$. If $b$ is a function in $L^{2}(\mu)$ there is a naturally associated Hankel operator $\boldsymbol{H}_{b}$ defined in holomorphic function spaces over $M$. We establish a relation between hypercontractivity properties of the semigroup $e^{-t A}$ and boundedness, compactness and trace ideal properties of the Hankel operator $\boldsymbol{H}_{b}$. Moreover there is a natural algebra $\mathcal{R}$ of holomorphic functions on $M$, analogous to the algebra of holomorphic polynomials on $\mathbb{C}^{m}$, and which is determined by the spectral subspaces of $A$. We explore the relation between the algebra $\mathcal{R}$ and the Hilbert-Schmidt character of the Hankel operator $H_{b}$. We also show that the reproducing kernel is very well related to the operator $A$.
\end{abstract}

\section{Introduction.}

Let $M$ be a complex manifold of finite complex dimension $d$ and let $\mu$ be a probability measure on $M$ having a continuous, strictly positive density in each coordinate chart. The set of holomorphic functions on $M$ which are square integrable with respect to $\mu$ is then a Hilbert space, which we denote by $\mathcal{H} L^{2}(\mu)$. By a Hankel form on $\mathcal{H} L^{2}(\mu)$ we mean a jointly continuous bilinear form $\Gamma: \mathcal{H} L^{2}(\mu) \times \mathcal{H} L^{2}(\mu) \rightarrow \mathbb{C}$ such that

$$
\Gamma(f, g)=\Gamma(f g, 1) \quad \text { for all } f, g \in \mathcal{R},
$$

where $\mathcal{R}$ is some dense subspace of $\mathcal{H} L^{2}(\mu)$ for which $f g \in \mathcal{H} L^{2}(\mu)$ whenever $f$ and $g$ are in $\mathcal{R}$. This allows (1.1) to make sense. To this end we will use for $\mathcal{R}$ a naturally arising algebra of functions containing the constants. Since $f \mapsto \Gamma(f, 1)$ is a continuous linear functional on $\mathcal{H} L^{2}(\mu)$ there is a unique element $b \in \mathcal{H} L^{2}(\mu)$ such that $\Gamma(f, 1)=\int_{M} \bar{b} f d \mu$. Then (1.1) implies

$$
\Gamma(f, g)=\int_{M} \bar{b} f g d \mu \quad \text { for all } f, g \in \mathcal{R} .
$$

By continuity this representation determines the given Hankel form $\Gamma$ uniquely on $\mathcal{H} L^{2}(\mu)$. 
Any measurable function $b$ on $M$ which determines a Hankel form by (1.2) will be called a symbol of $\Gamma$. Notice that when $b \in L^{2}(\mu)$ is a symbol of a Hankel form $\Gamma$, and $P: L^{2}(\mu) \rightarrow \mathcal{H} L^{2}(\mu)$ is the orthogonal projection, then $P b$ is the unique symbol of $\Gamma$ in $\mathcal{H} L^{2}(\mu)$. The Hankel form determined by $b$ as in (1.2) will be denoted $\Gamma_{b}$.

We will write $\langle f, g\rangle=\int_{M} f \bar{g} d \mu$ for the inner product on $L^{2}(\mu)$. With each Hankel form $\Gamma$ there is associated (by the Riesz-Lemma) a unique, anti-linear, continuous Hankel operator $H: \mathcal{H} L^{2}(\mu) \rightarrow \mathcal{H} L^{2}(\mu)$ satisfying

$$
\Gamma(f, g)=\langle f, H g\rangle=\int_{M} f \overline{H g} d \mu, \quad \forall f, g \in \mathcal{H} L^{2}(\mu) .
$$

When $\Gamma=\Gamma_{b}$ we write $H=H_{b}$, and call $b$ a symbol for $H$. Among the classical questions concerning bilinear forms such as (1.2) is the relation between properties of $b$ and continuity properties of $\Gamma_{b}$ as well as trace ideal properties of the associated operator $H_{b}$. These are some of the questions we will address in this paper.

Remark 1.1. The traditional concepts of Hankel matrices and Hankel operators on the Hardy space $[\mathbf{P a}, \mathbf{P o} \mathbf{1}, \mathbf{P o} \mathbf{2}, \mathbf{Z h}$ ] have been generalized in various directions $[\mathbf{P}, \mathbf{R o 1}, \mathbf{J P R}, \mathbf{J a 2}, \mathbf{H R}]$. Our notion of Hankel forms and (small) Hankel operators essentially coincides with the one in [JPR]; one only has to replace their domain $\Omega \subset \mathbb{C}^{d}$ by $M$. For the distinction between small and big Hankel operators see, e.g., $[\mathbf{J a 2}, \mathbf{Z h}]$, and for the choice of linear or anti-linear Hankel operators see $[\mathbf{P}, \mathbf{J P R}]$. Relations between Hankel operators and other fields are discussed in [Po1, PK, Pe2, Pe3, Pa].

In this paper we are going to assume that $M$ is endowed with a Hermitian metric $g$ in addition to a smooth probability measure $\mu$. There is naturally associated to $g$ and $\mu$ a Dirichlet form operator $\nabla^{*} \nabla$ on $C^{\infty}(M)$ which is defined by

$$
\left\langle\nabla^{*} \nabla f, \psi\right\rangle=\int_{M} g(\nabla f(z), \nabla \bar{\psi}(z)) d \mu(z), \quad f \in C^{\infty}(M), \psi \in C_{c}^{\infty}(M) .
$$

Moreover, there is always at least one nonnegative self-adjoint version of the differential operator $\nabla^{*} \nabla$ in $L^{2}(\mu)$. Denote by $A$ such a self-adjoint version. The central theme of this work is to relate properties of the Hankel operator $H_{b}$ to properties of the Dirichlet form operator $A$. In the classical

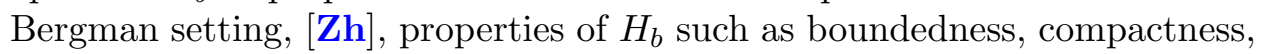
etc., are determined by properties of the derivative $b^{\prime}$. In our setting we will use the operator $A$ to define regularity of $b$. We will need to know that $A$ is "strong" enough to convert regularity into $L^{p}$ bounds. To this end we will assume, in the key theorems, that the quadratic form of $A$ satisfies a logarithmic Sobolev inequality, cf. (2.21), and we will exploit the resulting hypercontractivity of the semigroup $e^{-t A}$. 
In Section 4 we will give sufficient conditions on a symbol $b$ which ensure that the Hankel operator $H_{b}$ is respectively bounded or compact or in some Schatten class. The hypercontractivity of the semigroup $e^{-t A}$ in an appropriate scale of holomorphic function spaces will play a central role. We will give in Section 3 five classes of examples to which our theory is applicable in some form, including Gaussian and non-Gaussian measures on $\mathbb{C}^{d}$, the weighted Bergman spaces over the unit disk, a general class of measures on $\mathbb{C}-\{0\}$ and a class of measures on the Riemann surface for $z^{1 / n}$.

In $[\mathbf{J P R}]$ the authors asked, with a view toward extending their results to complex manifolds, whether there is a generalization for the algebra of polynomials in $\mathbb{C}^{d}$. It happens that when $(M, g)$ is complete and a logarithmic Sobolev inequality holds, the union of the finite spectral subspaces of $\left.A\right|_{\mathcal{H} L^{2}(\mu)}$ forms an algebra, $\mathcal{R}$, imitating well the algebra of polynomials in several complex variables. Even when $M$ is not complete there is sometimes an algebra $\mathcal{R}$ determined by $A$ in a similarly intrinsic manner. This is the case, for example, for the Riemann surface for $z^{1 / n}$. We will see in Sections 4 and 5 that the natural algebra $\mathcal{R}$ is a good analog for the polynomials on $\mathbb{C}^{d}$. This, in combination with the strong hypercontractivity of the semigroup $e^{-t A}$, offers a promising approach to the study of Hankel operators over complex manifolds. We will frequently compare our results to those in [JPR], which is the definitive work on extensions of the concept of Hankel operator to Gaussian spaces.

Section 5 presents some computable examples showing that some of the sufficient conditions in Section 4 are best possible of the given form and some are not. The algebra $\mathcal{R}$, which we will refer to as the spectral algebra, is especially useful for computing Hilbert-Schmidt norms. The triangular structure, $\left(h_{n+m}\right)$, of the classical Hankel forms shows up in this context. As we will see, the spectral algebra yields a natural orthogonal decomposition of any Hilbert-Schmidt Hankel operator $H_{b}$ into finite rank operators. This can sometimes be used to characterize completely those holomorphic symbols $b$ corresponding to Hilbert-Schmidt Hankel operators. We illustrate this by Gauss measure on $\mathbb{C}^{d}$, the weighted Bergman space, the space $\mathcal{H} L^{2}\left(\mathbb{C},\left(2 \pi a^{2}\right)^{-1} e^{-|z| / a} d x d y\right)$ and a space of holomorphic functions over the $n$-sheeted Riemann surface for $z^{1 / n}$. It will be shown how very far from necessary our sufficient conditions of Section 4 are when applied to the weighted Bergman spaces. It seems possible that our techniques will extend to these spaces with the help of some stronger form of hypercontractivity of the associated semigroup. See e.g., [GR]. But we have not explored this.

In Section 6 we will see that the reproducing kernel $K_{z}$ relates extremely well to the semigroup $e^{-t A}$. Under simple conditions on $A$ we will deduce strong regularity and size properties of $K_{z}$. 


\section{Preliminaries: Dirichlet forms in holomorphic function spaces.}

In this section we will establish notation and at the same time review some background that is not standard in the literature on Hankel operators. In particular the connection between a logarithmic Sobolev inequality for a Dirichlet form and hypercontractivity of the associated semigroup will be surveyed and some elementary calculus over complex manifolds will be reviewed.

We denote by $M$ an $n$ dimensional (real) manifold with Riemannian metric $g$. Let $\mu$ be a probability measure on $M$ which, in each coordinate chart $\left(U, x_{1}, \ldots, x_{n}\right)$ is given by a smooth density $\rho$ :

$$
d \mu(x)=\rho(x) d x_{1} \ldots d x_{n} \text { in } U .
$$

There is naturally associated to the triple $(M, g, \mu)$ the sesquilinear form

$$
Q_{0}(f, \psi)=\int_{M} g(\nabla f(x), \nabla \bar{\psi}(x)) d \mu(x) \quad f \in C^{\infty}(M), \quad \psi \in C_{c}^{\infty}(M) .
$$

This gives rise to a second order differential operator

$$
\nabla^{*} \nabla: C^{\infty}(M) \rightarrow C^{\infty}(M)
$$

which is defined by the equations

$$
\left\langle\nabla^{*} \nabla f, \psi\right\rangle_{L^{2}(\mu)}=Q_{0}(f, \psi) \quad f \in C^{\infty}(M), \quad \psi \in C_{c}^{\infty}(M) .
$$

In these equations the bilinear form $g$ on the tangent space $T_{x}(M)$ is, as usual, extended in a complex bilinear manner to the complexification $\mathbb{C} \otimes$ $T_{x}(M)$.

Example 2.1 (Gauss measure on $\mathbb{R}^{n}$ ). Let

$$
p_{a}(x)=(2 \pi a)^{-n / 2} e^{-|x|^{2} / 2 a} \quad x \in \mathbb{R}^{n}, \quad a>0 .
$$

Choose for $g$ the standard metric on $\mathbb{R}^{n}$. Then an integration by parts gives

$$
\begin{aligned}
Q_{0}(f, \psi) & =\int_{\mathbb{R}^{n}} \sum_{j=1}^{n}\left(\partial f / \partial x_{j}\right)\left(\partial \bar{\psi} / \partial x_{j}\right) p_{a}(x) d x \\
& =-\int \sum p_{a}(x)^{-1}\left[\left(\partial / \partial x_{j}\right)\left(p_{a}(x) \partial f / \partial x_{j}\right)\right] \bar{\psi}(x) p_{a}(x) d x
\end{aligned}
$$

for $f \in C^{\infty}\left(\mathbb{R}^{n}\right)$ and $\psi \in C_{c}^{\infty}\left(\mathbb{R}^{n}\right)$. Comparison with (2.3) yields

$$
\left(\nabla^{*} \nabla f\right)(x)=-\Delta f(x)+a^{-1}(x, \nabla f(x))_{\mathbb{R}^{n}}
$$

which is the Ornstein-Uhlenbeck operator over $\mathbb{R}^{n}$.

In general, in a local chart, cf. (2.1), one can compute $\nabla^{*} \nabla f$ explicitly by choosing $\psi \in C_{c}^{\infty}(M)$ with support in $U$. If one defines $g_{j k}(x)=$ $g\left(\partial / \partial x_{j}, \partial / \partial x_{k}\right)$ and writes $g^{j k}(x)$ for the entries of the inverse matrix then a straightforward integration by parts as in the Gaussian example gives 
$\nabla^{*} \nabla f(x)=-\rho(x)^{-1} \sum_{i, j=1}^{n}\left(\partial / \partial x_{j}\right)\left[\rho(x) g^{i j}(x) \partial f(x) / \partial x_{i}\right]$ for $x \in U$. We will not have occasion to use this explicit expression. But this expression makes clear that (2.3) always defines $\nabla^{*} \nabla$ as a second order differential operator on $C^{\infty}(M)$.

We will choose a self-adjoint version of $\nabla^{*} \nabla$ as follows. Denote by $Q$ the closed quadratic (Dirichlet) form in $L^{2}(\mu)$ with $C_{c}^{\infty}(M)$ as a core and which is given by

$$
Q(f)=\int_{M} g(\nabla f(z), \nabla \bar{f}(z)) d \mu(z), \quad f \in C_{c}^{\infty}(M)
$$

There is a unique, nonnegative self-adjoint operator $A$ in $L^{2}(\mu)$ such that the domain of $Q, D(Q)$, is exactly the domain of $A^{\frac{1}{2}}$ and

$$
Q(f)=\left\|A^{\frac{1}{2}} f\right\|_{2}^{2}, \quad \forall f \in D(Q) .
$$

Here and in the following $\|\cdot\|_{p}$ denotes the norm in $L^{p}(\mu)$. Dirichlet forms over manifolds were investigated e.g., in $[\mathbf{A H S}]$ and $[\mathbf{F u 1}]$. See e.g., $[\mathbf{K a}]$ and [Da1] for general background on Dirichlet forms and their associated self-adjoint operators.

Comparing (2.3) with the polarized version of (2.7) one sees that $\left\langle\nabla^{*} \nabla f\right.$, $\psi\rangle=\langle A f, \psi\rangle$ for $f$ and $\psi$ in $C_{c}^{\infty}(M)$. So $A f=\nabla^{*} \nabla f$ for $f$ in $C_{c}^{\infty}(M)$. $A$ is thus a self-adjoint extension of $\nabla^{*} \nabla \mid C_{c}^{\infty}(M)$.

If $M$ is not complete then the choice of $C_{c}^{\infty}(M)$ as a core for $Q$ corresponds to the choice of Dirichlet boundary conditions for $A$. We will limit our discussions here to this case. But the use of non-Dirichlet boundary conditions is needed for the weighted Bergman spaces. This is discussed in Examples 3.11 and 3.12 and in more detail in [G4].

By the spectral theorem the operators $e^{-t A}$ are contraction operators in $L^{2}(\mu)$ for $t \geq 0$ because $A$ is nonnegative. But in addition, because $A$ is obtained from a Dirichlet form, the operators $e^{-t A}$ are also contractions in $L^{p}$ for all $p \in[1, \infty]$. I.e., $\left\|e^{-t A} f\right\|_{p} \leq\|f\|_{p}$ for $f \in L^{p} \cap L^{2}$. Among the many good books [BH, Da1, Da2, Fu1, Fu2, Ka, MR, RS] discussing the relation between a Dirichlet form and the associated semigroup the expositions in [Da1, Da2, Fu1] are closest to our needs.

Suppose now that $M$ is actually a complex manifold and that the metric $g$ is Hermitian. (See below for a discussion of this property.) Although the space $\mathcal{H} L^{2}$ of holomorphic $L^{2}$ functions is always a closed subspace of $L^{2}$, the triple $(M, g, \mu)$ picks out a (possibly) smaller closed subspace of $\mathcal{H} L^{2}$ as 
follows. Let $\mathcal{H}$ denote the space of all holomorphic functions on $M$. Define

$$
\begin{aligned}
\mathcal{H}^{2} & :=\text { closure of } \mathcal{H} \cap D(Q) \text { in } L^{2}(\mu), \\
\mathcal{H}^{p} & :=\mathcal{H}^{2} \cap L^{p} \text { for } 2<p<\infty, \\
\mathcal{H}^{p} & :=\text { closure of } \mathcal{H}^{2} \text { in } L^{p}(\mu) \text { for } 0<p<2 .
\end{aligned}
$$

Here and elsewhere, for $0<p<1$, we use the metric $d(f, g)=\|f-g\|_{p}^{p}$ on $L^{p}$ to define closure.

Very often one has simply $\mathcal{H}^{2}=\mathcal{H} L^{2}$. But a very interesting deviation from this occurs when $M$ is the $n$ sheeted Riemann surface for $z^{1 / n}$ (cf. Example 3.4). Most of our results in holomorphic function spaces take place in the spaces $\mathcal{H}^{p}$. We will need to know that the operators $e^{-t A}$ leave these spaces invariant. To this end we will need to impose some further conditions on the triple $(M, g, \mu)$. Let us return to the sesquilinear form $Q_{0}$ given in (2.2) and recompute $\nabla^{*} \nabla f$ in complex coordinates when $f$ is a holomorphic function. We will show that $\nabla^{*} \nabla: \mathcal{H} \rightarrow C^{\infty}(M)$ reduces to a first order differential operator as a consequence of the Cauchy-Riemann equations.

Let $z_{j}=x_{j}+i y_{j}$, for $j=1, \ldots, m$ be complex coordinates in an open set $U$ of $M$. So $M$ has real dimension $2 m$. For each point $x \in M$ the tangent space $T_{x}(M)$ is spanned by the basis $\left\{\partial / \partial x_{j}, \partial / \partial y_{j}\right\}_{j=1}^{m}$. Define a linear operator $J: T_{x} \rightarrow T_{x}$ by $J \partial / \partial x_{j}=\partial / \partial y_{j}$ and $J \partial / \partial y_{j}=-\partial / \partial x_{j}, j=1, \ldots, m$. (It is simple to check that $J$ does not depend on the choice of complex coordinates at $x$.) By definition the Riemannian metric $g$ is Hermitian if $g_{x}(J v, J w)=g_{x}(v, w)$ for all $v, w \in T_{x}$ and all $x \in U$. For example if $M=\mathbb{C}$ with complex coordinate $z=x+i y$ then to say that $g$ is Hermitian means that $g_{z}(\cdot, \cdot)$ is invariant under 90 degree rotations in the tangent plane at $z$ and consequently is just a ( $z$ dependent) multiple of the standard inner product on $\mathbb{R}^{2}$.

It is usually simpler to make computations in terms of the dual metric $h_{x}(\cdot, \cdot)$ on $T_{x}^{*}(M)$. It is straighforward to verify that the adjoint $J^{\prime}$ on $T_{x}^{*}$ satisfies $J^{\prime} d x_{j}=-d y_{j}$ and $J^{\prime} d y_{j}=d x_{j}$ for all $j$. Moreover the invariance of $g$ under $J$ reflects itself in the invariance of $h$ under $J^{\prime}: h_{x}\left(J^{\prime} \alpha, J^{\prime} \beta\right)=$ $h_{x}(\alpha, \beta)$ for all $\alpha$ and $\beta$ in $T_{x}^{*}$. The reader can easily deduce from these identities that $h_{x}\left(d x_{j}, d x_{k}\right)=h_{x}\left(d y_{j}, d y_{k}\right)$ and that $h_{x}\left(d x_{j}, d y_{k}\right)+h_{x}\left(d y_{j}, d x_{k}\right)=$ 0 . Passing now to the complexified cotangent space $\mathbb{C} \otimes T_{x}^{*}$, we put, as is customary, $d z_{j}=d x_{j}+i d y_{j}$ and $d \bar{z}_{j}=d x_{j}-i d y_{j}$ and we also extend $h_{x}$ complex bilinearly (not sesquilinearly). The last identities then yield

$$
h_{x}\left(d z_{j}, d z_{k}\right)=h_{x}\left(d \bar{z}_{j}, d \bar{z}_{k}\right)=0, \quad j, k=1, \ldots, m
$$

and $h_{x}\left(d z_{j}, d \bar{z}_{j}\right)=2 h_{x}\left(d x_{j}, d x_{j}\right)=2 h_{x}\left(d y_{j}, d y_{j}\right)$.

Now $g(\nabla f, \nabla \bar{\psi})=h(d f, d \bar{\psi})$ where $d f(x)$ and $d \bar{\psi}(x)$ are to be interpreted as elements of $\mathbb{C} \otimes T_{x}^{*}(M)$. Defining, as is customary, $\partial / \partial z_{j}=(1 / 2)\left(\partial / \partial x_{j}-\right.$ $\left.i \partial / \partial y_{j}\right)$ and $\partial / \partial \bar{z}_{j}=(1 / 2)\left(\partial / \partial x_{j}+i \partial / \partial y_{j}\right)$ (interpreted as elements of 
$\mathbb{C} \otimes T_{x}(M)$ for each point $x$ in the chart $\left.U\right)$, one can verify easily that in $U$ one has

$$
d f=\sum_{j=1}^{m}\left[\left(\partial f / \partial x_{j}\right) d x_{j}+\left(\partial f / \partial y_{j}\right) d y_{j}\right]=\sum_{j=1}^{m}\left[\left(\partial f / \partial z_{j}\right) d z_{j}+\left(\partial f / \partial \bar{z}_{j}\right) d \bar{z}_{j}\right]
$$

for any function $f$ in $C^{\infty}(M)$. If $f$ is holomorphic in $U$ then $\partial f / \partial \bar{z}_{j}=0$ for $j=1, \ldots, m$ by the Cauchy-Riemann equations. Thus if $f \in \mathcal{H}$ and $\psi \in C_{c}^{\infty}(M)$ with support in $U$ then one has simply

$$
h(d f, d \bar{\psi})=h\left(\sum_{j=1}^{m}\left(\partial f / \partial z_{j}\right) d z_{j}, \sum_{k=1}^{m}\left(\partial \bar{\psi} / \partial \bar{z}_{k}\right) d \bar{z}_{k}\right) \text { in } U,
$$

all other terms being zero by (2.11). The identity (2.12) allows one to make efficient use of the fact that $f$ is holomorphic. Let $h_{j k}(x)=h_{x}\left(d z_{j}, d \bar{z}_{k}\right)$. Then, if $f$ is in $\mathcal{H}$ and $\psi \in C_{c}^{\infty}(M)$ with support in $U,(2.12)$ and an integration by parts gives

$$
\begin{aligned}
Q_{0}(f, \psi) & =\sum_{j, k} \int_{x(U)} h_{j k}(x)\left(\partial f / \partial z_{j}\right)\left(\partial \bar{\psi} / \partial \bar{z}_{k}\right) \rho(x) d x \\
& =-\sum_{j, k} \int_{x(U)}\left[\partial / \partial \bar{z}_{k}\left(h_{j k}(x) \rho(x) \partial f / \partial z_{j}\right)\right] \bar{\psi}(x) d x .
\end{aligned}
$$

Since $\left(\partial / \partial \bar{z}_{k}\right)\left(\partial f / \partial z_{j}\right)=0$, Equations (2.3) and (2.13) show that

$$
\nabla^{*} \nabla f=-\sum_{j, k} \rho(x)^{-1}\left[\left(\partial / \partial \bar{z}_{k}\right)\left\{h_{j k}(x) \rho(x)\right\}\right]\left(\partial f / \partial z_{j}\right) \quad \text { for } f \in \mathcal{H} .
$$

Although the right side of (2.14) is coordinate dependent the left side is not. Consequently one can assert that there is a complex vector field $Z$ on $M$ such that for any function $f \in C^{\infty}$

$$
\nabla^{*} \nabla f=Z f
$$

in any open set in which $f$ is holomorphic. Moreover $Z$ is unique under the condition that it be of type $(1,0)$ (i.e., a linear combination of the $\partial / \partial z_{j}$ only). In our local chart the coefficients of $\partial / \partial z_{j}$ can be read off from (2.14). Thus (2.15) defines a unique complex vector field of type $(1,0)$.

The following definition and properties are taken from $[\mathbf{G} 3, \mathbf{G} 4]$ to which we refer the reader for further details.

Definition 2.2. $(M, g, \mu)$ is holomorphic if for any function $f \in C^{\infty}(M)$ $\nabla^{*} \nabla f$ is holomorphic in any open set in which $f$ is holomorphic. In this case we also say that $\nabla^{*} \nabla$ is holomorphic.

A complex vector field $Z$ of type $(1,0)$ is called holomorphic if in each coordinate chart $Z=\sum_{j=1}^{m} a_{j}(z) \partial / \partial z_{j}$ for some holomorphic functions $a_{j}(z)$. One can see from (2.14) that $(M, g, \mu)$ is holomorphic if and only if the 
coefficient of $\partial f / \partial z_{j}$ is holomorphic for each $j$ and for every chart. Thus $(M, g, \mu)$ is holomorphic if and only if the vector field $Z$ of type $(1,0)$ defined by (2.15), (equivalently by (2.14) in local charts), is holomorphic.

Any complex vector field $Z$ on $M$ can be written in the form

$$
Z=\frac{1}{2}(X-i Y)
$$

for some unique real vector fields $X$ and $Y$. Henceforth, defining $Z$ to be the unique vector field of type $(1,0)$ given by $(2.15)$, we will always assume that the flow of the vector field $Y$, determined by (2.16), exists for all time, i.e., that $Y$ is complete. We will also assume that the flow of $Y$ preserves the metric $g$, i.e., that $Y$ is Killing. This holds automatically when $Z$ is holomorphic and $(M, g)$ is Kählerian (cf. [G3, Theorem 2.10]). In all of our examples the flow $e^{-t X}$ will exist for all $t \geq 0$. In that case we say that $X$ is one sided complete.

The following terminology summarizes the conditions on $(M, g, \mu)$ which will be needed almost everywhere in this paper.

Notation 2.3 (Standard Conditions). In the following we will say that $(M, g, \mu)$ satisfies standard conditions if $\nabla^{*} \nabla$ is holomorphic, $Y$ is complete and Killing and $X$ is one sided complete. Standard Conditions will be assumed to hold throughout this paper.

If the flow $e^{-t X}$ also exists for all real $t$ we will say that $X$ is two sided complete. This will occasionally be listed as an explicit assumption. Standard Conditions hold in all of our examples. But two sided completeness only holds in some of them.

Example 2.4 (Gauss measure on $\mathbb{C}^{m}$ ). Choose $a>0$ and let $\gamma_{a}$ be the Gauss measure on $\mathbb{C}^{m} \equiv \mathbb{R}^{2 m}$ given by the density (2.4). Thus $p_{a}(z)=$ $(2 \pi a)^{-m} e^{-|z|^{2} / 2 a}$. Let $g$ be the standard metric again on $\mathbb{R}^{2 m}$. Then we have $h_{j k}(x)=h\left(d z_{j}, d \bar{z}_{k}\right)=2 \delta_{j k}$ in Equation $(2.14)$. Since $p_{a}(z)^{-1}\left(\partial / \partial \bar{z}_{j}\right) p_{a}(z)=$ $-(2 a)^{-1} z_{j}$ we see that $(2.15)$ holds with

$$
Z f(z)=a^{-1} \sum_{j=1}^{m} z_{j} \partial f / \partial z_{j}, \quad f \in \mathcal{H} .
$$

Since the coefficient of $\partial f / \partial z_{j}$ is holomorphic $Z$ is a holomorphic vector field. To reiterate: $Z=a^{-1} \sum_{j=1}^{m} z_{j} \partial / \partial z_{j}$. So $\nabla^{*} \nabla$ is holomorphic for Gauss measure on $\mathbb{C}^{m}$ and the standard metric. The vector fields $X$ and $Y$ can be computed directly from (2.17). Using (2.16) and the identity $(x+i y)(1 / 2)(\partial / \partial x-i \partial / \partial y)=(1 / 2)(x \partial / \partial x+y \partial / \partial y)+(i / 2)(y \partial / \partial x-x \partial / \partial y)$ 
we see that

$(2.18)$

$X=a^{-1} \sum_{j=1}^{m}\left(x_{j} \partial / \partial x_{j}+y_{j} \partial / \partial y_{j}\right) \quad$ and $\quad Y=a^{-1} \sum_{j=1}^{m}\left(x_{j} \partial / \partial y_{j}-y_{j} \partial / \partial x_{j}\right)$.

$X$ is clearly the vector field which generates dilations: $e^{-t X} z=e^{-t / a} z$ for $z \in \mathbb{C}^{m}$ and all $t \in \mathbb{R}$. $Y$ generates rotations: $e^{-s Y} z=e^{-i s / a} z$. Since rotations preserve the standard metric $Y$ is Killing. $Y$ is clearly complete and $X$ is two sided complete. Thus Gauss measure together with the standard metric satisfies the Standard Conditions of Notation 2.3. Finally we note for later use that the logarithmic Sobolev inequality (2.21) below is also satisfied with $c=a$ and $\beta=0$. For a proof see e.g., [G1] or [G2].

The distinction between one sided completeness of $X$ and two sided completeness can be understood by contrasting the previous example with the weighted Bergman spaces over the unit disc (Example 3.12). We will see that the vector field $Z$ for the weighted Bergman spaces have the same form as in (2.17), $Z=a^{-1} z d / d z$, for $|z|<1$. Consequently $e^{-t X} x=e^{-t / a} z$, just as in the Gaussian case. But for a finite negative time $e^{-t / a} z$ leaves the unit disc if $z \neq 0$. Therefore $X$ is only one sided complete for these spaces.

Remark 2.5 (Relation of $\mathcal{H}^{p}$ to $\mathcal{H} L^{p}$ ). If $\nabla^{*} \nabla$ is holomorphic, $Y$ is complete and Killing, and $(M, g)$ is complete then $\mathcal{H}^{2}=\mathcal{H} L^{2}(\mu)$ by Theorem 2.14 of [G3]. Consequently $\mathcal{H}^{p}=\mathcal{H} L^{p}$ for $2 \leq p<\infty$ by (2.9). If $(M, g)$ is not complete then it can happen that $\mathcal{H}^{2}$ is properly contained in $\mathcal{H} L^{2}(\mu)$. This is the case for example if $M$ is the double sheeted Riemann surface for $\sqrt{z}, g$ is the standard metric and $\mu$ is the Gauss measure on $\mathbb{C}$ pulled up to $M$ with half the density on each sheet. In this example $\mathcal{H}^{2}$ is of codimension one in $\mathcal{H} L^{2}(\mu)$. The discrepancy between $\mathcal{H}^{2}$ and $\mathcal{H} L^{2}(\mu)$ reflects the singularity of $M$ at the origin. It is important to note that all the requirements on $(M, g, \mu)$ that we will need in this paper are satisfied in this example. So the event $\mathcal{H}^{2} \neq \mathcal{H} L^{2}$ should not be regarded as pathology. See Examples 3.4 and 3.5 for further details. In the Gaussian case (Example 2.4) the spaces $\mathcal{H}^{p}$ can be identified explicitly. One has $\mathcal{H}^{p}=\mathcal{H} L^{p}$ for $0<p<\infty$. See [G3, p. 197] for a proof.

Remark 2.6 (Multiplicativity of $\left.e^{-t A}\right)$. If the triple $(M, g, \mu)$ satisfies the Standard Conditions introduced in Notation 2.3 then the semigroup $e^{-t A}$ leaves each space $\mathcal{H}^{p}$ invariant as does also the group of operators $e^{i s A}$ for $s$ real, [G3, Theorem 2.11]. Equation (2.15) shows that $\nabla^{*} \nabla$ reduces to a first order differential operator on $\mathcal{H}$. This is reflected in the following identity for the semigroups generated by $A$ and $i A$.

$$
\left(e^{-(t+i s) A} f\right)(z)=f\left(e^{-t X} e^{-s Y} z\right) \quad \text { for } f \in \mathcal{H}^{p}, t \geq 0, s \in \mathbb{R}, p \in(0, \infty) .
$$


(See [G3] for proofs. Although (2.19) is stated in [G3] for $f \in \mathcal{H}^{2}$ it follows for $f \in \mathcal{H}^{p}$ by restriction, for $p>2$ and by continuity, for $0<p<2$.) Of particular significance for us will be the identity (2.19) when $s=0$. If $f, h$, and $f h$ are in $\mathcal{H}^{2}$ then (2.19) yields the multiplicative identity

$$
e^{-t A}(f h)=\left(e^{-t A} f\right)\left(e^{-t A} h\right) \text { for all } t \geq 0,
$$

which lies at the heart of our technique for studying Hankel operators. In addition, under our Standard Conditions, the group $e^{i s A}$ is isometric in all of the spaces $\mathcal{H}^{p}$, for $0<p<\infty$, while the semigroup $e^{-t A}$ is a contraction in all of these spaces.

Notation 2.7 (Logarithmic Sobolev inequality). As already noted in the Introduction, we will define regularity of a Hankel symbol $b$ with the help of the operator $A$. In order for regularity to relate well to $L^{p}$ properties of functions we will assume, in some of our theorems on boundedness of Hankel operators, that $A$ is "strong" in the sense that its quadratic form $Q$ satisfies the following logarithmic Sobolev inequality.

$$
\int_{M}|f(z)|^{2} \log |f(z)| d \mu \leq c Q(f)+\beta\|f\|_{2}^{2}+\|f\|_{2}^{2} \log \|f\|_{2}, \forall f \in D(Q)
$$

for some $c>0$ and $\beta \geq 0$.

Our proof of the boundedness of Hankel operators will be based on the following strong hypercontractivity inequalities. A proof of the following theorem may be found in [G3].

Theorem 2.8 (Strong hypercontractivity, [G3]). If, in addition to the Standard Conditions of Notation 2.3, the logarithmic Sobolev inequality (2.21) holds then, for $0<p \leq q<\infty$,

$$
\left\|e^{-t A} f\right\|_{\mathcal{H}^{q}} \leq M(p, q)\|f\|_{\mathcal{H}^{p}} \quad \text { for } f \in \mathcal{H}^{p} \text { and } t \geq t_{J}(p, q),
$$

where

$$
t_{J}(p, q)=\frac{c}{2} \log \left(\frac{q}{p}\right)
$$

and

$$
M(p, q)=\exp \left[2 \beta\left(p^{-1}-q^{-1}\right)\right] .
$$

Notation 2.9 (The Spectral Algebra). Assume that Standard Conditions hold. Consider the restriction of $A$ to the invariant subspace $\mathcal{H}^{2}$. For $a>0$ let $\mathcal{R}_{a}$ be the spectral subspace of $\left.A\right|_{\mathcal{H}^{2}}$ for the interval [0,a]. Define

$$
\mathcal{R}:=\bigcup_{0<a<\infty} \mathcal{R}_{a}
$$

By the spectral theorem $\mathcal{R}$ is dense in $\mathcal{H}^{2}$. It often happens that $\mathcal{R}$ is closed under pointwise multiplication and is therefore an algebra in the pointwise 
product. In this case we will refer to $\mathcal{R}$ as the spectral algebra of $(M, g, \mu)$. Clearly this algebra is functorially associated to the triple $(M, g, \mu)$ when Standard Conditions hold.

There are circumstances in which $\mathcal{R}$ is automatically closed under pointwise multiplication. For example when the logarithmic Sobolev inequality (2.21) holds and $\mathcal{H}^{2}=\mathcal{H} L^{2}$ then $\mathcal{R}$ is an algebra, [G3, Theorem 2.17]. Moreover $\mathcal{H}^{2}=\mathcal{H} L^{2}$ whenever $(M, g)$ is complete, [G3, Theorem 2.14]. Even if $\mathcal{H}^{2} \neq \mathcal{H} L^{2}(\mu)$ it can happen that $\mathcal{R}$ is closed under multiplication. This is the case for the Riemann surface of Example 3.4. In fact we have no example in which the logarithmic Sobolev inequality (2.21) holds and $\mathcal{R}$ is not an algebra. In the classical case of Gauss measure on $\mathbb{C}^{d}$ the algebra $\mathcal{R}$ consists exactly of the holomorphic polynomials. The only examples known to us in which $\mathcal{R}$ fails to be closed under multiplication are those, such as Example 3.18 below, for which $\mu$ fails to have finite moments of all orders.

All of our results on Hankel operators, i.e., Sections 4 and 5, will require that $\mathcal{R}$ be an algebra in the pointwise product. The reason for this is that we will have to use the identity (2.20) in a fundamental way in the proof of our main theorem, Theorem 4.2. So we will need to know that $f h \in \mathcal{H}^{2}$ when $f$ and $h$ are in some dense subspace of $\mathcal{H}^{2}$ (such as $\mathcal{R}$ ). The structure of the spectral algebra will be described in Section 5 .

Overview 2.10. All results in this paper will depend on one or more of the following assumptions:

(a) Standard Conditions hold. (See Notation 2.3.) This will be assumed throughout the paper.

(b) $\mathcal{R}$ is an algebra under pointwise multiplication. (See Notation 2.9.) This will be assumed whenever we are dealing with Hankel operators. For example it is not needed in Section 6 where we discuss only the reproducing kernel.

(c) The logarithmic Sobolev inequality (2.21) holds. This will be assumed when necessary.

\section{Examples.}

This section is devoted to describing examples. All the examples in this section and paper will satisfy Standard Conditions. All will have the property that $\mathcal{R}$ is an algebra, with the exception of the pathological Example 3.18. The logarithmic Sobolev inequality (2.21) will be proven in some of the examples. Section 4, which contains the main results of this paper, may be read independently of this section.

Example 3.1 (Non-Gaussian measures on $\mathbb{C}^{m}$ ). We will construct a class of non-Gaussian examples on $\mathbb{C}^{m}$ which satisfy our standard conditions as well as the logarithmic Sobolev inequality (2.21) and for which $\mathcal{R}$ is an 
algebra. In order for a triple $(M, g, \mu)$ to be holomorphic the metric $g$ and measure $\mu$ must be related in such a way that the vector field $Z$ in Equation (2.15) is holomorphic. This imposes a tight relation between $g$ and $\mu$. If $M=\mathbb{C}^{m}$ and $\mu$ is not simply a Gaussian measure then the metric $g$ must deviate from the standard one. In [G3, Section 5] a class of non-Gaussian measures $\mu$ and corresponding metrics $g$ is described such that $\left(\mathbb{C}^{m}, g, \mu\right)$ satisfies our standard conditions, $X$ is two-sided complete, (2.21) holds and $\mathcal{R}$ is an algebra. We will describe this class of examples here and at the same time extend the class. All of the theorems of this paper will be applicable to these classes of examples (with the likely exception of Corollary 4.18, which deals with Hankel operators into $\mathcal{H}^{q}, q \neq 2$ ).

Let $M=\mathbb{C}^{m}$. Denote by $x_{1}, \ldots, x_{2 m}$ the standard linear coordinates on $\mathbb{C}^{m} \equiv \mathbb{R}^{2 m}$ with $z_{k}=x_{2 k-1}+i x_{2 k}, k=1,2, \ldots, m$. Let $\sigma$ be a strictly positive function in $C^{\infty}\left(\mathbb{R}^{2 m}\right)$. Define a metric on $\mathbb{R}^{2 m}$ by

$$
g_{x}\left(\partial / \partial x_{j}, \partial / \partial x_{k}\right)=\delta_{j k} / \sigma(x) .
$$

Then $g$ is a Hermitian metric as one can see from the definition preceding (2.11). The dual metric is

$$
h_{x}\left(d x_{j}, d x_{k}\right)=\delta_{j k} \sigma(x) \quad j, k=1, \ldots, 2 m .
$$

$g$ extends complex bilinearly to $\mathbb{C} \otimes_{\mathbb{R}} T\left(\mathbb{R}^{2 m}\right)$ and the complex bilinear extension of the dual metric satisfies

$$
h_{x}\left(d z_{r}, d \bar{z}_{s}\right)=2 \delta_{r s} \sigma(x) .
$$

We will take for $\mu$ a probability measure on $\mathbb{R}^{2 m}$ with an infinitely differentiable positive density. Thus we put

$$
d \mu(x)=\rho(x) d x,
$$

where $d x=d x_{1} \ldots d x_{2 m}$ and $\rho$ is a strictly positive function in $C^{\infty}\left(\mathbb{R}^{2 m}\right)$ with integral equal to one. It will be convenient to express the Dirichlet form for $\left(\mathbb{C}^{m}, g, \mu\right)$ in terms of $\rho$ and $w$, where

$$
w(x)=\sigma(x) \rho(x) \quad x \in \mathbb{R}^{2 m} .
$$

The local coordinates used in (2.14) may now be taken to be the global coordinates on $\mathbb{R}^{2 m}$ (with $x_{2 k-1}$ replaced by $x_{k}$ and $x_{2 k}$ replaced by $y_{k}$ ). Then (2.13) combined with (3.2) and (3.4) yields

$$
Q_{0}(f, \psi)=2 \int_{\mathbb{C}^{m}} \sum_{j=1}^{k}\left(\partial f / \partial z_{j}\right)\left(\partial \bar{\psi} / \partial \bar{z}_{j}\right) w(x) d x .
$$

Similarly, (2.14) combined with (3.2) and (3.4) yields

$$
\left(\nabla^{*} \nabla f\right)(z)=-2 \sum_{j=1}^{m} \rho(x)^{-1}\left(\partial w / \partial \bar{z}_{j}\right) \partial f / \partial z_{j}, \quad f \in \mathcal{H}\left(\mathbb{C}^{m}\right) .
$$


Inserting $f(z)=z_{k}$ one sees that $\nabla^{*} \nabla$ is holomorphic if and only if each coefficient

$$
\rho(x)^{-1} \partial w / \partial \bar{z}_{k} \quad \text { is holomorphic for } \quad k=1, \ldots, m .
$$

Here is a large class of functions $w$ and $\rho$ satisfying (3.6). Suppose that $\varphi$ is a strictly positive function in $C^{\infty}([0, \infty))$. Assume further that its derivative $\varphi^{\prime}$ satisfies $\varphi^{\prime}(s)<0$ for $0 \leq s<\infty$. Define

$$
\begin{gathered}
w(z)=\varphi\left(|z|^{2}\right), \\
\rho(z)=-b \varphi^{\prime}\left(|z|^{2}\right), \quad b=\text { constant }>0, \\
\sigma(z)=w(z) / \rho(z) .
\end{gathered}
$$

Then $\partial w / \partial \bar{z}_{k}=z_{k} \varphi^{\prime}\left(|z|^{2}\right)$. So

$$
\rho(z)^{-1} \partial w / \partial \bar{z}_{k}=-b^{-1} z_{k} .
$$

Thus (3.6) holds and

$$
\left(\nabla^{*} \nabla f\right)(z)=2 b^{-1} \sum_{k=1}^{m} z_{k} \partial f / \partial z_{k} \quad f \in \mathcal{H}\left(\mathbb{C}^{m}\right) .
$$

Hence $\nabla^{*} \nabla$ is holomorphic for $\left(\mathbb{C}^{m}, \sigma, \rho\right)$, wherein we have written $\sigma, \rho$ to signify the metric (3.1) and the measure (3.3).

The vector fields $Z, X$ and $Y$ (cf. (2.15), (2.16)) are therefore given by

$$
Z=2 b^{-1} \sum_{k=1}^{m} z_{k} \partial / \partial z_{k}
$$

$X=2 b^{-1} \sum_{k=1}^{m}\left(x_{k} \partial / \partial x_{k}+y_{k} \partial / \partial y_{k}\right)$ and $Y=2 b^{-1} \sum_{k=1}^{m}\left(x_{k} \partial / \partial y_{k}-y_{k} \partial / \partial x_{k}\right)$.

So $\exp (-t X) z=e^{-2 t / b} z, \exp (-s Y) z=e^{-2 i s / b} z$ and $\exp (-t X-s Y) z=$ $e^{-2(t+i s) / b} z$ for $z \in \mathbb{C}^{m}, s, t \in \mathbb{R}$. Both vector fields $X$ and $Y$ are complete, as is also the sum, $t X+s Y$. Moreover $Y$ is Killing because $\sigma(z)$ depends only on $|z|^{2}$, which is invariant under the rotations $\exp (-s Y)$. The constant $b$ may be chosen to normalize $\rho$ when $\rho$ is integrable. Thus the class of examples parametrized by a function $\varphi$ as in (3.7)-(3.9) all satisfy our standard conditions. But we will restrict $\varphi$ further so as to ensure that (2.21) holds.

In this large class of examples the operator $\nabla^{*} \nabla$, as an operator on $\mathcal{H}\left(\mathbb{C}^{m}\right)$, is the same in all cases in spite of the fact that the Hilbert spaces $\mathcal{H} \cap L^{2}\left(\mathbb{C}^{m}, \rho\right)$ (respectively $\left.\mathcal{H}^{2}\right)$ may be quite different. The self-adjoint versions, $A$, of $\nabla^{*} \nabla$ as operators in $\mathcal{H} \cap L^{2}\left(\mathbb{C}^{m}, \rho\right)$ (respectively $\mathcal{H}^{2}$ ) may not be unitarily equivalent for different $\rho$. For example $\mathcal{H}^{2}$ may be infinite dimensional, as in the Gaussian case above, or finite dimensional, as in Example 3.18 below. 
The Gaussian case, Example 2.4, may be obtained as a special case of this class of examples by choosing $\varphi(s)=(2 \pi a)^{-m} e^{-s /(2 a)}$ and $b=2 a$. In this case (3.9) gives $\sigma \equiv 1$. So $g$ is indeed the standard metric, as in Example 2.4.

We will construct some non-Gaussian examples satisfying both the Standard Conditions and the logarithmic Sobolev inequality (2.21) by perturbing the Gaussian case. Take

$$
\varphi(s)=(2 \pi)^{-m} e^{-(s+v(s)) / 2}
$$

in (3.7) and (3.8) with $v$ in $C^{\infty}([0, \infty))$. Assume that $1+v^{\prime}(s)>0$. Now $\varphi^{\prime}(s)=-(1 / 2)\left(1+v^{\prime}(s)\right) \varphi(s)<0$. So $w(z)=(2 \pi)^{-m} e^{-\left(|z|^{2}+v\left(|z|^{2}\right)\right) / 2}$ and $\rho(z)=(b / 2)(2 \pi)^{-m}\left(1+v^{\prime}\left(|z|^{2}\right)\right) e^{-\left(|z|^{2}+v\left(|z|^{2}\right)\right) / 2}$ are both positive. Also $\sigma(z)=2 b^{-1}\left(1+v^{\prime}\left(|z|^{2}\right)\right)^{-1}>0$. So $\sigma$ and $\rho$ determine a holomorphic Dirichlet form as in (3.1) and (3.3) when $\rho$ is integrable. It was shown in [G3, p. 199] that if:

(a) $v$ is bounded, and

(b) $0<\alpha_{1} \leq 1+v^{\prime}(s) \leq \alpha_{2}<\infty$ for some constants $\alpha_{1}$ and $\alpha_{2}$ and all $s$, then:

(i) (2.21) holds with $\beta=0$,

(ii) $\left(\mathbb{C}^{m}, g\right)$ is complete,

(iii) $\mathcal{R}$ is an algebra, namely the set of all holomorphic polynomials on $\mathbb{C}^{m}$, (iv) $\mathcal{H}^{p}=\mathcal{H} \cap L^{p}, \quad 0<p<\infty$.

The proof of (2.21) was based on the perturbation theorem of Holley and Stroock [HS]. However it is of interest to allow $v$ to be unbounded (cf. Remark 3.3). To this end we will apply the perturbation theorem of Aida and Shigekawa $[\mathbf{A S}]$ and replace the conditions (a) and (b) as follows.

Theorem 3.2. (Logarithmic Sobolev inequality for non-Gaussian measures on $\left.\mathbb{C}^{m}\right)$. Assume that:

(c) $1+v^{\prime}(s)>0$ for $0 \leq s<\infty$, and

(d) $v^{\prime}(s) \rightarrow 0$ as $s \rightarrow \infty$.

Then (i), (ii) and (iii) hold. Moreover (iv) holds for $1 \leq p<\infty$.

Proof. Together, (c) and (d) imply that (b) holds. Condition (d) also implies that $s+v(s) \geq s / 2$ for large $s$. So $\rho$ is integrable and the constant $b$ may be chosen to normalize $\rho$. The inequalities (b) ensure that $\sigma$ is bounded and bounded away from zero. So the metric $g_{i j}(x)=\delta_{i j} / \sigma(x)$ is equivalent to the standard metric and $\left(\mathbb{R}^{2 m}, g\right)$ is therefore complete. Because of the equivalence of metrics it suffices to prove that (2.21) holds for the standard metric and the measure $\rho d x$. Now the factor $\left(1+v^{\prime}\left(|z|^{2}\right)\right)$ in $\rho(z)$ may be ignored by the Holley-Stroock Lemma [HS] because this factor is bounded and bounded away from zero by (b). Thus it suffices to prove (2.21) holds for the measure $\kappa e^{F(z)} d \gamma_{1}(z)$ where $F(z)=-v\left(|z|^{2}\right) / 2$ and $\kappa$ is a normalization 
constant. To this end we may apply the perturbation theorem of Aida and Shigekawa [AS, Theorem 3.4]. For this we need only verify that

$$
\int_{\mathbb{R}^{2 m}} \exp \left(p|\nabla F(x)|^{2}\right) d \gamma_{1}(x)<\infty
$$

for all $p<\infty$. But $|\nabla F(x)|^{2}=\left|v^{\prime}\left(|x|^{2}\right) x\right|^{2}=v^{\prime}\left(|x|^{2}\right)^{2}|x|^{2}$ for $x \in \mathbb{R}^{2 m}$. Condition (d) now shows that, for any $p<\infty, p|\nabla F(x)|^{2} \leq|x|^{2} / 4$ for all sufficiently large $|x|$. (3.15) now follows. Since all polynomials are in $L^{2}\left(\mathbb{C}^{m}, \rho(x) d x\right)$ we have $\mathcal{R}=\{$ holomorphic polynomials $\}$ by [G3, Theorem 5.5]. In particular $\mathcal{R}$ is an algebra. Item (iv) also follows from [G3, Theorem 5.5].

Remark 3.3. S. Janson, [Ja2], has shown that boundedness of "big Hankel operators" is invariant under certain perturbations of the measure $\mu$, while the "small Hankel operators", which are the ones we will study in the next section, do not necessarily maintain their boundedness under these perturbations. At any rate the perturbations specified by Conditions (a) and (b) preceding Theorem 3.2 satisfy Janson's change of weight criteria while those specified by Conditions (c) and (d) in Theorem 3.2 do not. In both cases, however, our Theorem 4.5 will show that these perturbed measures and metrics lead to bounded "small Hankel operators."

Example 3.4 (The Riemann Surface for $z^{1 / n}$ ). Fix an integer $n \geq 2$. Denote by $M_{n}$ the $n$ sheeted Riemann surface associated to $z^{1 / n}$. Let $\mathbb{C}_{*}=$ $\mathbb{C}-\{0\}$. Then $M_{n}$ is a covering space of $\mathbb{C}_{*}$ with $n$ leaves. Let $\alpha: M_{n} \rightarrow \mathbb{C}_{*}$ be the covering map and let $g$ be the standard Riemannian metric on $M_{n}$. This is the metric that makes $\alpha_{*}$ an isometry at each point. That is, $g=d x^{2}+d y^{2}$ in the obvious local coordinates $x$ and $y$ lifted from $\mathbb{C}_{*}$. We take $\mu$ to be the measure on $M_{n}$ whose density with respect to the Riemann area element $d x d y$ is $(1 / n) p_{a}(\alpha(z))$ where $p_{a}(w)=(2 \pi a)^{-1} e^{-|w|^{2} / 2 a}$ for $w \in \mathbb{C}_{*}$. In other words we divide the Gaussian density $p_{a}$ equally among the $n$ sheets. Then $\mu$ is a probability measure on $M_{n}$. This example was extensively discussed in $[\mathbf{G} 4]$. It was shown in $[\mathbf{G} 4$, Section 6] that the triple $\left(M_{n}, g, \mu\right)$ is holomorphic and that our Standard Conditions hold. This example differs from the preceding ones, not only because of the different topology of the underlying manifold, but also because $\mathcal{H}^{2}$ is of codimension $n-1$ in $\mathcal{H} L^{2}(\mu)$. The functions $z^{-k / n} k=1,2, \ldots, n-1$, are in $\mathcal{H} L^{2}(\mu)$ but not in $\mathcal{H}^{2}$. The algebra $\mathcal{R}$ is spanned by the functions $z^{k / n}, k=0,1,2, \ldots$ on $M_{n}$. $\mathcal{R}$ is dense in all $\mathcal{H}^{p}$ but not in $\mathcal{H} L^{2}(\mu)$. The logarithmic Sobolev inequality (2.21) holds with $c=a$ and a constant $\beta \neq 0$. All the theorems of this paper are applicable to this example (with the exception of Corollary 4.18).

The next class of examples includes the exponential density const $\cdot e^{-|z| / a}$ on $\mathbb{C}$ and includes also some simple instances in which $\mathcal{H}^{2} \neq \mathcal{H} L^{2}$. The use 
of the exponential density in Section 5 will allow some explicit computations of Hilbert-Schmidt norms of Hankel operators (cf. Example 5.11).

Example 3.5. We take $M=\mathbb{C}-\{0\}$. We are going to use only radial densities. Let $\varphi$ be a strictly positive function in $C^{\infty}((0, \infty))$ such that $\varphi^{\prime}(s)<0$ for all $s>0$ and $\lim _{s \rightarrow \infty} \varphi(s)=0$. Define $w(z), \rho(z)$ and $\sigma(z)$ exactly as in (3.7)-(3.9) but only for $z \neq 0$. We will assume that $\rho$ is integrable over $M$ and that $b$ is chosen to normalize $\rho$. So $\int_{M} \rho(z) d x d y=1$. We will assume also that all of the momonts are finite: $\int_{M}|z|^{n} \rho(z) d x d y<$ $\infty$. Note that

$$
\varphi(s)=-\int_{s}^{\infty} \varphi^{\prime}(t) d t .
$$

So any strictly positive normalized radial density $\rho \in C^{\infty}(M)$ determines a function $\varphi$ by (3.8). (We take $b=1$ if $\rho$ is already normalized.) $\varphi$ in turn determines $w$ and $\sigma$. We define a metric on $M$ by (3.1). Then the triple $(\mathbb{C}-\{0\}, g, \rho d x d y)$ is holomorphic. The computation is the same as in Example 3.1. In particular, as in (3.12) and (3.13), we have

$$
Z=(2 / b) z \partial / \partial z, X=(2 / b) r \partial / \partial r, Y=(2 / b) \partial / \partial \theta
$$

in polar coordinates $(r, \theta)$. Clearly $Y$ is complete and Killing and $X$ is one-sided complete (even two sided complete). Thus Standard Conditions hold.

As to whether $\mathcal{H}^{2}=\mathcal{H} L^{2}$ we must distinguish between two essentially different cases determined by the behavior of $\rho$ near zero.

Proposition 3.6. Suppose that $\rho(z)$ is bounded near zero and that

$$
\int_{0<|z| \leq 1} \rho(z)|z|^{-2} d x d y=\infty .
$$

Then, writing $\mathcal{P}$ for the set of holomorphic polynomials on $\mathbb{C}$ restricted to $\mathbb{C}-\{0\}$, we have

$$
\mathcal{P} \subset \mathcal{H}^{2}=\mathcal{H} L^{2}
$$

Moreover $\mathcal{R}=\mathcal{P}$, which is dense in $\mathcal{H}^{2}$.

Proof. If $f \in \mathcal{H} L^{2}(M, \mu)$ then

$$
\infty>\int_{M}|f(z)|^{2} \rho(z) d x d y \geq \int_{0<|z| \leq 1}|f(z)|^{2} \rho(z) d x d y .
$$

Let $f(z)=\sum_{n=-\infty}^{\infty} a_{n} z^{n}$ be the Laurent expansion of $\mathrm{f}$ around $z=0$. For $0<\epsilon<1$ this series converges uniformly on the annulus $A_{\epsilon}=\{z$ : $\epsilon \leq|z| \leq 1\}$ and since $\rho$ is a radial function the terms $\left\{z^{n}\right\}_{n=-\infty}^{\infty}$ are mutually orthogonal in $L^{2}\left(A_{\epsilon}, \rho(z) d x d y\right)$. Therefore $\int_{A_{\epsilon}}|f(z)|^{2} \rho(z) d x d y=$ $\sum_{n=-\infty}^{\infty}\left|a_{n}\right|^{2} \int_{A_{\epsilon}}|z|^{2 n} \rho(z) d x d y$. As $\epsilon \downarrow 0$ the left side remains bounded while $\int_{A_{\epsilon}}|z|^{2 n} \rho(z) d x d y \rightarrow \infty$ for $n=-1,-2, \ldots$ by (3.18). Hence $a_{n}=0$ for 
$n \leq-1 . f$ therefore has a removable singularity at $z=0$. So any function in $\mathcal{H} L^{2}(M, \mu)$ is essentially an entire function.

Suppose now that $f \in \mathcal{P}$. By (3.16) $\varphi$ is bounded. Moreover it is shown in [G3, proof of Theorem 5.5] that $\phi(t) \leq c_{j} t^{-j}$ for some constants $c_{j}, j \geq 1$ and $t>0$. Consequently $\int_{M}|z|^{2 n} \phi\left(|z|^{2}\right) d x d y<\infty$ for $n \geq 0$. So $z^{n}$ has finite energy for all $n \geq 0$. Moreover $z^{n}$ is actually in $D(Q)$. A proof of this may be constructed by immitating the method of proof of $[\mathbf{G} 4$, Lemma 6.4]. (The hypothesis that $\rho$ is bounded near zero is used only at this stage of the proof and possibly could be elliminated by a more careful argument.) Now if $f$ is any entire function in $L^{2}(M, \mu)$ then the Fejer kernel argument of [G3, Lemma 5.4] shows that there is a sequence $p_{n} \in \mathcal{P}$ such that $p_{n} \rightarrow f$ in $L^{2}$. (One should choose $V_{\theta} f(z)=f\left(e^{i \theta} z\right)$ in that argument, just as in [G3, Lemma 5.4]. Thus $f \in \mathcal{H}^{2}$. So $\mathcal{H} L^{2} \subset \mathcal{H}^{2}$. This argument also shows that $\mathcal{P}$ is dense in $\mathcal{H}^{2}$. Finally, to show that $\mathcal{R}=\mathcal{P}$ observe that $Z z^{n}=(2 / b) z^{n} \in L^{2}$ for $n \geq 0$. Since $z^{n} \in \mathcal{H}^{2}$ we may apply [G3, Theorem 2.11 (e) and (b)] to conclude that $z^{n} \in D(A)$ and so is an eigenfunction of $A$. So $z^{n} \in \mathcal{R}$. Since $\mathcal{P}$ is dense in $\mathcal{H}^{2}$ these are all the eigenfunctions of $A$. Hence $\mathcal{R}=\mathcal{P}$.

Remark 3.7. If the equality (3.18) fails then $\mathcal{H}^{2} \neq \mathcal{H} L^{2}$. For example the function $f(z)=z^{-1}$ is clearly in $\mathcal{H} L^{2}$ if (3.18) fails. But this function cannot be in $\mathcal{H}^{2}$. To see this note that the equation preceding Equation (3.5) gives $Q(f)=\int_{M}\left|f^{\prime}(z)\right|^{2} w(z) d x d y$. So if $f(z)=z^{-1}$ then $Q(f)=$ $\int_{M}|z|^{-4} w(z) d x d y=\infty$ because $\varphi(s)$ is always bounded away from zero near $s=0$ by (3.16). So $f$ is not in $\mathcal{H} \cap D(Q)$. To show that $f$ cannot be approximated in $L^{2}$ norm by elements in $\mathcal{H} \cap D(Q)$ one can use the same argument used in the last paragraph of the proof of [G4, Lemma 6.4].

Thus, given that $\rho$ is bounded near zero, the equality (3.18) is necessary and sufficient for the equality $\mathcal{H}^{2}=\mathcal{H} L^{2}$.

Example 3.8. A special case of Example 3.5 which will be useful for computation is

$$
\rho(z)=c_{a} e^{-|z| / a}, z \neq 0
$$

where $c_{a}=\left(2 \pi a^{2}\right)^{-1}$ is the normalization constant. Choosing $b=1$ in (3.8) we have then $-\varphi^{\prime}(s)=c_{a} e^{-s^{1 / 2} / a}$. An integration from $s$ to $\infty$ gives $\varphi(s)=2 c_{a} a\left[s^{1 / 2}+a\right] e^{-s^{1 / 2} / a}$ for $s>0$. Hence from (3.9) we find

$$
\sigma(z)=2 a(|z|+a), z \neq 0 .
$$

In this example (3.18) holds. So $\mathcal{H}^{2}=\mathcal{H} L^{2}$ and $\mathcal{R}=\mathcal{P}$, which is an algebra. The reader might find it instructive to contemplate why $\mathcal{H}^{2}=\mathcal{H} L^{2}$ in this example but not in the Riemann surface example, Example 3.4, in spite of the fact that both manifolds are incomplete at zero and both have similar behavior of their densities near zero. 
We have not explored the validity of the logarithmic Sobolev inequality (2.21) in this example.

Example 3.9. (Lifts of measures on $0<|z|<\infty$ to the Riemann surface for $z^{1 / n}$.) Suppose that $\rho(z)$ and $\sigma(z)$ are given as in Example 3.5 and $d \nu(z)=\rho(z) d x d y$. Let $M_{n}$ be the Riemann surface for $z^{1 / n}$ as in Example 3.4 and let $\alpha: M_{n} \rightarrow \mathbb{C}_{*}$ be the natural covering map again. Define a probability measure $\mu$ on $M_{n}$ by $d \mu(z)=(1 / n) \rho(\alpha(z)) d x d y$ where $x$ and $y$ are again the local coordinates lifted from $\mathbb{C}_{*} . \mu$ is already normalized because of the factor $1 / n$ in the density since $M_{n}$ has $n$ leaves. Choose the Riemannian metric on $M_{n}$ which makes $\alpha_{*}$ an isometry from $T_{z}\left(M_{n}\right)$ to $T_{\alpha(z)}\left(\mathbb{C}_{*}\right)$ with the usual metric, $(3.1)$, namely $\sigma(\alpha(z))^{-1}\left(d x^{2}+d y^{2}\right)$, at $\alpha(z)$. Although we have changed the density from that in Example 3.5 by a factor $1 / n$ we are not changing $\sigma$. Equation (3.10), which is local, shows that the new triple $\left(M_{n}, g, \mu\right)$ is holomorphic and in fact that the new vector fields $X, Y, Z$ on $M_{n}$ are just the lifts of those on $\mathbb{C}_{*}$, already computed in Example 3.5, Equation (3.17). In the lifted version of (3.17) $z$ is to be interpreted as a point on $M_{n}$. So one should write, more properly, on $M_{n}$

$$
Z=(2 / b) \alpha(z) \partial / \partial z
$$

But we are going to write this in the following as $Z=(2 / b) z \partial / \partial z$ at the minor risk of confusing the point $z$ with the function $z(\equiv \alpha(z))$. There is no explicit $n$ dependence here because the factor $1 / n$ in $\rho$ cancels in (3.10) when $\sigma$ is fixed. One sees then easily that Standard Conditions hold and that $X$ is two sided complete on $M_{n}$.

The Example 3.4 is a special case of this class of examples. In that example one has $\rho(z)=$ const. $e^{-|z|^{2} / 2 a}$ and $\sigma(z) \equiv 1$. So down below, on $\mathbb{C}_{*}$ we have a Gaussian measure and the logarithmic Sobolev inequality (2.21) holds with $c=a$ and $\beta=0$. It is shown in [G4, Theorem 6.3] how the logarithmic Sobolev inequality $(2.21)$ can be lifted to $M_{n}$ with the same constant $c$ but with an increase in the size of $\beta$. The same lifting argument applies to the present class of examples. Of course one must already know that (2.21) holds down below, on $\mathbb{C}_{*}$.

An interesting aspect of this class of examples is that one may have $\mathcal{H}^{2}\left(\mathbb{C}_{*}, \nu\right)=\mathcal{H} L^{2}\left(\mathbb{C}_{*}, \nu\right)$ (see e.g., Proposition 3.6) and yet, for the induced metric and measure $\mu$ on $M_{n}$ this equation may fail over $M_{n}$. For example when $n=2$ the function $z^{-1 / 2}$ may be in $\mathcal{H} L^{2}\left(M_{2}, \mu\right)$, as it is in Example 3.4, but may not be in $\mathcal{H}^{2}\left(M_{2}, \mu\right)$, just as in Example 3.4. But the function $z^{-1 / 2}$ does not push down to a holomorphic function on $\mathbb{C}_{*}$ and therefore does not contribute to $\mathcal{H} L^{2}\left(\mathbb{C}_{*}, \nu\right)$.

As in the lifted Gaussian case, $\mathcal{R}$ is spanned by the functions $z^{k / n}, k=$ $0,1,2, \ldots$ and is therefore an algebra. This can be proved by the techniques 
of Proposition 3.6 in combination with the method of proof of [G4, Theorem $6.2]$.

Example 3.10 (Creation and annihilation operators over $M_{n}$ ). We are going to describe instances of Example 3.9 which are amenable to explicit computations of Hilbert-Schmidt norms of Hankel operators and for which the "creation" and "annihilation" operators play a useful role. These computations will be carried out in Example 5.11. As is well-known [Ba], [Se], in the space $\mathcal{H} L^{2}\left(\mathbb{C}, \gamma_{a}\right)$ the creation operator is given by multiplication by $z:(C f)(z)=z f(z)$ for $f \in \mathcal{H} L^{2}\left(\mathbb{C}, \gamma_{a}\right)$, with its natural domain, $\left\{f \in \mathcal{H} L^{2}\left(\mathbb{C}, \gamma_{a}\right): z f(z) \in L^{2}\right\}$. The adjoint of $C$ is computable. One finds $C^{*} f=2 a \partial f / \partial z$, with its natural domain. This simple form of $C^{*}$, just a differential operator, depends on the fact that one is using Gauss measure. For a general measure $\nu$ on $\mathbb{C}$, such as in Example 3.1 (with $m=1$ ) the adjoint of multiplication by $z$ may not be computable in a useful way. But the following lifted measures on $M_{n}$ give computable adjoints to the natural creation operators over $M_{n}$. Fix an integer $n \geq 2$ and let $a>0$. Define

$$
\rho(z)=c_{a} \exp \left(-|z|^{2 / n} / a\right), \quad z \in \mathbb{C}_{*}
$$

with $c_{a}$ chosen so that $\int_{\mathbb{C}} \rho(z) d x d y=1$. Let $d \nu(z)=\rho(z) d x d y$ and let $\mu$ be the lift of $\nu$ to $M_{n}$, as in Example 3.9. So $d \mu(z)=(1 / n) c_{a} e^{-|z|^{2 / n} / a} d x d y$ on $M_{n}$. Defining $\varphi$ by $(3.16)$ with $-\varphi^{\prime}(s)=c_{a} e^{-s^{1 / n} / a}$, the discussion in Example 3.9 shows that $\left(M_{n}, g, \mu\right)$ is a holomorphic triple when the metric $g$ is chosen as in that example. Moreover $Z$ is given on $M_{n}$ by (3.20) with $b=1$. So $Z=2 z \partial / \partial z$ on $M_{n}$. We reiterate that Standard Conditions hold in these examples and that $\mathcal{R}$ is an algebra. The natural analog of the creation operator in $\mathcal{H}^{2}\left(M_{n}, \mu\right)$ is multiplication by $z^{1 / n}$. Define

$$
C f(z)=z^{1 / n} f(z) \text { for } f \in \mathcal{H}^{2}\left(M_{n}, \mu\right) \text { and } z^{1 / n} f(z) \in \mathcal{H}^{2}\left(M_{n}, \mu\right) .
$$

This operator is easily shown to be closed on this domain and consequently has a densely defined adjoint, $C^{*}$, in $\mathcal{H}^{2}\left(M_{n}, \mu\right)$. We assert that

$$
\begin{gathered}
C^{*} C=(1 / 2) n a(A+2) \\
A C=C(A+(2 / n))
\end{gathered}
$$

and

$$
C^{*} C-C C^{*}=a\left(I+(n-1) P_{1}\right) \text { on } \mathcal{R}
$$

where $P_{1}$ is the projection of $\mathcal{H}^{2}\left(M_{n}, \mu\right)$ onto the one dimensional subspace consisting of the constant functions. The identities (3.23) and (3.24) will be useful in Example 5.11. The identity (3.25) is interesting because it captures the deviation from Gaussian, which is just the case $n=1$. Indeed, putting $n=1$ in (3.25) gives the canonical commutaton relations. 
Proof. Although the proof of these three identities can be given by direct computations over $M_{n}$, it is more perspicuous to transform the measure $\mu$ to an "almost" Gaussian measure. Let $\mathbb{C}^{*}=\mathbb{C}-\{0\}$ and define $\psi$ : $\mathbb{C}^{*} \rightarrow M_{n}$ by $\psi: \zeta \mapsto z=\zeta^{n}$. Then $\psi$ is a diffeomorphism. We have previously (in Example 3.4) denoted the set $\mathbb{C}-\{0\}$ by $\mathbb{C}_{*}$. But we wish to distinguish between the two distinct roles played by this set. Thus we have two maps $\mathbb{C}^{*} \rightarrow M_{n} \rightarrow \mathbb{C}_{*}$, the first being the diffeomorphism $\psi$, the second being the covering map $\alpha$. With $n$ and $a$ and $\nu$ and $\mu$ defined as in (3.21) and so on, define a probability measure $\hat{\mu}$ on $\mathbb{C}^{*}$ by $\hat{\mu}=\left(\psi^{-1}\right)_{*} \mu$. If $f$ is a nonnegative function on $\mathbb{C}^{*}$ then, writing $\zeta=\xi+i \eta$, the equations $\int_{\mathbb{C}^{*}} f(\zeta) d \hat{\mu}(\zeta)=\int_{M_{n}} f\left(\psi^{-1}(z)\right) d \mu(z)=\int_{M_{n}} f\left(z^{1 / n}\right) n^{-1} c_{a} e^{-|z|^{2 / n} / a} d x d y=$ $\int_{\mathbb{C}^{*}} f(\zeta) n^{-1} c_{a} e^{-|\zeta|^{2} / a} n^{2}|\zeta|^{2(n-1)} d \xi d \eta$ show that

$$
d \hat{\mu}(\zeta)=n c_{a} e^{-|\zeta|^{2} / a}|\zeta|^{2(n-1)} d \xi d \eta .
$$

See e.g., $\left[\mathbf{G} 4\right.$, Section 6] for the transformation $d x d y \mapsto n^{2}|\zeta|^{2(n-1)} d \xi d \eta$. The map $g \mapsto g \circ \psi$ is clearly unitary from $L^{2}\left(M_{n}, \mu\right)$ to $L^{2}\left(\mathbb{C}^{*}, \hat{\mu}\right)$. Moreover if $g \in L^{2}\left(M_{n}, \mu\right)$ then $g \in \mathcal{H}^{2}\left(M_{n}, \mu\right)$ if and only if $g \circ \psi$ has a removable singularity at $\zeta=0$ and so may be identified with an entire function on $\mathbb{C}$. This is proved in [G4, Lemma 6.4] for a similar measure. At a heuristic level this is already suggested by the fact that $\mathcal{H}^{2}\left(M_{n}, \mu\right)$ is spanned by the functions $z^{k / n}, k=0,1,2, \ldots$, which under composition with $\psi$ go to the functions $\zeta^{k}, k=0,1,2, \ldots$ We denote by $\mathcal{H}^{2}\left(\mathbb{C}^{*}, \hat{\mu}\right)$ the space of entire functions on $\mathbb{C}$ which are in $L^{2}\left(\mathbb{C}^{*}, \hat{\mu}\right)$ and we will prove the identities (3.23)(3.25) in this space. Incidentally, the notation $\mathcal{H}^{2}\left(\mathbb{C}^{*}, \hat{\mu}\right)$ for the indicated space is consistent with our previous notation for such spaces when the metric induced on $\mathbb{C}^{*}$ by $\psi$ is taken into account. But we will not need this. Define now $\hat{C}$ on $\mathcal{H}^{2}\left(\mathbb{C}^{*}, \hat{\mu}\right)$ by $\hat{C} f(\zeta)=\zeta f(\zeta)$ with its natural domain. Let $U f=f \circ \psi$ for $f \in \mathcal{H}^{2}\left(M_{n}, \mu\right)$. Then $U: \mathcal{H}^{2}\left(M_{n}, \mu\right) \rightarrow \mathcal{H}^{2}\left(\mathbb{C}^{*}, \hat{\mu}\right)$ is unitary and $U C=\hat{C} U$. The last equation just reflects the fact that $\mathrm{U}$ takes the function $z^{1 / n} f(z)$ to $\zeta f\left(\zeta^{n}\right)$. We will compute $\hat{C}^{*}$, using the "almost" Gaussian density (3.26). To this end note the identity

$$
\begin{aligned}
& \zeta e^{-|\zeta|^{2} / a}|\zeta|^{2(n-1)} \\
& =a\left[-(\partial / \partial \bar{\zeta})|\zeta|^{2(n-1)} e^{-|\zeta|^{2} / a}+(n-1)(1 / \bar{\zeta})|\zeta|^{2(n-1)} e^{-|\zeta|^{2} / a}\right] .
\end{aligned}
$$

Thus, for polynomials $f(\zeta)$ and $g(\zeta)$ we have

$$
\begin{aligned}
& \langle\hat{C} f, g\rangle_{L^{2}\left(\mathbb{C}^{*}, \hat{\mu}\right)} \\
& =n c_{a} \int_{\mathbb{C}^{*}} \zeta f(\zeta) \overline{g(\zeta)} e^{-|\zeta|^{2} / a}|\zeta|^{2(n-1)} d \xi d \eta \\
& =a n c_{a} \int_{\mathbb{C}^{*}} f(\zeta) \overline{\left(g^{\prime}(\zeta)+(n-1) \zeta^{-1} g(\zeta)\right)} e^{-|\zeta|^{2} / a}|\zeta|^{2(n-1)} d \xi d \eta
\end{aligned}
$$


by (3.27) and an easily justified integration by parts. If the polynomial $g$ has a nonzero constant term then $\zeta^{-1} g$ is no longer in $\mathcal{H}^{2}\left(\mathbb{C}^{*}, \hat{\mu}\right)$, although it is still in $\mathcal{H} L^{2}\left(\mathbb{C}^{*}, \hat{\mu}\right)$. One must therefore project it back into $\mathcal{H}^{2}$. Since $\zeta^{-1}$ is orthogonal to $\mathcal{H}^{2}$ one can accomplish this by just subtracting $\zeta^{-1} g(0)$. Equation (3.28) shows then that

$$
\left(\hat{C}^{*} g\right)(\zeta)=a\left[g^{\prime}(\zeta)+(n-1) \zeta^{-1}(g(\zeta)-g(0))\right] .
$$

Although (3.28) was carried out for polynomials $f$ these are easily seen to form a core for $\hat{C}$ and so (3.29) is indeed correct for all polynomials $g$. (The Gaussian case, $n=1$, gives the usual annihilation operator, $a \partial / \partial \zeta$.) Now (3.23) and (3.25) may be derived from (3.29). For a polynomial $g$ we have

$$
\left(\hat{C}^{*} \hat{C} g\right)(\zeta)=a[(\partial / \partial \zeta)(\zeta g(\zeta))+(n-1)(g(\zeta)-0)]=a\left[\zeta g^{\prime}(\zeta)+n g(\zeta)\right]
$$

while

$$
\left(\hat{C} \hat{C}^{*} g\right)(\zeta)=a\left[\zeta g^{\prime}(\zeta)+(n-1)(g(\zeta)-g(0))\right] .
$$

So $\left[\hat{C}^{*} \hat{C}-\hat{C} \hat{C}^{*}\right] g(\zeta)=a[g(\zeta)+(n-1) g(0)]$, which is $(3.25)$ because $\left(P_{1} g\right)(\zeta)$ $=g(0)$. To derive (3.23) we must first unitarily transform $A$ to an operator $\hat{A}$ on $\mathcal{H}^{2}\left(\mathbb{C}^{*}, \hat{\mu}\right)$ By the chain rule and $(3.20)$ (with $b=1$ ) one sees that

$$
(Z f) \circ \psi=\hat{Z}(f \circ \psi)
$$

where $\hat{Z}=(2 / n) \zeta \partial / \partial \zeta$. Thus (3.30) gives $\hat{C}^{*} \hat{C} g=(n a / 2)[\hat{Z} g+2 g]$ for polynomials $g$. But the self-adjoint operator $\hat{A}+2$ is diagonalized by the powers $\zeta^{k}, k \geq 0$, which form a core for $\hat{A}+2$. The self-adjoint operator $\hat{C}^{*} \hat{C}$ therefore extends the self-adjoint operator $\hat{A}+2$. Hence they are equal. This proves (3.23). Finally, the relation (3.24) follows from $(\hat{Z} \hat{C} f)(\zeta)=$ $(2 / n) \zeta(\partial / \partial \zeta)(\zeta f(\zeta))=\zeta[(\hat{Z} f)(\zeta)+(2 / n) f(\zeta)]=\hat{C}[\hat{Z}+(2 / n)] f(\zeta)$.

Example 3.11. Let $M=\{z \in \mathbb{C}:|z|<1\}$. It is easy to produce a general class of pairs $(g, \mu)$ on $M$ for which Standard Conditions hold: Choose a continuous function $\varphi:[0,1] \rightarrow[0, \infty)$ such that:

(1) $\varphi \in C^{\infty}([0,1))$,

(2) $\varphi>0$ on $[0,1)$, and

(3) $\varphi^{\prime}<0$ on $[0,1)$.

Define $g$ and $\mu$ by (3.1), (3.3), (3.7), (3.8) and (3.9), replacing of course $\mathbb{C}^{m}$ by $M$. Since $\int_{M} \rho(z) d x d y=2 \pi b[\varphi(1)-\varphi(0)]<\infty, \rho$ can be normalized by a suitable constant $b$. The same computations that lead to (3.11) now give $\nabla^{*} \nabla f(z)=(2 / b) z f^{\prime}(z)$ for $f \in \mathcal{H}(M)$. So $X=(2 / b) r \partial / \partial r$ and $Y=$ $(2 / b) \partial / \partial \theta$ in polar cordinates. Since $\sigma(z)$ is radial the $Y$ flow preserves the metric. Since $e^{-t X} z=e^{-2 t / b} z$ the $X$ flow is one sided complete. Thus Standard Conditions hold.

The second element of structure to be understood in this example is the purely functional anlytic question concerning the nature of the span, $\mathcal{R}$, of the eigenfunctions of $\nabla^{*} \nabla$ in $\mathcal{H}^{2}$. Proceedeing at an informal level for a 
moment, note that $\nabla^{*} \nabla z^{n}=(2 / b) z(d / d z) z^{n}=(2 / b) n z^{n}$ for all integers $n$. Since $\varphi^{\prime}(0)<0$ by assumption, we have $\rho(0)>0$. So $z^{n} \notin L^{2}(M, \rho d x d y)$ for $n \leq-1$. So the eigenfunctions of $\nabla^{*} \nabla$ in $\mathcal{H} L^{2}$ are, seemingly, the powers $z^{n}, n=0,1, \ldots$ One should therefore expect that $\mathcal{R}=\mathcal{P}$, the space of holomorphic polynomials on $M$. This is indeed correct, but only under additional conditions on $g$ and $\mu$ that are forced by considerations associated to the choice of boundary conditions that are needed to obtain a self-adjoint operator $A$. To understand the nature of this problem note first that $\nabla^{*} \nabla f(x)=-\sigma(x) \Delta f(x)+X f(x)$ for $f \in C^{\infty}(M)$ by a straightforward computation. Consider the special case $\sigma(x)=1, \rho=$ constant and therefore $X=0$. Standard Conditions hold in this case. But now $\nabla^{*} \nabla=-\Delta$, and the self-adjoint version of this operator corresponding to Dirichlet boundary conditions contains no nonzero holomorphic functions in its domain (or even in its form domain). It results that $\mathcal{H}^{2}=\{0\}$. Of course then $\mathcal{R}=\{0\}$ also. Similarly, if one chooses Neumann boundary conditions then $\mathcal{H}^{2}$ consists only of constant functions. Therefore neither of these two boundary conditions lead to an interesting theory when $\nabla^{*} \nabla=-\Delta$. (See [G4, Example 2.4] for further elaboration of this special case.) In order to obtain an interesting space $\mathcal{H}^{2}$ it is necessary that $\sigma(z) \rightarrow 0$ as $|z| \rightarrow 1$. In this case the elliptic operator $-\sigma(x) \Delta+X$ degenerates on the boundary $|z|=1$ and the first order term $X$ dominates the effect that boundary conditions have on the self-adjointness of $A$. The Keldysh-Fichera theory of such degeneracy has been intensively studied $[\mathbf{O R}]$. In the important case of the weighted Bergman spaces, where one has $\sigma(z)=$ const. $\left(1-|z|^{2}\right)$ and $\rho(z)=$ const. $\left(1-|z|^{2}\right)^{\gamma}$ with $\gamma>-1$, the effect of boundary conditions on $\mathcal{H}^{2}$ and on $\mathcal{R}$ has been analyzed, [G4, Section 5]. The next example surveys this instance in more detail. It will be used as the basis of a computational example in Section 5.

Example 3.12 (The weighted Bergman spaces $\mathcal{B}_{\gamma}$ ). Let $M=\{z \in \mathbb{C}$ : $|z|<1\}$. Define a metric on $M$ by

$$
g=\left(1-|z|^{2}\right)^{-1}(d x \otimes d x+d y \otimes d y)
$$

$g$ is not the usual hyperbolic metric. In fact $M$ is not complete in this metric. The dual metric is given by

$$
h(d z, d \bar{z})=2\left(1-|z|^{2}\right) .
$$

Let $-1<\gamma<\infty$ and define

$$
d \mu_{\gamma}(z)=a_{\gamma}\left(1-|z|^{2}\right)^{\gamma} d x d y, \quad \gamma>-1 .
$$

$\mu_{\gamma}$ is a finite measure on $M$ and $a_{\gamma}$ should be chosen to normalize $\mu_{\gamma}$. One can compute $\nabla^{*} \nabla f$ for a holomorphic function $f$ as in (2.14). One finds

$$
\left(\nabla^{*} \nabla f\right)(z)=2(\gamma+1) z f^{\prime}(z) \quad \text { for } \quad f \in \mathcal{H}(M), \gamma+1>0 .
$$


So $\nabla^{*} \nabla$ is holomorphic. The associated vector fields $Z, X$ and $Y$ are therefore given by

(3.34)

$$
Z=2(\gamma+1) z \partial / \partial z, X=2(\gamma+1)(x \partial / \partial x+y \partial / \partial y), Y=2(\gamma+1) \partial / \partial \theta .
$$

The associated flows are

$$
\exp (-t X) z=e^{-2(\gamma+1) t} z, \quad \exp (s Y) z=e^{2(\gamma+1) i s} z .
$$

The $Y$ flow is complete and consists of rotation about $z=0$. However the $X$ flow is only complete in one direction: For $t<0, e^{-2(\gamma+1) t} z$ reaches the boundary of $M$ in a finite (negative) time if $z \neq 0$.

For $0 \leq \gamma<\infty$ the Dirichlet boundary conditions defined in (2.6) and (2.7) yield a nonnegative self-adjoint operator $A$ to which our theory is applicable. One has $\mathcal{R}=\mathcal{P}$. So $\mathcal{R}$ is an algebra, which is of course dense in $\mathcal{H}^{2}$. But for $-1<\gamma<0$ one must use non-Dirichlet boundary conditions, whose need was explained in Example 3.11, to obtain the same result. However in all cases one has $A f(z)=2(\gamma+1) z f^{\prime}(z)$ and all holomorphic polynomials are in the domain of $A$. See [G4, Section 5] for details.

Hypercontractivity has only been proven for the weighted Bergman space with $\gamma=-1 / 2$ at the present time, [Ja1]. We believe that in fact supercontractivity holds in these spaces for all $\gamma>-1$. I.e., (2.21) holds for all $c>0$ for some $\beta$ depending on $c$. See e.g., $[\mathbf{G R}]$ for further discussion of this concept and its history. At any rate hypercontractivity by itself is not good enough to obtain anywhere near the best known results for these spaces. (See Remark 5.14.) For this reason we will pursue this example only for the purpose of explicit computations, in Example 5.13. Further development will require a better understanding of supercontractivity for these spaces.

Example 3.13. Let $M=\{z \in \mathbb{C}: 0<|z|<1\}$. This class of examples is similar to those in Example 3.11 except that we may now allow a singularity at the origin. Choose a continuous function $\varphi:[0,1] \rightarrow[0, \infty)$ satisfying:

(1) $\varphi \in C^{\infty}((0,1))$,

(2) $\varphi>0$ on $[0,1)$, and

(3) $\varphi^{\prime}<0$ on $(0,1)$.

Define $g, \mu, w, \rho, \sigma$ as in Example 3.11 again. Just as in Example 3.11 we see that Standard Conditions hold. But removal of the origin from $M$ now allows singular behavior near zero, such as e.g., $\varphi(s)=\left(1-s^{1 / 2}\right)^{\gamma+1},(\gamma>-1)$, which gives $\rho(z)=C_{1}(1-|z|)^{\gamma}|z|^{-1}$ and $\sigma(z)=C_{2}|z|(1-|z|)$ for some strictly positive constants $C_{1}$ and $C_{2}$. The allowed singularities at $z=0$ in $\rho$ and $\sigma$ can affect the behavior of functions in $\mathcal{H}^{2}$ and in $\mathcal{H} L^{2}$ in a way similar to that discussed in Example 3.5. The boundary at $|z|=1$, however, raises more serious technical problems concerning the useful boundary conditions for $A$. We expect that these problems will be similar to those already addressed in Example 3.12 as long as $\sigma(z) \rightarrow 0$ as $|z| \rightarrow 1$ and then one will have $\mathcal{R}=\mathcal{P}$. 
We have not explored this class of examples with respect to the question of whether the logarithmic Sobolev inequality (2.21) holds for some choices of $\varphi$.

Example 3.14. (Lifts of measures on $0<|z|<1$ to the Riemann surface for $z^{1 / n}$.) The measures and metrics described in Example 3.13 can be lifted up to that portion of the Riemann surface for $z^{1 / n}$ lying above $0<|z|<1$. The procedure is the same as that described in Example 3.9. One puts $1 / n$th of the density on each sheet and produces a holomorphic triple on the Riemann surface over $0<|z|<1$. However, just as in Examples 3.11 and 3.12, one must address the functional analytic question of boundary conditions at the outer boundary on the surface. We expect that the detailed theory worked out for the Bergman spaces, cf. Example 3.12, will be a reliable guide for what to expect for other measures.

Remark 3.15. There are transformations between different classes of examples which might sometimes provide technical help. For example the map $z \mapsto z^{2}$ from $\{0<|z|<\infty\}$ to the Riemann surface for $z^{1 / 2}$ is a diffeomorphism which maps the class of measures and metrics of Example 3.5 to the class of measures and metrics on the Riemann surface which are defined by a similar choice of function $\varphi$. Indeed a small part of the analysis of Example 3.4 was carried out in this way in [G4].

Example 3.16 (Product manifolds). Suppose that $\left(M_{1}, g_{1}, \mu_{1}\right)$ and $\left(M_{2}\right.$, $\left.g_{2}, \mu_{2}\right)$ both satisfy Standard Conditions. Let $M=M_{1} \times M_{2}, g=g_{1}+g_{2}$ and $\mu=\mu_{1} \times \mu_{2}$. It is sraightforward to verify that $(M, g, \mu)$ also satisfies Standard Conditions. And in fact $Z=Z_{1}+Z_{2}$. Moreover if the logarithmic Sobolev inequality (2.21) holds for each of the factors then it also holds for $(M, g, \mu)$ by the Faris additivity theorem, [G2, Theorem 2.3]. To be precise, if the constants in $(2.21)$ are $\left(c_{1}, \beta_{1}\right)$ and $\left(c_{2}, \beta_{2}\right)$, respectively, then the constants for the product manifold may be taken to be $\left(\max \left(c_{1}, c_{2}\right), \beta_{1}+\beta_{2}\right)$.

Example 3.17 (The Rudin ball). Let $M=\left\{z \in \mathbb{C}^{m}:|z|<1\right\}$. Define $g=\left(1-|z|^{2}\right)^{-1} \sum_{j=1}^{m}\left(d x_{j}^{2}+d y_{j}^{2}\right)$ where $z_{j}=x_{j}+i y_{j}$. Let $\gamma>-1$ and write $d \mu_{\gamma}(z)=b_{\gamma}\left(1-|z|^{2}\right)^{\gamma} \prod_{j=1}^{m} d x_{j} d y_{j}$ with normalization constant $b_{\gamma}$. Just as in the one dimensional case, (see Examples 3.11 and 3.12 and [G4, Section 5]) it is straightforward to show that Standard Conditions hold. (See also [G4, Remark 5.16].) In order to understand the structure of $\mathcal{H}^{2}$ and of $\mathcal{R}$ one must first study the boundary value problem for the associated degenerate elliptic operator, $-\left(1-|z|^{2}\right) \Delta+X$, in the ball. This has not been done in dimension $>1$. Nor has the validity of the logarithmic Sobolev inequality (2.21) been investigated in this case.

We close this section with an example that illustrates how it can happen that $\mathcal{R}$ is not closed under pointwise multiplication. 
Example 3.18 ( $\mathcal{R}$ may not be an algebra). Let $M=\mathbb{C}$ and let $\lambda>0$. Define

$$
\rho(z)=\frac{a \lambda}{\left(1+|z|^{2}\right)^{\lambda+1}} .
$$

Choose $a>0$ so that $d \mu(z)=\rho(z) d x d y$ is a probability measure. Let

$$
g=\lambda\left(1+|z|^{2}\right)^{-1}\left(d x^{2}+d y^{2}\right) .
$$

Now $Z$ is easily computable: $Z=2 z \partial / \partial z$. Consequently $X=2 r \partial / \partial r$ (in polar coordinates) and $Y=2 \partial / \partial \theta$. Therefore $e^{-t X} z=e^{-2 t} z$ and $e^{s Y} z=$ $e^{2 i s} z$. Hence $\nabla^{*} \nabla$ is holomorphic, $Y$ is complete and Killing, and $X$ is two sided complete. In particular, Standard Conditions hold. But this example is pathological for the study of Hankel operators. For in fact $\mathcal{H} L^{2}(\mu)$ is finite dimensional and is spanned by $\left\{1, z, z^{2}, \ldots, z^{m}\right\}$, where $m$ is the largest integer such that $\int_{\mathbb{C}}|z|^{2 m}\left(1+|z|^{2}\right)^{-\lambda-1} d x d y<\infty$. Hence $\mathcal{R}$ either consists only of the constant functions or else cannot be closed under multiplication. In the former case Equations (1.1) and (1.2) are uninteresting. In the latter case Equation (1.1) does not make sense. This example has been analyzed in more detail in [G3, Section 5]. It is shown there that $\mathcal{R}=\mathcal{H}^{2}=\mathcal{H} L^{2}(\mu)$. So if $m \geq 1$ then $\mathcal{R}$ is not an algebra.

One might seek to formulate the notion of Hankel form by insisting that (1.1) or (1.2) holds for $f$ and $g$ in $\mathcal{H} L^{4}(\mu)$. In this case $f g \in \mathcal{H} L^{2}(\mu)$, so both (1.1) and (1.2) make sense. But one must then address the question as to whether $\mathcal{H} L^{4}$ is dense in $\mathcal{H} L^{2}$ because, if not, then neither (1.1) nor (1.2) (with $\mathcal{R}$ replaced by $\mathcal{H} L^{4}$ ) determine the Hankel form $\Gamma$ uniquely on $\mathcal{H} L^{2} \times \mathcal{H} L^{2}$. In fact density of $\mathcal{H} L^{4}$ in $\mathcal{H} L^{2}$ does fail in the present example if $\lambda$ is chosen such that $\operatorname{dim} \mathcal{H} L^{4}=1$ while $\operatorname{dim} \mathcal{H} L^{2}>1$. Clearly such a choice of $\lambda$ is possible. Since $\mathcal{R}$ is always dense in $\mathcal{H}^{2}$ density issues disappear when we use the definitions (1.1) and (1.2) and insist that $\mathcal{R}$ be an algebra. We have no examples suggesting that this requirement on $\mathcal{R}$ represents any serious loss of generality. Moreover we note that examples like the present one are ruled out when Standard Conditions and the logarithmic Sobolev inequality (2.21) hold because then $\mathcal{H}^{p}$ is always dense in $\mathcal{H}^{q}$ for $0<q<p<\infty$ by [G3, Theorem 2.17], whereas in the present example dim $\mathcal{H}^{p}=1$ for large $p$ and $\operatorname{dim} \mathcal{H}^{2}>1$, at least when $\lambda>1$. Actually (2.21) fails in this example for all $\lambda$. See $[\mathbf{G} 3]$.

\section{Boundedness, compactness and Schatten classes.}

Throughout this section we will assume that the triple $(M, g, \mu)$ satisfies the Standard Conditions introduced in Notation 2.3 and that $\mathcal{R}$ is an algebra.

Our Hankel operators will act on the spaces $\mathcal{H}^{p}$ rather than on the sometimes larger spaces $\mathcal{H} L^{p}$ because our main tool, the hypercontractivity inequality (2.22), holds only for the spaces $\mathcal{H}^{p}$. 
Definition 4.1. Let $b \in L^{2}(\mu)$ and let $0<p, q<\infty$. By a Hankel form with symbol $b$ on $\mathcal{H}^{q} \times \mathcal{H}^{p}$ we mean a continuous bilinear form $\Gamma_{b}: \mathcal{H}^{q} \times \mathcal{H}^{p} \rightarrow \mathbb{C}$ which is given by

$$
\Gamma_{b}(f, g)=\langle f g, b\rangle \quad \forall f, g \in \mathcal{R} .
$$

When $p=q=2$ Equation (4.1) determines $\Gamma_{b}$ on all of $\mathcal{H}^{2} \times \mathcal{H}^{2}$ by continuity because $\mathcal{R}$ is always dense in $\mathcal{H}^{2}$. All our theorems dealing with $p$ or $q$ not equal to 2 will require that the logarithmic Sobolev inequality (2.21) hold. This will automatically assure that $\mathcal{R}$ is dense in both $\mathcal{H}^{p}$ and $\mathcal{H}^{q}$, [G3, Theorem 2.17]. Thus in all cases a function $b \in L^{2}$ will give rise to a Hankel form on $\mathcal{H}^{q} \times \mathcal{H}^{p}$ if and only if

$$
\left|\int_{M} \bar{b} f g d \mu\right| \leq \text { const. }\|f\|_{q}\|g\|_{p}, \quad \forall f, g \in \mathcal{R} .
$$

Our first goal will be to prove (4.2) under various regularity conditions on the symbol $b$. The present notion of a Hankel form clearly coincides with that in Section 1 when $p=q=2$ and $\mathcal{H}^{2}=\mathcal{H} L^{2}(\mu)$.

The main theorem of this paper is the following:

Theorem 4.2 (Boundedness of $\Gamma_{b}$ ). Assume that the logarithmic Sobolev inequality (2.21) holds. Let $b \in L^{2}(\mu)$. Suppose that $p \in(0, \infty), q \in(0, \infty)$, $r \in(1, \infty]$ and that $t \geq 0$ is such that

$$
e^{2 t / c} \geq r^{\prime}(1 / p+1 / q)
$$

where $r^{\prime}$ denotes the conjugate index to $r$. If

$$
b=e^{-t A} \phi \quad \text { for some } \phi \in L^{r}(\mu)
$$

then $\Gamma_{b}: \mathcal{H}^{q} \times \mathcal{H}^{p} \rightarrow \mathbb{C}$ is bounded and

$$
\left|\Gamma_{b}(f, g)\right| \leq M\left(p, p e^{2 t / c}\right) M\left(q, q e^{2 t / c}\right)\left\|e^{t A} b\right\|_{r}\|f\|_{q}\|g\|_{p} .
$$

( The constant $c$ is the one in (2.21) and $M(\cdot, \cdot)$ is defined in (2.24).)

Proof. Let $f, g \in \mathcal{R}$. Then $f g \in \mathcal{R} \subset L^{s}(\mu)$ for all $s \geq 1$. Since $e^{-t A}$ is Hermitian we have

$$
\Gamma_{b}(f, g)=\langle f g, b\rangle=\left\langle f g, e^{-t A} \phi\right\rangle=\left\langle e^{-t A}(f g), \phi\right\rangle=\left\langle\left(e^{-t A} f\right)\left(e^{-t A} g\right), \phi\right\rangle,
$$

wherein we have used (2.20). This implies, for $q_{0}, p_{0} \in(1, \infty)$ and $q_{0}^{-1}+$ $p_{0}^{-1}+r^{-1}=1$, that

$$
\left|\Gamma_{b}(f, g)\right| \leq\left\|e^{-t A} f\right\|_{q_{0}}\left\|e^{-t A} g\right\|_{p_{0}}\|\phi\|_{r} .
$$

Suppose first that equality holds in (4.3). Choose $q_{0}=q e^{2 t / c}$ and $p_{0}=p e^{2 t / c}$. Then $q_{0}^{-1}+p_{0}^{-1}+r^{-1}=(1 / q+1 / p) e^{-2 t / c}+r^{-1}$ which by (4.3) is the same 
as $1 / r^{\prime}+1 / r$ which equals 1 . In particular $q_{0}>1$ and $p_{0}>1$. Moreover, by strong hypercontractivity, (2.22), we have

$$
\left\|e^{-t A} f\right\|_{q_{0}} \leq M\left(q, q_{0}\right)\|f\|_{q}, \quad\left\|e^{-t A} g\right\|_{p_{0}} \leq M\left(p, p_{0}\right)\|g\|_{p} .
$$

Combining (4.7) with the last two inequalities gives

$$
\left|\Gamma_{b}(f, g)\right| \leq M\left(q, q_{0}\right) M\left(p, p_{0}\right)\|\phi\|_{r}\|f\|_{q}\|g\|_{p}
$$

which is (4.5) for $f$ and $g$ in $\mathcal{R}$. Since $\mathcal{R}$ is dense in $\mathcal{H}^{q}$ and $\mathcal{H}^{p}$ this estimate implies continuity of $\Gamma_{b}$ and the inequality (4.5) for the continuous extension of $\Gamma_{b}$. Next suppose that the right side of (4.3) is at least one, but inequality holds in (4.3). If $t_{0}$ is defined by $e^{2 t_{0} / c}=r^{\prime}(1 / p+1 / q)$ then $0 \leq t_{0}<t$. So (4.5) holds with $t$ replaced by $t_{0}$. But the first two factors in (4.5) are increasing functions of $t$, by the definition of $M(a, b)$. The third factor is also an increasing function of $t$ because $\left\|e^{t_{0} A} b\right\|_{r}=\left\|e^{\left(t_{0}-t\right) A} e^{t A} b\right\|_{r} \leq\left\|e^{t A} b\right\|_{r}$. Hence (4.5) holds if $t$ satisfies (4.3) and $r^{\prime}(1 / p+1 / q) \geq 1$.

Finally, suppose that $r^{\prime}(1 / p+1 / q)<1$. Then $1 / p+1 / q<1 / r^{\prime}=1-1 / r$. So $1 / p+1 / q+1 / r<1$. Clearly $p$ and $q$ must both be greater than one. We may choose $q_{0} \in(1, q)$ and $p_{0} \in(1, p)$ such that $p_{0}^{-1}+q_{0}^{-1}+r^{-1}=1$. Now $\|f\|_{q_{0}} \leq\|f\|_{q}$ and $\|g\|_{p_{0}} \leq\|g\|_{p}$ because $\mu$ is a probability measure. So Hölder's inequality gives $\left|\Gamma_{b}(f, g)\right| \leq\|f\|_{q_{0}}\|g\|_{p_{0}}\|b\|_{r} \leq\|f\|_{q}\|g\|_{p}\|b\|_{r}$ which is (4.5) with $t=0$. The previous monotonicity argument in $t$ now applies.

Remark 4.3. The conclusion of Theorem 4.2 is strongest for small $t$ because smaller $t$ imposes less regularity on $b$. Thus equality in (4.3) will be of most interest, provided $t \geq 0$. Moreover, by (4.3), small $r$ forces large $t$. The hypothesis that $\left\|e^{t A} b\right\|_{r}$ is finite allows one to balance regularity of $b$ (large $t$ ) against large size (small $r$ ).

Corollary $4.4\left(H_{b}: \mathcal{H}^{p} \rightarrow \mathcal{H}^{2}\right.$ is bounded). Assume that (2.21) holds. Let $b \in L^{2}(\mu)$ and let $1<r \leq \infty$. Suppose that $0<p<\infty$ and that $e^{2 t / c}=$ $r^{\prime}(1 / p+1 / 2)$ for some $t \geq 0$. If $b=e^{-t A} \phi$ for some $\phi \in L^{r}(\mu)$ then $H_{b}:$ $\mathcal{H}^{p} \rightarrow \mathcal{H}^{2}$ is bounded. Moreover

$$
\left\|H_{b}\right\|_{\mathcal{H}^{p} \rightarrow \mathcal{H}^{2}} \leq M\left(p, p e^{2 t / c}\right) M\left(2,2 e^{2 t / c}\right)\left\|e^{t A} b\right\|_{r} .
$$

Theorem $4.5\left(H_{b}: \mathcal{H}^{2} \rightarrow \mathcal{H}^{2}\right.$ is bounded). Assume that $(2.21)$ holds. Let $b \in L^{2}(\mu)$ and suppose that $1<r \leq \infty$. If, for some $\phi \in L^{r}(\mu), b=e^{-t A} \phi$ with $e^{2 t / c}=r^{\prime}$ then $H_{b}: \mathcal{H}^{2} \rightarrow \mathcal{H}^{2}$ is bounded and

$$
\left\|H_{b}\right\|_{\mathcal{H}^{2} \rightarrow \mathcal{H}^{2}} \leq M\left(2,2 r^{\prime}\right)^{2}\left\|e^{t A} b\right\|_{r} .
$$

Proofs of Corollary 4.4 and Theorem 4.5. When $q=2$ the inequality (4.5) is equivalent to (4.10) because $\mathcal{H}^{2}$ is self dual. When $p$ is also equal to two the condition on $t$ in Corollary 4.4 reads $e^{2 t / c}=r^{\prime}$. So (4.10) reduces to (4.11). 
We note for later use that the relation $e^{2 t / c}=r^{\prime}$ may be restated as $t=$ $t_{J}\left(2,2 r^{\prime}\right)=\frac{c}{2} \log \frac{2 r^{\prime}}{2}$, by (2.23). Furthermore (4.11) holds for $t \geq t_{J}\left(2,2 r^{\prime}\right)$ in view of (4.3). But the shortest time imposes the least regularity on $b$ for a given exponent $r$.

Two simple subcases may be illuminating. First, take $r=\infty$ in Theorem 4.5. Then $r^{\prime}=1$ and so $t=0$. Hence $b=\varphi$, which is in $L^{\infty}(M, \mu)$. The coefficient in (4.11) is now equal to one. We therefore find $\left\|H_{b}\right\|_{\mathcal{H}^{2} \rightarrow \mathcal{H}^{2}} \leq$ $\|b\|_{\infty}$. That is, a bounded symbol gives a bounded Hankel operator on $\mathcal{H}^{2}$, which is well-known. Second, in Corollary 4.4, again take $r=\infty$. Then, in order to obtain a bounded Hankel operator from $\mathcal{H}^{p}$ into $\mathcal{H}^{2}$, Corollary 4.4 requires that one imposes some regularity on the bounded symbol $b$ if $p<2$ but not if $p>2$.

In Example 4.15 we will show how it can be useful to play regularity against size ( $t$ against $r$ ) and at the same time we will make comparison of Theorem 4.5 with the known best results, $[\mathbf{J P R}]$, in the Gaussian case. Moreover estimates which are converse to (4.11), and thus give necessary regularity conditions on $b$, are discussed in Remark 6.11.

Note that when $H_{b}: \mathcal{H}^{2} \rightarrow \mathcal{H}^{2}$ is bounded one has the symmetry

$$
\left\langle f, H_{b} g\right\rangle=\left\langle g, H_{b} f\right\rangle \quad \forall f, g \in \mathcal{H}^{2}
$$

because this identity holds for all $f, g \in \mathcal{R}$ by (4.1) and $\mathcal{R}$ is dense in $\mathcal{H}^{2}$.

Next we investigate the trace ideal properties and compactness of $H_{b}$ : $\mathcal{H}^{2} \rightarrow \mathcal{H}^{2}$. The identity (4.13) is the key for our results. We postpone examples to Section 5 where we investigate the Hilbert-Schmidtness of $H_{b}$ in greater detail. The invariance expressed in (4.13) has been explored already in the classical contexts by J. Peetre.

Theorem 4.6. If $b \in L^{2}(\mu)$ and $H_{b}$ is bounded on $\mathcal{H}^{2}$ then, for any $t \geq 0$, $H_{e^{-t A} b}$ is bounded on $\mathcal{H}^{2}$ and is given by

$$
H_{e^{-t A b}}=e^{-t A} H_{b} e^{-t A} \quad \text { on } \mathcal{H}^{2} .
$$

Proof. For $f, h \in \mathcal{R}$ consider the bilinear form

$$
\begin{aligned}
\Gamma(f, h) & :=\int_{M} \overline{e^{-t A} b} f h d \mu \\
& =\int_{M} \bar{b} e^{-t A}(f h) d \mu \\
& =\int_{M} \bar{b}\left(e^{-t A} f\right)\left(e^{-t A} h\right) d \mu \\
& =\left\langle e^{-t A} f, H_{b} e^{-t A} h\right\rangle \\
& =\left\langle f, e^{-t A} H_{b} e^{-t A} h\right\rangle .
\end{aligned}
$$


Since $\mathcal{R}$ is dense in $\mathcal{H}^{2}$, and $e^{-t A}: \mathcal{H}^{2} \rightarrow \mathcal{H}^{2}$ is bounded we see that $\Gamma$ extends to a continuous bilinear form on $\mathcal{H}^{2}$. The first line in (4.14) shows that $\Gamma$ has symbol $e^{-t A} b$, and the last line shows that (4.13) holds.

Let us recall that for $p>0$ a compact Hermitian operator $T$ with eigenvalues $\left\{\lambda_{k}\right\}$ (counting multiplicity) is in the Schatten class $S_{p}$ if $\sum_{k=0}^{\infty}\left|\lambda_{k}\right|^{p}<$ $\infty$.

Corollary 4.7 (Schatten class). Let $p>0$. If $e^{-2 t A}$ is in the Schatten class $S_{p}$ and $H_{b}$ is bounded on $\mathcal{H}^{2}$ then $H_{e^{-t A} b} \in S_{p}$ on $\mathcal{H}^{2}$.

Proof. If $p_{1}, p_{2}, p>0$ satisfy $p_{1}^{-1}+p_{2}^{-1}=p^{-1}$ and $T_{1} \in S_{p_{1}}, T_{2} \in S_{p_{2}}$ we have [DS, p. 1093] $\left\|T_{1} T_{2}\right\|_{p} \leq 2^{\frac{1}{p}}\left\|T_{1}\right\|_{p_{1}}\left\|T_{2}\right\|_{p_{2}}$. Together with the ideal property of $S_{p}$ this implies

$$
\begin{aligned}
\left\|e^{-t A} H_{b} e^{-t A}\right\|_{S_{p}} & \leq 2^{\frac{1}{p}}\left\|e^{-t A}\right\|_{S_{2 p}}\left\|H_{b} e^{-t A}\right\|_{S_{2 p}} \\
& \leq 2^{\frac{1}{p}}\left\|e^{-t A}\right\|_{S_{2 p}}^{2}\left\|H_{b}\right\|_{o p} \\
& =2^{\frac{1}{p}} \operatorname{tr}\left(e^{-2 p t A}\right)^{1 / p}\left\|H_{b}\right\|_{o p}<\infty .
\end{aligned}
$$

The corollary now follows from (4.13).

Theorem 4.8 (Compactness of $H_{b}$ ). Assume that (2.21) holds. Suppose that $1<r<\infty$ and that $e^{2 t / c}=r^{\prime}$. (Equivalently, $t=t_{J}\left(2,2 r^{\prime}\right)$.) If $b \in L^{2}(\mu)$ and $b=e^{-t A} \phi$ for some function $\phi \in L^{r}$ then $H_{b}: \mathcal{H}^{2} \rightarrow \mathcal{H}^{2}$ is compact.

Proof. By Theorem $4.5 H_{b}$ is a bounded operator from $\mathcal{H}^{2}$ into $\mathcal{H}^{2}$. Moreover Theorem 4.6 shows that $H_{e^{-\epsilon A}}=e^{-\epsilon A} H_{b} e^{-\epsilon A}$. By the Bogachev-RocknerZhang-Cipriani-Wang theorem $[\mathbf{B R Z}, \mathbf{C i}, \mathbf{W a}] A$ has compact resolvent in $L^{2}$. Therefore $e^{-\epsilon A}$ is a compact operator in $L^{2}$ for each $\epsilon>0$. It follows that $H_{e^{-\epsilon A b}}$ is compact for each $\epsilon>0$. Now $e^{-\epsilon A} b=e^{-t A} e^{-\epsilon A} \phi$. Since $e^{-\epsilon A} \phi$ is also in $L^{r}$ Theorem 4.5 gives

$$
\left\|H_{b}-H_{e^{-\epsilon A} b}\right\|_{o p}=\left\|H_{b-e^{-\epsilon A} b}\right\|_{o p} \leq\left\|\left(I-e^{-\epsilon A}\right) \phi\right\|_{r} M\left(2,2 r^{\prime}\right)^{2},
$$

which goes to zero as $\epsilon \rightarrow 0$ because the semigroup $e^{-s A}$ is strongly continuous in $L^{r}$.

Note that the case $r=\infty$ is excluded from the compactness theorem, Theorem 4.8, even though it is not excluded from the boundedness theorem, Theorem 4.5. The reason for this is that the contraction semigroup $e^{-s A}$ is usually not strongly continuous on $L^{\infty}(\mu)$. So the last step in the proof of Theorem 4.8 breaks down. But if $\phi$ is in the closed subspace of $L^{\infty}$ on which $e^{-s A}$ is strongly continuous then the previous proof applies with no change. Of course $t=0$ when $e^{2 t / c}=r^{\prime}=1$. We state this as a corollary. 
Corollary 4.9 (Compactness of $H_{b}$ ). Assume that (2.21) holds. Suppose that $b \in L^{\infty}(\mu)$ and that $\left\|e^{-\epsilon A} b-b\right\|_{\infty} \rightarrow 0$ as $\epsilon \downarrow 0$. Then $H_{b}$ is a compact operator on $\mathcal{H}^{2}$.

Lemma 4.10. Suppose that $H_{b}$ is bounded on $\mathcal{H}^{2}$ and let $0<r<\infty$. Then, for $f, h \in \mathcal{H}^{2}$ :

$$
\left|\left\langle f, H_{(A+2)^{-2 r} b} h\right\rangle\right| \leq 2^{-2 r}\left\|H_{b}\right\|_{o p}\left\|(A+1)^{-r} f\right\|_{2}\left\|(A+1)^{-r} h\right\|_{2} .
$$

Proof. The gamma function satisfies the identity

$$
\Gamma(2 r) t^{-2 r}=\int_{0}^{\infty} s^{2 r-1} e^{-s t} d s,
$$

which is valid for $t>1$. Thus, for $f \in L^{2}(\mu)$ we have

$$
\Gamma(2 r)(A+2)^{-2 r} f=\int_{0}^{\infty} s^{2 r-1} e^{-s(A+2)} f d s .
$$

Notice that $(A+2)^{-2 r} b \in L^{2}(\mu)$ because $r>0$. We therefore have, for $f$ and $h$ in $\mathcal{R}$ :

$$
\begin{aligned}
\Gamma(2 r)\left\langle f, H_{(A+2)^{-2 r} b} h\right\rangle & =\Gamma(2 r)\left\langle f h,(A+2)^{-2 r} b\right\rangle \\
& =\int_{0}^{\infty} s^{2 r-1}\left\langle f h, e^{-s(A+2)} b\right\rangle d s \\
& =\int_{0}^{\infty} s^{2 r-1}\left\langle\left(e^{-s(A+1)} f\right)\left(e^{-s(A+1)} h\right), b\right\rangle d s \\
& =\int_{0}^{\infty} s^{2 r-1}\left\langle e^{-s(A+1)} f, H_{b} e^{-s(A+1)} h\right\rangle d s .
\end{aligned}
$$

Hence

$$
\begin{aligned}
& \Gamma(2 r)\left|\left\langle f, H_{(A+2)^{-2 r} b} h\right\rangle\right| \\
& \leq \int_{0}^{\infty} s^{2 r-1}\left\|e^{-s(A+1)} f\right\|_{2}\left\|e^{-s(A+1)} h\right\|_{2}\left\|H_{b}\right\|_{o p} d s \\
& \leq\left\|H_{b}\right\|_{o p}\left\{\int_{0}^{\infty} s^{2 r-1}\left\|e^{-s(A+1)} f\right\|_{2}^{2} d s \int_{0}^{\infty} s^{2 r-1}\left\|e^{-s(A+1)} h\right\|_{2}^{2} d s\right\}^{\frac{1}{2}} .
\end{aligned}
$$

But

$$
\begin{aligned}
\int_{0}^{\infty} s^{2 r-1}\left\|e^{-s(A+1)} f\right\|_{2}^{2} d s & =\int_{0}^{\infty} s^{2 r-1}\left\langle e^{-2 s(A+1)} f, f\right\rangle d s \\
& =\Gamma(2 r)\left\langle(2(A+1))^{-2 r} f, f\right\rangle \\
& =\Gamma(2 r) 2^{-2 r}\left\|(A+1)^{-r} f\right\|_{2}^{2} .
\end{aligned}
$$

Using this identity in (4.18) twice we get (4.17) for $f, h \in \mathcal{R}$. Since $\mathcal{R}$ is dense in $\mathcal{H}^{2}$ it follows that $H_{(A+2)^{-2 r} b}$ has a continuous extension to $\mathcal{H}^{2}$ and that (4.17) holds for all $f, h \in \mathcal{H}^{2}$. 
Theorem 4.11 (Schatten class). Suppose that $b \in L^{2}(\mu)$ and that $H_{b}$ is bounded on $\mathcal{H}^{2}$. Let $r>0$ and $p>0$. If $(A+1)^{-2 r}$ is in $S_{p}$ then $H_{(A+2)^{-2 r} b}$ is in $S_{p}$.

Proof. By (4.17) there is a bounded operator $B$ on $\mathcal{H}^{2}$ such that

$$
H_{(A+2)^{-2 r} \varphi}=(A+1)^{-r} B(A+1)^{-r} .
$$

The assertion now follows as in the proof of Corollary 4.7.

Combining this theorem with Theorem 4.5 yields:

Corollary 4.12. Assume that (2.21) holds. Suppose that $1<r<\infty$, that $\phi \in L^{r}(\mu)$ and that for some $s>0(A+1)^{-s}$ is in $S_{p}$. Let

$$
b:=(A+2)^{-s} e^{-t_{J}\left(2,2 r^{\prime}\right) A} \phi .
$$

Then $H_{b}$ is in the Schatten class $S_{p}$ on $\mathcal{H}^{2}$.

Notation 4.13. (Naturally induced measures and regularity of holomorphic symbols.) It is sometimes possible to express regularity conditions on a holomorphic symbol $b$ by a size condition on $b$ with respect to a different measure. Suppose that the vector field $X$ associated to $(M, g, \mu)$ is 2-sided complete. The diffeomorphism group $t \mapsto \exp (t X)$ induces a family of probability measures $\mu_{t}$ on $M$ defined by

$$
\mu_{t}=(\exp t X)_{*} \mu \quad t \in \mathbb{R} .
$$

Of course even if $X$ is only one sided complete the measures $\mu_{t}$ given in (4.20) are well-defined probability measures on $M$ for $t \leq 0$. But our interest in these measures lies in their usefulness for describing regularity of symbols $b$, and for this we need $t>0$. If, for example, $\mu$ is Gauss measure on $\mathbb{C}^{m}$ then $\mu_{t}$ is again Gauss measure but with a different covariance. Indeed the measure $\left(\gamma_{a}\right)_{t} \equiv\left(e^{t X}\right)_{*} \gamma_{a}$ is easily computed. Taking the notation from Example 2.4, we have $e^{t X} z=e^{t / a} z$. If $f \in C_{c}\left(\mathbb{C}^{m}\right)$ then, making the change of variables $z=e^{t / a} w$, we have $\int f(w) d\left(\gamma_{a}\right)_{t}(w)=\int f\left(e^{t X} w\right) p_{a}(w) d w=$ $\int f(z) p_{a}\left(e^{-t / a} z\right) \operatorname{det}\left(e^{-t / a} I\right) d z=\int f(z) p_{a e^{2 t / a}}(z) d z$. Hence

$$
\left(e^{t X}\right)_{*} \gamma_{a}=\gamma_{a e^{2 t / a}} \quad t \in \mathbb{R}
$$

Although the vector field $X$ is 2-sided complete in all of the examples of Section 3 except Examples 3.11-3.13, the induced measures $\mu_{t}$ may not be perspicuously related to $\mu$. Another class of examples for which the transformed measure is usefully computable is that described in Example 3.10. Denote by $\nu_{a}$ the measure on $\mathbb{C}_{*}$ with density (3.21) and by $\mu_{a}$ its lift to $M_{n}$. These measures were denoted simply by $\nu$ and $\mu$ in Example 3.10 for ease in reading. The vector field $X$ in all these examples is given by (3.17), or its lift, as in (3.20) (with $b=1$ ) because $\rho$ was already normalized. Hence 
$X=2 r \partial / \partial r$ in these cases and $e^{t X} z=e^{2 t} z$. A computation similar to the previous Gaussian computation now gives

$$
\left(e^{t X}\right)_{*} \nu_{a}=\nu_{a e^{4 t / n}}
$$

and

$$
\left(e^{t X}\right)_{*} \mu_{a}=\mu_{a e^{4 t / n}}
$$

Proposition 4.14. Assume that $X$ is two sided complete. Let $t>0$ and suppose that $b \in D\left(e^{t A} \mid \mathcal{H}^{2}\right)$. Then

$$
\left\|e^{t A} b\right\|_{L^{p}(\mu)}=\|b\|_{L^{p}\left(\mu_{t}\right)} \quad 0<p<\infty,
$$

which can be infinite.

Proof. By assumption there is a function $f$ in $\mathcal{H}^{2}$ such that $b=e^{-t A} f$. So $b(z)=f\left(e^{-t X} z\right)$ for all $z$ in $M$ by (2.19). Therefore $f(z)=b\left(e^{t X} z\right)$. Hence

$$
\left\|e^{t A} b\right\|_{L^{p}(\mu)}^{p}=\|f\|_{L^{p}(\mu)}^{p}=\int_{M}\left|b\left(e^{t X} z\right)\right|^{p} d \mu(z)=\int_{M}|b(z)|^{p} d \mu_{t}(z) .
$$

The proof shows that (4.24) holds also for $t<0$ if $b \in \mathcal{H}^{2}$. One need not introduce the function $f$ into the proof in that case. For $t>0$ Equation (4.24) shows that the extremely strong regularity condition $\left\|e^{t A} b\right\|_{L^{p}(\mu)}<\infty$, which we have imposed on the Hankel symbol $b$ in Equation (4.4), can be formulated as a simple $L^{p}$ condition with respect to a different measure if $b \in \mathcal{H}^{2}$. We will see this phenomenon recurring in several examples in Section 5. The conversion of regularity of the holomorphic symbol $b$ to size of $b$ by Equation (4.24) provides some perspective on the conditions used in [JPR] to obtain bounds on Hankel operators in the Gaussian case.

Example 4.15 (Gauss measure on $\mathbb{C}^{m}$. Comparison with $[\mathbf{J P R}]$ ). Referring to Example 2.4, we note that all theorems of this section apply to this case with $M(\cdot, \cdot)=1$ in Equations (4.5), (4.10) and (4.11) because $\beta=0$. Since $X$ is two-sided complete, Equations (4.21) and (4.24) allow one to formulate the conclusions in Theorem 4.2 and its corollaries in terms of $L^{r}$ norms of the symbol $b$ when $b$ is holomorphic. Consider the simplest and most important case, Theorem 4.5. In the two subcases discussed after Theorem 4.5 we took $r=\infty$, which is inappropriate when $b$ is holomorphic because $b$ must be constant if it is both holomorphic and bounded. Suppose then that $1<r<\infty$ and that $b \in \mathcal{H} L^{2}\left(\gamma_{a}\right)$. If $e^{2 t / a}=r^{\prime}$ and $e^{t A} b \in L^{r}\left(\gamma_{a}\right)$ then Theorem 4.5 asserts that $H_{b}: \mathcal{H} L^{2}\left(\gamma_{a}\right) \rightarrow \mathcal{H} L^{2}\left(\gamma_{a}\right)$ is bounded and the inequality (4.11) holds. But $\left\|e^{t A} b\right\|_{L^{r}\left(\gamma_{a}\right)}=\|b\|_{L^{r}\left(\gamma_{a r^{\prime}}\right)}$ by (4.21) and (4.24). Thus we may conclude that

$$
\left\|H_{b}\right\|_{\mathcal{H} L^{2}\left(\gamma_{a}\right) \rightarrow \mathcal{H} L^{2}\left(\gamma_{a}\right)} \leq\|b\|_{L^{r}\left(\gamma_{a r^{\prime}}\right)}, \quad 1<r<\infty, \quad b \in \mathcal{H} L^{2}\left(\gamma_{a}\right) .
$$


The left side of (4.25) does not depend on $r$. On the right side we have incorporated the $t$ dependence into the variance $a r^{\prime} . r$ is still at our disposal. For a particular holomorphic symbol $b$ it is of course desirable to choose $r$ so as to minimize $\|b\|_{L^{r}\left(\gamma_{a r^{\prime}}\right)}$ or at least so as to make $\|b\|_{L^{r}\left(\gamma_{a r^{\prime}}\right)}$ finite. Take $m=1$ for simplicity of writing and define $b(z)=e^{s z^{2}}$ for some $s>0$. Then, for any $v>0$, we have $\|b\|_{L^{r}\left(\gamma_{v}\right)}^{r}=$ const. $\int_{\mathbb{C}} e^{r s\left(x^{2}-y^{2}\right)} e^{-\left(x^{2}+y^{2}\right) /(2 v)} d x d y$. So

$$
\|b\|_{L^{r}\left(\gamma_{v}\right)}<\infty \text { if and only if } r s<(2 v)^{-1} .
$$

In particular, putting $v=a r^{\prime}$, we see that $\|b\|_{L^{r}\left(\gamma_{a r^{\prime}}\right)}<\infty$ if and only if $s<\left(2 a r^{\prime} r\right)^{-1}$. Now $\left(r^{\prime} r\right)^{-1}=(r-1) / r^{2}$, which achieves a maximum at $r=2$. Thus the weakest restriction on $s$ is achieved at $r=2$. For $r=2$ the last inequality says $s<(8 a)^{-1}$. Therefore for the class of functions $b(z)=e^{s z^{2}}$ the best we can deduce from Theorem 4.5 is that $H_{b}$ is bounded on $\mathcal{H}^{2}\left(\gamma_{a}\right)$ if $s<(8 a)^{-1}$. We may compare this with the known best result in the Gaussian case, [JPR, Theorem 7.5, (a), with $\beta=\alpha=(2 a)^{-1}$ and $p=\infty$ ], which shows that $H_{b}$ is bounded on $\mathcal{H}^{2}\left(\gamma_{a}\right)$ if and only if $s \leq(8 a)^{-1}$. Thus our general method fails to achieve the known boundedness of $H_{b}$ for the limiting value $s=(8 a)^{-1}$.

The previous computations for $b(z)=e^{s z^{2}}$ also show that the lower bound on $t$, given by the inequality (4.3), is best, at least in the case $p=q=$ $r=2$. Indeed with these values (4.3) reads $e^{2 t / a} \geq 2$ (since $\mathrm{c}=\mathrm{a}$ in the present example). To see that this inequality is a necessary condition for boundedness of $H_{b}$ on $\mathcal{H}^{2}$ suppose that $\alpha \equiv e^{2 t_{0} / a}<2$ and that $t_{0} \geq 0$. Since $\left\|e^{t_{0} A} b\right\|_{L^{2}\left(\gamma_{a}\right)}=\|b\|_{L^{2}\left(\gamma_{a \alpha}\right)}$ by (4.21) and (4.24), the assertion (4.26) (with $v=a \alpha$ ) shows that $\left\|e^{t_{0} A} b\right\|_{L^{2}\left(\gamma_{a}\right)}<\infty$ if and only if $s<(4 a \alpha)^{-1}$. Since $\alpha<2$ there is an $s$ satisfying $(8 a)^{-1}<s<(4 a \alpha)^{-1}$. For such a value of $s H_{b}$ is unbounded on $\mathcal{H}^{2}\left(\gamma_{a}\right)$ (by [JPR, Theorem 7.5, (a)]) even though $\left\|e^{t_{0} A} b\right\|_{L^{2}\left(\gamma_{a}\right)}<\infty$. Thus the lower bound on $t$ specified by (4.3) cannot be improved in this Gaussian example. See also Remark 6.11 for another proof that (4.3) cannot be improved.

Remark 4.16 (Target space $=\mathcal{H}^{q^{\prime}}$ ). We have focused so far on the Hankel operator $H_{b}$ as an operator on $\mathcal{H}^{2}$ (in Theorem 4.5) and more generally as an operator from $\mathcal{H}^{p}$ into $\mathcal{H}^{2}$ (in Corollary 4.4). But the bound (4.5) also offers the possibility of establishing boundedness of $H_{b}$ as an operator from $\mathcal{H}^{p}$ into $\mathcal{H}^{q^{\prime}}$ when $q \in(1, \infty)$ and $q^{\prime}$ is the conjugate index. Of course the inequality (4.5) is equivalent to the bound

$$
\left\|H_{b}\right\|_{\mathcal{H}^{p} \rightarrow\left(\mathcal{H}^{q}\right)^{*}} \leq M\left(p, p e^{2 t / c}\right) M\left(q, q e^{2 t / c}\right)\left\|e^{t A} b\right\|_{r}
$$

where $\left(\mathcal{H}^{q}\right)^{*}$ is the dual space to $\mathcal{H}^{q}$. In general the space $\mathcal{H}^{q^{\prime}}$ may not be the dual space to $\mathcal{H}^{q}$. If $h \in \mathcal{H}^{q^{\prime}}$ then the linear functional $T_{h}: \mathcal{H}^{q} \rightarrow \mathbb{C}$ 
defined, as usual, by

$$
T_{h}(f)=\int_{M} f(z) \overline{h(z)} d \mu(z),
$$

has norm at most $\|h\|_{q^{\prime}}$ by Hölder's inequality. Since $\mathcal{H}^{q}$ is smaller than $L^{q}$ one might actually have $\left\|T_{h}\right\|_{\left(\mathcal{H}^{q}\right)^{*}}<\|h\|_{q^{\prime}}$. In fact it can actually happen that $T_{h}=0$ even if $h \neq 0$, as we will see in Example 4.19. If the map $h \mapsto T_{h}$ from $\mathcal{H}^{q^{\prime}}$ into $\left(\mathcal{H}^{q}\right)^{*}$ is one to one and onto then we will say that $\mathcal{H}^{q^{\prime}}$ is the full dual space to $\mathcal{H}^{q}$.

By the open mapping theorem $T_{(\cdot)}$ has a bounded inverse when $\mathcal{H}^{q^{\prime}}$ is the full dual space to $\mathcal{H}^{q}$. We summarize this as a proposition.

Proposition 4.17. Let $1<q<\infty$. If $\mathcal{H}^{q^{\prime}}$ is the full dual space, $\left(\mathcal{H}^{q}\right)^{*}$, then there exists a real constant $C_{q}>0$ such that

$$
C_{q}^{-1}\|h\|_{q^{\prime}} \leq\left\|T_{h}\right\|_{\left(\mathcal{H}^{q}\right)^{*}} \leq\|h\|_{q^{\prime}} \quad \forall h \in \mathcal{H}^{q^{\prime}} .
$$

Moreover $\mathcal{H}^{q}$ is also the full dual space to $\mathcal{H}^{q^{\prime}}$ and (4.29) holds with $q$ and $q^{\prime}$ interchanged, for some constant $C_{q^{\prime}}>0$.

The last assertion follows from the fact that the pairing (4.28) is nondegenerate when $\mathcal{H}^{q^{\prime}}$ is the full dual space to $\mathcal{H}^{q}$ and from the fact that $\mathcal{H}^{q}$ is reflexive, being a closed subspace of the reflexive space $L^{q}$.

When $\mathcal{H}^{q^{\prime}}$ is the full dual space to $\mathcal{H}^{q}$ the inequality (4.5) assures that for fixed $g \in \mathcal{H}^{p}$ there exists a unique element $H_{b} g \in \mathcal{H}^{q^{\prime}}$ such that $\Gamma_{b}(f, g)=$ $\left\langle f, H_{b} g\right\rangle$, and this defines the anti-linear Hankel operator $H_{b}: \mathcal{H}^{p} \rightarrow \mathcal{H}^{q^{\prime}}$ associated to the Hankel form $\Gamma_{b}$.

Corollary $4.18\left(H_{b}: \mathcal{H}^{p} \rightarrow \mathcal{H}^{q^{\prime}}\right.$ is bounded). Let $1<q<\infty$ and assume that $\mathcal{H}^{q}$ is the full dual space to $\mathcal{H}^{q^{\prime}}$. Then, under the hypothesis of Theorem 4.2 , one has

$$
\left\|H_{b}\right\|_{\mathcal{H}^{p} \rightarrow \mathcal{H}^{q^{\prime}}} \leq C_{q^{\prime}} M\left(p, p e^{2 t / c}\right) M\left(q, q e^{2 t / c}\right)\left\|e^{t A} b\right\|_{r}
$$

where $C_{q^{\prime}}$ is given by (4.29).

Proof. This follows from (4.27) and (4.29) (with $q$ replaced by $q^{\prime}$ ).

The duality assumption in Corollary 4.18 frequently fails. In Example 4.20 we will show that it even fails for Gauss measure. But first we will show in the following example that the converse of Proposition 4.17 is false. We will exploit the pathological Example 3.18 for this purpose.

Example 4.19. The converse of Proposition 4.17 is false. Choose $\lambda$ in Example 3.18 so large that $\operatorname{dim} \mathcal{H}^{p} \geq 2$ for some $p>1$. Clearly one can then choose $p$ so close to one, and therefore $p^{\prime}$ so large, that $\mathcal{H}^{p^{\prime}}$ consists only of constants. In this case $\operatorname{dim} \mathcal{H}^{p^{\prime}}=1<\operatorname{dim} \mathcal{H}^{p}$. So $\mathcal{H}^{p^{\prime}}$ cannot give the full dual space to $\mathcal{H}^{p}$. Yet (4.29) holds because $T_{1}(1) \neq 0$, which implies 
$\left\|T_{1}\right\|_{\left(\mathcal{H}^{p}\right)^{*}}>0$, which gives (4.29) for $h=1$, and therefore for all constant functions $h$. So (4.29) holds even though $\mathcal{H}^{p^{\prime}}$ is not the full dual space to $\mathcal{H}^{p}$.

The same example illustrates that the map $h \mapsto T_{h}$ need not be one to one: With the same $\lambda$ and $p$ as above take $q=p^{\prime}$. Then $\operatorname{dim} \mathcal{H}^{q^{\prime}}>1=\operatorname{dim}$ $\mathcal{H}^{q}$. So there exists a nonzero $h \in \mathcal{H}^{q^{\prime}}$ such that $T_{h}=0$.

P. Sjögren, $[\mathbf{S j}]$, has shown that $\mathcal{H}^{q^{\prime}}\left(\gamma_{a}\right)$ is not the full dual space to $\mathcal{H}^{q}\left(\gamma_{a}\right)$ for Gauss measure, $\gamma_{a}$, when $q \neq 2$. Another proof of Sjögren's theorem was given in [JPR, Corollary 9.2]. At the same time [JPR] identified the actual dual spaces. They showed, for example, that if $1<p<\infty$ then the dual space to $\mathcal{H} L^{p}\left(\gamma_{2 a / p}\right)$ is $\mathcal{H} L^{p^{\prime}}\left(\gamma_{2 a / p^{\prime}}\right)$ in the $L^{2}\left(\gamma_{a}\right)$ pairing.

In the following example we will give yet another proof of Sjögren's theorem, based on recent work of E. Carlen, $[\mathbf{C}]$.

Example 4.20 (Duality fails for Gauss measure on $\mathbb{C}^{m},[\mathbf{S} \mathbf{j}]$ ). Let $a>0$ and let $\gamma_{a}$ be the Gaussian measure on $\mathbb{C}^{m} \equiv \mathbb{R}^{2 m}$ with density given by (2.4). If $1<p<\infty$ but $p \neq 2$ then $\mathcal{H} L^{p^{\prime}}\left(\gamma_{a}\right)$ is not the full dual space to $\mathcal{H} L^{p}\left(\gamma_{a}\right)$.

Proof. It suffices to show that there is no constant $C_{p}>0$ such that (4.29) holds. Let $h_{z}(w):=e^{w \cdot z / 2 a}$. It is straightforward to compute that

$$
\left\|h_{z}\right\|_{L^{q}\left(\gamma_{a}\right)}=e^{q|z|^{2} / 8 a}, \quad 1 \leq q<\infty,
$$

since the integral is just a Laplace transform of $\gamma_{a}$. But $h_{z}$ is the reproducing kernel for $\mathcal{H} L^{2}\left(\gamma_{a}\right)$ [Ba]. So for all $f$ in $\mathcal{H} L^{2}\left(\gamma_{a}\right)$ we have

$$
\int_{\mathbb{C}^{m}} f(w) \bar{h}_{z}(w) d \mu=f(z) .
$$

If $f \in \mathcal{H} L^{p}\left(\gamma_{a}\right)$ then this equation holds by restriction if $p>2$ and by continuity of both sides in $\mathcal{H} L^{p}$ norm if $1<p<2$, since $\mathcal{H} L^{2}$ is dense in $\mathcal{H} L^{p}$ in this case. Now E. Carlen has computed explicitly the $\mathcal{H} L^{p}\left(\gamma_{a}\right)^{*}$ norm of the evaluation functional $\Lambda_{z}: f \mapsto f(z)$. He finds, [C, Equation I.22],

$$
\left\|\Lambda_{z}\right\|_{\mathcal{H} L^{p}\left(\gamma_{a}\right)^{*}}=e^{|z|^{2} / 2 p a}, \quad 1 \leq p<\infty .
$$

Since $T_{h_{z}}=\Lambda_{z}$ we therefore have

$$
\left\|T_{h_{z}}\right\|_{\mathcal{H} L^{p}\left(\gamma_{a}\right)^{*}} /\left\|h_{z}\right\|_{L^{p^{\prime}}\left(\gamma_{a}\right)}=e^{\left(1 / p-p^{\prime} / 4\right)|z|^{2} / 2 a} .
$$

But $p^{\prime} / 4-1 / p=(4 p)^{-1}\left(\frac{p^{2}}{p-1}-4\right)=(4 p)^{-1}(p-2)^{2} /(p-1)$. It follows that the coefficient of $|z|^{2} / 2 a$ in the right side of (4.32) is strictly negative if $p \in(1, \infty)$ and $p \neq 2$. Therefore the ratio on the left side of (4.32) goes to zero as $|z| \rightarrow \infty$. Hence the first inequality in (4.29) cannot hold (with $q=p$ ) for all of the functions $h_{z}$ if $C_{p}>0$. 


\section{The spectral algebra $\mathcal{R}$ and the Hilbert-Schmidt class.}

We will again assume that Standard Conditions hold (Notation 2.3) and that $\mathcal{R}$ is an algebra (Notation 2.9).

In all the examples that we have the operator $A \mid \mathcal{H}^{2}$ has a discrete spectrum with finite multiplicites and contains the constants in its domain. In all the interesting examples $\mathcal{H}^{2}$ has then the following structure, which will allow an illuminating and computable analysis of the Hilbert-Schmidt properties of Hankel operators.

Notation 5.1. Denote by $S$ the spectrum of $A \mid \mathcal{H}^{2}$ and by $E_{\lambda}$ the eigenspace of $A \mid \mathcal{H}^{2}$ for eigenvalue $\lambda$. Then:

(i) We may write $S:=\left\{\lambda_{0}, \lambda_{1}, \ldots\right\}$ with $0=\lambda_{0}<\lambda_{1}<\lambda_{2}<\ldots$ and

$$
\mathcal{H}^{2}=\oplus_{\lambda \in S} E_{\lambda} .
$$

(ii) $E_{0}=\{$ constants $\}$ and $\operatorname{dim} E_{\lambda}<\infty$ for all $\lambda \in S$.

(iii) If $f \in E_{\lambda}$ and $g \in E_{\nu}$ then $f g \in E_{\lambda+\nu}$. In particular $\lambda, \nu \in S$ implies $\lambda+\nu \in S$.

(iv) $\mathcal{R}$ is the linear (not closed) span of $\left\{E_{\lambda} \mid \lambda \in S\right\}$. Moreover $\mathcal{R} \subset$ $L^{p} \quad \forall p<\infty$.

Remark 5.2. Items (i) and (ii) just reflect the assumption that $A \mid \mathcal{H}^{2}$ has discrete spectrum with finite multiplicity and that the constants are in $D(Q)$. Clearly $Q(f)=0$ only for the constant functions. Item (i) and the assertion $\operatorname{dim} E_{\lambda}<\infty$ would both follow from a knowledge that $A$ has compact resolvent in $L^{2}$. In fact $A$ does indeed have compact resolvent if the logarithmic Sobolev inequality (2.21) holds, by the Bogachev-Rockner-Zhang-CiprianiWang theorem [BRZ, Ci, Wa]. But we do not need to assume that (2.21) holds elsewhere in this section and in fact (i) and (ii) hold quite a bit more generally. In the important case of the weighted Bergman spaces $\mathcal{B}_{\gamma}$, with $-1<\gamma<0$, (cf. Example 3.12) the validity of (i) and (ii) actually requires the use of non-Dirichlet boundary conditions on $A$, as we discussed in Examples 3.11 and 3.12. But since we just wish to show how the structure exhibited in items (i) to (iv) impinge on the Hilbert-Schmidt properties of Hankel operators we will not elaborate here on the boundary conditions which validate items (i) to (iv) for the weighted Bergman spaces.

Item (iv) just restates the definition of $\mathcal{R}$ in the present notation and item (iii) follows from the equation, cf. (2.15), $A(f g)=Z(f g)=(Z f) g+f(Z g)=$ $(\lambda+\nu) f g$. The validity of this equation hinges on our assumption, used throughout this section, that $\mathcal{R}$ is closed under multiplication and, of course, that Standard Conditions hold. All of the examples of Section 3 have the structure listed in items (i) to (iv) except for the pathological Example 3.18.

Our computations in this section will depend only on the properties (i) to (iv) of the space $\mathcal{H}^{2}$. For any function $f$ in $\mathcal{H}^{2}$ we will write $f=\sum_{\lambda} f_{\lambda}$ for 
the decomposition corresponding to the direct sum in Equation (5.1), and we will write simply $\|\cdot\|$ for $\|\cdot\|_{L^{2}(\mu)}$.

The map $\mathcal{H}^{2} \ni b \mapsto \Gamma_{b}$ is one to one because if $\langle f g, b\rangle=\Gamma_{b}(f, g)=0$ for all $f, g \in \mathcal{R}$ then $b=0$, since $\mathcal{R} \cdot \mathcal{R}=\mathcal{R}$, which is dense in $\mathcal{H}^{2}$. Thus the map $b \mapsto H_{b}$ is also one to one on the set of those $b \in \mathcal{H}^{2}$ for which $H_{b}$ exists as a bounded operator on $\mathcal{H}^{2}$. We are going to pull back the Hilbert-Schmidt (HS) norm on $H_{b}$ to $b$.

Notation 5.3. Let

$$
\mathcal{C}=\left\{b \in \mathcal{H}^{2}: H_{b} \text { is } \mathrm{HS}\right\}
$$

Define

$$
(b, c)_{\mathrm{HS}}=\operatorname{trace}_{\mathcal{H}^{2}}\left(H_{c}^{*} H_{b}\right) \text { for } b \text { and } c \in \mathcal{C} .
$$

Since the map $b \mapsto H_{b}$ is one to one $(,)_{\text {HS }}$ is an inner product on $\mathcal{C}$.

Theorem 5.4 (Structure of $\mathcal{C}$ ).

(a) $\mathcal{R} \subset \mathcal{C}$ and $\mathcal{C}$ is dense in $\mathcal{H}^{2}$.

(b) $\mathcal{C}$ is complete in the HS inner product, (5.3).

(c) $\mathcal{C}=\oplus_{\lambda \in S} E_{\lambda}$ in the HS inner product.

The proof of Theorem 5.4 depends on the following two lemmas.

Lemma 5.5. Let $\lambda \in S$. Suppose that $b \in E_{\lambda}$. Then $\Gamma_{b}$ is continuous on $\mathcal{R} \times \mathcal{R}$. The Hankel operator $H_{b}$ satisfies:

(a) $H_{b}\left(E_{\nu}\right) \subset E_{\lambda-\nu}$ if $\lambda-\nu \in S$.

(b) $H_{b}\left(E_{\nu}\right)=\{0\}$ if $\lambda-\nu \notin S$.

(c) range $\left(H_{b}\right) \subset \operatorname{span}\left\{E_{\sigma}: \sigma \leq \lambda\right\}$. So $H_{b}$ is a continuous finite rank operator with

$$
\operatorname{rank}\left(H_{b}\right) \leq \sum_{\sigma \leq \lambda} \operatorname{dim}\left(E_{\sigma}\right)
$$

In particular $H_{b}$ is of $\mathrm{HS}$ type.

(d) If $b \in E_{\lambda}$ and $c \in E_{\sigma}$ and $\lambda \neq \sigma$ then

$$
\operatorname{trace}_{\mathcal{H}^{2}}\left(H_{c}^{*} H_{b}\right)=0 .
$$

(e) $\|b\| \leq\|b\|_{\mathrm{HS}}$ for all $b \in \mathcal{C}$.

Proof. Suppose that $b \in E_{\lambda}$. Let $F_{\lambda}=\operatorname{span}\left\{E_{\sigma}: \sigma \leq \lambda\right\}$. Then $F_{\lambda}$ is a finite dimensional subspace of $\mathcal{R}$, which is itself contained in $L^{4}$. Hence the $L^{4}$ and $L^{2}$ norms are equivalent on $F_{\lambda}$. Thus if $P_{\lambda}: \mathcal{H}^{2} \rightarrow F_{\lambda}$ is the orthogonal projection then for any $g \in \mathcal{H}^{2}$ one has $\left\|\overline{\left(P_{\lambda} g\right)} b\right\|_{2} \leq\left\|P_{\lambda} g\right\|_{4}\|b\|_{4} \leq$ $C_{\lambda}\left\|P_{\lambda} g\right\|_{2}\|b\|_{4} \leq C_{\lambda}\|g\|_{2}\|b\|_{4}$ for some constant $C_{\lambda}$. So the operator

$$
H: g \mapsto P_{\mathcal{H}^{2}}\left[\overline{\left(P_{\lambda} g\right)} b\right]
$$


is continuous from $\mathcal{H}^{2}$ to $\mathcal{H}^{2}$ with norm at most $C_{\lambda}\|b\|_{4}$. Now suppose that $g \in E_{\nu}$. If $\nu \leq \lambda$ then $g=P_{\lambda} g$. So $\Gamma_{b}(f, g)=\langle f, \bar{g} b\rangle=\left\langle f, \overline{\left(P_{\lambda} g\right)} b\right\rangle=$ $\langle f, H g\rangle$ for all $f \in \mathcal{R}$. If $\nu>\lambda$ then $P_{\lambda} g=0$. So $\langle f, H g\rangle=0$. But also $\Gamma_{b}(f, g)=\langle f g, b\rangle=0$ when $f \in E_{\sigma}$ because $f g \in E_{\nu+\sigma}$ and $\sigma+\nu>\lambda$. Hence $\langle f, H g\rangle=0=\Gamma_{b}(f, g)$ for all $f \in \mathcal{R}$ when $\nu>\lambda$. So $\Gamma_{b}(f, g)=\langle f, H g\rangle$ for all $f, g \in \mathcal{R}$. Hence $\Gamma_{b}$ is continuous on $\mathcal{R} \times \mathcal{R}$ and $H_{b}=H$. Since $\left\langle f, H_{b} g\right\rangle=\Gamma_{b}(f, g)=0$ if $\sigma+\nu \neq \lambda$ the conclusions (a), (b) and (c) now follow. To prove (d) suppose that $c \in E_{\sigma}$ with $\sigma \neq \lambda$. If $g \in E_{\nu}$ then $\left\langle H_{c}^{*} H_{b} g, g\right\rangle=\left\langle H_{b} g, H_{c} g\right\rangle=0$ because $H_{b} g \in E_{\lambda-\nu}$ and $H_{c} g \in E_{\sigma-\nu}$. Thus if one computes the trace of $H_{c}^{*} H_{b}$ on a basis adapted to the decomposition (5.1) all terms are zero. Finally (e) follows by observing that $H_{b} 1=b$ for all $b \in \mathcal{C}$. Computing the trace of $H_{b}^{*} H_{b}$ in a basis in which the first term is the constant function equal to one we then find $\left\|H_{b}\right\|_{\mathrm{HS}}^{2} \geq\left\langle H_{b} 1, H_{b} 1\right\rangle=\langle b, b\rangle$, which gives (e).

Lemma 5.6. Suppose that $b=\sum_{\lambda \in S} b_{\lambda}$ is in $\mathcal{C}$ and that $c \in E_{\sigma}$. Then

$$
\operatorname{trace}_{\mathcal{H}^{2}}\left(H_{c}^{*} H_{b}\right)=\operatorname{trace}_{\mathcal{H}^{2}}\left(H_{c}^{*} H_{b_{\sigma}}\right) \text {. }
$$

Proof. Let $g \in E_{\nu}$. Then $f \equiv H_{c} g \in E_{\sigma-\nu}$ which should be interpreted to be $\{0\}$ if $\sigma-\nu \notin S$. So $f g \in E_{\sigma}$. Hence $\left\langle H_{c} g, H_{b} g\right\rangle=\left\langle f, H_{b} g\right\rangle=\langle f g, b\rangle=$ $\left\langle f g, b_{\sigma}\right\rangle=\left\langle H_{c} g, H_{b_{\sigma}} g\right\rangle$. So $\left\langle H_{b} g, H_{c} g\right\rangle=\left\langle H_{b_{\sigma}} g, H_{c} g\right\rangle$ for $g \in E_{\nu}$. Summing over a basis adapted to the decomposition in Equation (5.1) completes the proof.

Proof of Theorem 5.4. Lemma 5.5 shows that $E_{\lambda} \subset \mathcal{C}$ for all $\lambda \in S$. This proves (a).

To prove (b) assume that $b_{n}$ is a Cauchy sequence in $\mathcal{C}$ in HS norm. By Lemma 5.5, Part (e), it is also a Cauchy sequence in $\mathcal{H}^{2}$ norm. So there exists $b \in \mathcal{H}^{2}$ such that $\left\|b-b_{n}\right\| \rightarrow 0$. We must show that $b \in \mathcal{C}$ and that $\left\|b-b_{n}\right\|_{\mathrm{HS}} \rightarrow 0$. Since the space of HS operators on $\mathcal{H}^{2}$ is complete there exists a HS operator $H$ such that $\left\|H-H_{b_{n}}\right\|_{\text {HS }} \rightarrow 0$. So for $f$ and $g$ in $\mathcal{R}$ one has $\langle f, H g\rangle=\lim _{n \rightarrow \infty}\left\langle f, H_{b_{n}} g\right\rangle=\lim _{n \rightarrow \infty}\left\langle f g, b_{n}\right\rangle=\langle f g, b\rangle$ because $f g \in \mathcal{H}^{2}$. Hence $\Gamma_{b}$ is continuous and $H_{b}=H$. So $b \in \mathcal{C}$ and $\left\|H_{b}-H_{b_{n}}\right\|_{\mathrm{HS}} \rightarrow 0$.

To prove (c) observe that by Lemma 5.5, Part (d), we have $E_{\lambda} \perp E_{\sigma}$ in HS inner product if $\lambda \neq \sigma$. The sum in item (c) of Theorem 5.4 is therefore an orthogonal sum in HS inner product. It suffices then to show that if $b \in \mathcal{C}$ and if $(b, c)_{\mathrm{HS}}=0$ for all $c \in E_{\sigma}$ and all $\sigma \in S$ then $b=0$. But if $b=\sum_{\lambda} b_{\lambda}$ then Lemma 5.6 shows that $0=\left(b, b_{\sigma}\right)_{\mathrm{HS}}=\left(b_{\sigma}, b_{\sigma}\right)_{\mathrm{HS}}$. Hence $b_{\sigma}=0$ for all $\sigma \in S$ and therefore $b=0$.

Corollary 5.7. If $b=\sum_{\lambda \in S} b_{\lambda} \in \mathcal{H}^{2}$ then

$$
\left\|H_{b}\right\|_{\mathrm{HS}}^{2}=\sum_{\lambda \in S}\left\|H_{b_{\lambda}}\right\|_{\mathrm{HS}}^{2} .
$$


If either side is finite then both sides are finite and $H_{b}$ admits the $\mathrm{HS}$ orthogonal decomposition

$$
H_{b}=\sum_{\lambda} H_{b_{\lambda}}
$$

(with convergence in HS norm).

Proof. By (5.3) the linear map $b \mapsto H_{b}$ is isometric from the Hilbert space $\left(\mathcal{C},\|\cdot\|_{\mathrm{HS}}\right)$ into the HS operators on $\mathcal{H}^{2}$. Thus by Theorem $5.4(\mathrm{c}) H_{b}=$ $\sum_{\lambda} H_{b_{\lambda}}$ is an orthogonal decomposition corresponding to $b=\sum_{\lambda} b_{\lambda}$ under this isometry, and (5.6) follows. Clearly both sides in (5.6) are infinite if $b \notin \mathcal{C}$.

Four computable examples in one complex dimension. In each of the following examples $E_{\lambda}$ will be one dimensional and the spectrum $S$ will have the form $S=\{n a: n=0,1,2, \ldots\}$ for some constant $a>0$. $M$ will be chosen to be either:

(1) $\mathbb{C}-\{0\}$ (Example 5.11),

(2) the Riemann surface for $z^{1 / m}$ with $m \geq 2$ (Example 5.12),

(3) $\mathbb{C}$ (Example 5.12 with $m=1$ ), or

(4) the unit disk (Example 5.13).

We choose an element $\varphi_{n} \in E_{n a}$ for each $n \geq 0$ in such a way that $\varphi_{n} \varphi_{m}=\varphi_{n+m}$ for all $n, m \geq 0$. For example we will take $\varphi_{n}(z)=z^{n}$ when $M$ is a subset of $\mathbb{C}$ and we will take $\varphi_{n}(z)=z^{n / m}$ in the case of the Riemann surface for $z^{1 / m}$. The following lemma is the basis for our computations of HS norms of Hankel operators.

Lemma 5.8. Suppose $\varphi_{n}$ spans $E_{n a}$ for $n \geq 0$ as in the immediately preceding notation and $\varphi_{n} \varphi_{m}=\varphi_{n+m}$ for all $n, m \geq 0$. Then

$$
\left\|H_{\varphi_{n}}\right\|_{\mathrm{HS}}^{2}=\sum_{k=0}^{n} \frac{\left\|\varphi_{n}\right\|^{4}}{\left\|\varphi_{k}\right\|^{2}\left\|\varphi_{n-k}\right\|^{2}}
$$

Proof. By Lemma 5.5, Parts (a) and (b), we know that $H_{\varphi_{n}} \varphi_{k}=a_{n k} \varphi_{n-k}$ for $n \geq k$, and $H_{\varphi_{n}} \varphi_{k}=0$ for $n<k$. The constants $a_{n k}$ follow from

$$
a_{n k}\left\|\varphi_{n-k}\right\|^{2}=\left\langle H_{\varphi_{n}} \varphi_{k}, \varphi_{n-k}\right\rangle=\left\langle\varphi_{n}, \varphi_{k} \varphi_{n-k}\right\rangle=\left\|\varphi_{n}\right\|^{2},
$$

which gives $a_{n k}=\left\|\varphi_{n}\right\|^{2} /\left\|\varphi_{n-k}\right\|^{2}$. This yields

$$
\left\|H_{\varphi_{n}}\right\|_{\mathrm{HS}}^{2}=\sum_{k=0}^{\infty}\left\|H_{\varphi_{n}} \frac{\varphi_{k}}{\left\|\varphi_{k}\right\|}\right\|^{2}=\sum_{k=0}^{n} \frac{a_{n k}^{2}\left\|\varphi_{n-k}\right\|^{2}}{\left\|\varphi_{k}\right\|^{2}}=\sum_{k=0}^{n} \frac{\left\|\varphi_{n}\right\|^{4}\left\|\varphi_{n-k}\right\|^{2}}{\left\|\varphi_{n-k}\right\|^{4}\left\|\varphi_{k}\right\|^{2}} .
$$

Lemma 5.9. Continuing the notation of the preceding lemma, suppose that there is another measure $\widetilde{\mu}$ on $M$ such that the $\varphi_{n}$ are mutually orthogonal in $L^{2}(\widetilde{\mu})$ and there are constants $0<r \leq s<\infty$ with

$$
r\left\|\varphi_{n}\right\|_{L^{2}(\widetilde{\mu})}^{2} \leq\left\|H_{\varphi_{n}}\right\|_{\mathrm{HS}}^{2} \leq s\left\|\varphi_{n}\right\|_{L^{2}(\widetilde{\mu})}^{2}, \text { for } n=0,1,2, \ldots
$$




\section{Then}

$$
r\|b\|_{L^{2}(\widetilde{\mu})}^{2} \leq\left\|H_{b}\right\|_{\mathrm{HS}}^{2} \leq s\|b\|_{L^{2}(\widetilde{\mu})}^{2} \forall b \in \mathcal{H}^{2} .
$$

Proof. If $b=\sum_{n=0}^{\infty} \alpha_{n} \varphi_{n}$ then, in view of Corollary 5.7, the inequality (5.9) follows from (5.8) by multiplying (5.8) by $\left|\alpha_{n}\right|^{2}$ and summing over $n$.

Remark 5.10. It is known, [JPR, Theorem 7.8], that in the Gaussian case, with $\mu=\gamma_{a}$, one has $(b, b)_{\mathrm{HS}}=\|b\|_{L^{2}\left(\gamma_{2 a}\right)}^{2}$. That is, (5.9) holds with $r=s=1$ and $\widetilde{\mu}=\gamma_{2 a}$. We will compute the norms $\|b\|_{\text {HS }}$ in four examples using Lemma 5.8 and show that in those three examples in which the flow of $X$ is two-sided complete the inequalities (5.9) hold with $\widetilde{\mu}=\left(e^{t X}\right)_{*} \mu$ for a suitable $t>0$ or (in Example 5.11) a slight variation of this measure. In contrast to these three cases we will show that (5.8) cannot hold for the weighted Bergman spaces for any finite measure on the unit disk. The $X$ flow is not two-sided complete for the weighted Bergman spaces. These four examples suggest that there may be a link between the validity of an equivalence of norms as in (5.9) and the two-sided completeness of the $X$ flow, at least when a logarithmic Sobolev inequality, (2.21), is present. But we have not explored this possibility.

Example 5.11. We take $M=\mathbb{C}-\{0\}$ as in Example 3.8. Let $d \nu_{a}(z)$ be the measure on $M$ with density given by (3.19). One can compute easily that $\left\|z^{n}\right\|_{L^{2}\left(\nu_{a}\right)}^{2}=a^{2 n}(2 n+1)$ !. Then by Lemma $5.8\left\|H_{z^{n}}\right\|_{\mathrm{HS}\left(\nu_{a}\right)}^{2}=a^{2 n}(2 n+$ $1) ! \sum_{k=0}^{n} \frac{1}{2 k+1}\left(\begin{array}{c}2 n+1 \\ 2 k\end{array}\right)=a^{2 n}(2 n+1) ! \frac{2^{2 n}}{n+1}$. (The sum follows from formulas 0.155 .1 and 0.155 .2 in $[\mathbf{G r R}]$; Maple $\mathrm{V}$ gives the same result.) With $N=$ $z \partial / \partial z$ we see that

$$
(N+1)^{-1 / 2} 2^{N} z^{n}=\frac{2^{n}}{(1+n)^{1 / 2}} z^{n} .
$$

Let $b=\sum_{n=0}^{\infty} c_{n} z^{n}$. Equation (5.6) shows that

$$
\begin{aligned}
\left\|H_{b}\right\|_{\mathrm{HS}}^{2} & =\sum_{n=0}^{\infty}\left|c_{n}\right|^{2} a^{2 n}(2 n+1) ! \frac{2^{2 n}}{n+1} \\
& =\sum_{n=0}^{\infty}\left|c_{n}\right|^{2} \frac{2^{2 n}}{n+1}\left\|z^{n}\right\|_{L^{2}\left(\nu_{a}\right)}^{2}=\left\|(1+N)^{-1 / 2} 2^{N} b\right\|_{L^{2}\left(\nu_{a}\right)}^{2} .
\end{aligned}
$$

Thus

$$
\left\|H_{b}\right\|_{\mathrm{HS}}^{2}=\left\|2^{N}(1+N)^{-1 / 2} b\right\|_{L^{2}\left(\nu_{a}\right)}^{2} .
$$

In accordance with Proposition 4.14 and Equation (4.22) we may write this as

$$
\left\|H_{b}\right\|_{\operatorname{HS}\left(\mathcal{H}^{2}\left(\nu_{a}\right)\right)}^{2}=\left\|(1+N)^{-1 / 2} b\right\|_{L^{2}\left(\nu_{2 a}\right)} .
$$


as we see by writing $2^{N}=e^{(\log 2)(A / 2)}$ and taking $t=(1 / 2) \log 2$ and $n=2$ in (4.22). Because of the factor $(1+N)^{-1 / 2}$ the right side of $(5.11)$ does not represent a pure growth condition on $b$ in the spirit of (5.9). But we can convert it into such a condition as follows. Let

$$
d \widetilde{\nu}_{a}(z):=\frac{1}{2 \pi a} \frac{e^{-|z| / a}}{|z|} .
$$

One has then $\left\|z^{n}\right\|_{L^{2}\left(\widetilde{\nu}_{a}\right)}^{2}=a^{2 n}(2 n)$ !. So

$$
\begin{aligned}
\left\|H_{b}\right\|_{\mathrm{HS}\left(\mathcal{H}^{2}\left(\nu_{a}\right)\right)}^{2} & =\sum_{n=0}^{\infty}\left|c_{n}\right|^{2} a^{2 n}(2 n+1) ! \frac{2^{2 n}}{n+1} \\
& =\sum_{n=0}^{\infty}\left|c_{n}\right|^{2}\left\|z^{n}\right\|_{L^{2}\left(\widetilde{\nu}_{2 a}\right)}^{2} \frac{2 n+1}{n+1} .
\end{aligned}
$$

Hence

$$
\|b\|_{L^{2}\left(\widetilde{\nu}_{2 a}\right)}^{2} \leq\left\|H_{b}\right\|_{\operatorname{HS}\left(\mathcal{H}^{2}\left(\nu_{a}\right)\right)}^{2} \leq 2\|b\|_{L^{2}\left(\widetilde{\nu}_{2 a}\right)}^{2} .
$$

Thus if $b \in \mathcal{H}^{2}\left(\mathbb{C}_{*}, \nu_{a}\right)$ then (5.9) holds with $\widetilde{\mu}=\widetilde{\nu}_{2 a}$ and with $r=1$ and $s=2$. Our derivation of (5.13) from (5.11) is somewhat ad hoc. Here is a more conceptual, though longer, derivation which may be useful for future extensions. Consider the lift $\mu_{a}$ of $\nu_{a}$ to $M_{2}$ as in Examples 3.9 and 3.10. Notice that $\mathcal{H}^{2}\left(\mathbb{C}_{*}, \nu_{a}\right)$ is naturally embedded in $\mathcal{H}^{2}\left(M_{2}, \mu_{a}\right)$ by regarding it as the subspace of functions on $M_{2}$ which are invariant under the interchange of the two leaves of $M_{2}$ (and so spanned by the functions $z^{k / 2}$ with $k$ even and nonnegative). Extend the operator denoted above by $N$ to $\mathcal{H}^{2}\left(M_{2}, \mu_{a}\right)$ by putting $N=z \partial / \partial z$ in $\mathcal{H}^{2}\left(M_{2}, \mu_{a}\right)$. Since $A f=Z f=2 z f^{\prime}(z)$ over $M_{2}$ we have $A=2 N$. By (3.23) with $n=2$ we therefore have $C^{*} C=2 a(N+1)$. Recall that $C$ is multiplication by $z^{1 / 2}$, which makes sense over $M_{2}$ but not over $\mathbb{C}_{*}$. Let $d \widetilde{\mu}_{a}=(a /|z|) d \mu_{a}$ on $M_{2}$. The key observation in the following is that the creation operator relates the two probability measures thus: $\left\|z^{1 / 2} g\right\|_{\widetilde{\mu}_{a}}^{2}=a\|g\|_{\mu_{a}}^{2}$. Now any function $f \in \mathcal{R}$ over $M_{2}$ may be written $f=\gamma+C g$ for some $\gamma \in \mathbb{C}$ and $g \in \mathcal{R}$. Then

$$
\|f\|_{\widetilde{\mu}_{a}}^{2}=|\gamma|^{2}+\|C g\|_{\widetilde{\mu}_{a}}^{2}=|\gamma|^{2}+a\|g\|_{\mu_{a}}^{2} .
$$

Also,

$$
\begin{aligned}
\left\langle(N+1)^{-1} f, f\right\rangle_{\mu_{a}} & =|\gamma|^{2}+\left\langle(N+1)^{-1} C g, C g\right\rangle_{\mu_{a}} \\
& =|\gamma|^{2}+\left\langle C^{*}(N+1)^{-1} C g, g\right\rangle_{\mu_{a}} .
\end{aligned}
$$

But from (3.24) with $n=2$ we have $A C=C(A+1)$. So $N C=C(N+1 / 2)$ and therefore $(N+1) C=C(N+3 / 2)$ on $\mathcal{R}$. Hence $C^{*}(N+1)^{-1} C=$ $C^{*} C(N+3 / 2)^{-1}=2 a(N+1)(N+3 / 2)^{-1}$ by (3.23). Consequently $a \leq$ 
$C^{*}(N+1)^{-1} C \leq 2 a$. Combining this with (5.14) and (5.15) gives

$$
\|f\|_{\widetilde{\mu}_{a}}^{2} \leq\left\|(N+1)^{-1 / 2} f\right\|_{\mu_{a}}^{2} \leq 2\|f\|_{\widetilde{\mu}_{a}}^{2}
$$

first for all $f \in \mathcal{R}$ and then for all $f \in \mathcal{H}^{2}\left(M_{2}, \mu_{a}\right)$. Replacing now $a$ by $2 a$ in (5.16) and choosing $f=b \in \mathcal{H}^{2}\left(\mathbb{C}_{*}, \nu_{2 a}\right)$ the inequality (5.16) combined with (5.11) gives (5.13) because $\widetilde{\mu}_{2 a}$ is the lift of $\widetilde{\nu}_{2 a}$ to $M_{2}$.

Example 5.12 (The Riemann surface for $z^{1 / m}$ and the Gaussian subcase). We take the notation from Example 3.4 but replace $n$ by $m$. We will prove that (5.8) holds for some $0<r<s<\infty$. Consider the orthogonal basis $\left\{\varphi_{n}(z):=z^{n / m}, n \in \mathbb{N}_{0}\right\}$ in the space $\mathcal{H}^{2}\left(M_{m}, \mu_{a}\right)$ over the Riemann Surface $M_{m}$ with $m \geq 2$ fixed, where we have written $\mu_{a}$ for the measure $\mu$ defined in Example 3.4. Then

$$
\left\|\varphi_{n}\right\|_{L^{2}\left(\mu_{a}\right)}^{2}=(2 a)^{n / m} \Gamma\left(\frac{n}{m}+1\right)
$$

and a simple calculation based on Lemma 5.8 yields

$$
\left\|H_{\varphi_{n}}\right\|_{\mathrm{HS}}^{2}=(2 a)^{n / m} \Gamma\left(\frac{n}{m}+1\right) \sum_{k=0}^{n} \frac{\Gamma\left(\frac{n}{m}+1\right)}{\Gamma\left(\frac{n-k}{m}+1\right) \Gamma\left(\frac{k}{m}+1\right)} .
$$

Gaussian subcase: For $m=1$ this formula is also correct for Gauss measure $\gamma_{a}$ on $\mathbb{C}$ and (5.18) then reduces to

$$
\left\|H_{\varphi_{n}}\right\|_{\mathrm{HS}}^{2}=(2 a)^{n} n ! \sum_{k=0}^{n}\left(\begin{array}{l}
n \\
k
\end{array}\right)=(4 a)^{n} n !=\left\|\varphi_{n}\right\|_{L^{2}\left(\gamma_{2 a}\right)}^{2} .
$$

So (5.9) holds for $\mathcal{H}^{2}\left(\mathbb{C}, \gamma_{a}\right), \widetilde{\gamma}_{a}=\gamma_{2 a}$ and $r=s=1$. This result has been obtained by different techniques in [JPR, Theorem 7.8]. Yet another derivation of (5.19) is given in Example 6.9.

For $m \geq 2$ we use the beta function $B(x, y):=\Gamma(x) \Gamma(y) / \Gamma(x+y)$ which satisfies

$$
\frac{1}{B(x, y)}=\frac{2^{x+y-1}(x+y-1)}{\pi} \int_{0}^{\frac{\pi}{2}} \cos [(x-y) t] \cos ^{x+y-2}(t) d t,
$$

see [GrR, p. 949]. With $x=\frac{n-k}{m}+1, y=\frac{k}{m}+1$ and $\Gamma(x+1)=x \Gamma(x)$ we write (5.18) as

$$
\left\|H_{\varphi_{n}}\right\|_{\mathrm{HS}}^{2}=\frac{2}{\pi}(4 a)^{n / m} \Gamma\left(\frac{n}{m}+1\right) \int_{0}^{\frac{\pi}{2}}\left[\sum_{k=0}^{n} \cos \left(\frac{n-2 k}{m} t\right)\right] \cos ^{n / m} t d t .
$$

Now (5.17) gives the relation with the norm of $\varphi_{n}$ in $L^{2}\left(\mu_{2 a}\right)$,

$$
\left\|H_{\varphi_{n}}\right\|_{\mathrm{HS}}^{2}=\frac{2}{\pi}\left\|\varphi_{n}\right\|_{L^{2}\left(\mu_{2 a}\right)}^{2} \cdot c_{n},
$$


where $c_{n}$ is the integral in (5.20). We can do the summation in (5.20) using the finite geometric series for complex exponentials. After elementary trigonometric manipulations we obtain

$$
c_{n}=\int_{0}^{\frac{\pi}{2}}\left(\cos \left(\frac{n}{m} t\right)+\frac{\sin \left(\frac{n}{m} t\right) \cos \frac{t}{m}}{\sin \frac{t}{m}}\right) \cos ^{n / m} t d t .
$$

Below we will prove that there exist constants $0<r<s$ and $n_{0} \in \mathbb{N}$ such that

$$
r \leq c_{n} \leq s \quad \forall n \geq n_{0} .
$$

With Lemma 5.9 and (5.21) we thus arrive at our final conclusion: Let $b \in \mathcal{H}^{2}\left(M_{m}, \mu_{a}\right)$. Then $b$ is the symbol of a HS-Hankel operator on $\mathcal{H}^{2}\left(\mu_{a}\right)$ if and only if $b \in \mathcal{H}^{2}\left(\mu_{2 a}\right)$. Notice that this characterization is identical with the one for $m=1$, but for $m \geq 2$ we do not obtain the norm equality (5.19). We only obtain norm equivalence, (5.9), by (5.21) and (5.22).

Proof of (5.22). Clearly $\int_{0}^{\frac{\pi}{2}} \cos \frac{n}{m} t \cos ^{\frac{n}{m}} t d t \rightarrow 0$ for $n \rightarrow \infty$, so it suffices to show that

$$
d_{n}:=\int_{0}^{\frac{\pi}{2 m}} \frac{\sin n x}{\sin x} \cos x \cos ^{\frac{n}{m}} m x d x
$$

is bounded from above and from below by strictly positive constants for $n \geq n_{0}$. We denote by $f_{n}(x)$ the integrand in (5.23).

Upper bound for $d_{n}$ : Let $k$ be the largest natural number satisfying $(2 k+$ 1) $\frac{\pi}{n} \leq \frac{\pi}{2 m}$. Then, for $n>2 m$,

$$
d_{n}=\int_{0}^{\frac{\pi}{n}} f_{n}(x) d x+\sum_{j=1}^{k} \int_{(2 j-1) \frac{\pi}{n}}^{(2 j+1) \frac{\pi}{n}} f_{n}(x) d x+r_{n} .
$$

Since $\sin n x$ is $\frac{2 \pi}{n}$-periodic and $\cot x \cos ^{\frac{n}{m}} m x$ decreases monotonicly on $\left[\frac{\pi}{n}, \frac{\pi}{2 m}\right]$ the terms in the sum in (5.24) are all non-positive, so

$$
d_{n} \leq \int_{0}^{\frac{\pi}{n}} f_{n}(x) d x+r_{n} \text { for all } n>2 m .
$$

Obviously $r_{n} \rightarrow 0$ for $n \rightarrow \infty$. The upper estimate therefore follows from

$$
\int_{0}^{\frac{\pi}{n}} f_{n}(x) d x \leq 2 \int_{0}^{\frac{\pi}{2 n}} \frac{\sin n x}{x / 2} d x<4 \int_{0}^{\frac{\pi}{2 n}} \frac{n x}{x} d x=2 \pi, \forall n>2 m .
$$


Lower bound for $d_{n}$ : For $x \in\left[0, \frac{\pi}{n}\right]$ and $n>4 m$ we have

$$
\begin{aligned}
\cos x \cos ^{\frac{n}{m}} m x & =\left(1-\frac{x^{2}}{2}+\ldots\right)\left(1-\frac{(m x)^{2}}{2}+\ldots\right)^{\frac{n}{m}} \\
& \geq\left(1-\frac{x^{2}}{2}\right)\left(1-\frac{(m x)^{2}}{2}\right)^{n} \\
& \geq\left(1-\frac{\pi^{2}}{2 n^{2}}\right)\left(1-\frac{m^{2} \pi^{2}}{2 n^{2}}\right)^{n}=: \alpha_{n} \rightarrow 1 \text { as } n \rightarrow \infty .
\end{aligned}
$$

Let $k$ be the largest natural number satisfying $(2 k+2) \frac{\pi}{n} \leq \frac{\pi}{2 m}$. For $n>4 m$ we estimate

$$
\begin{aligned}
d_{n} & =\int_{0}^{\frac{2 \pi}{n}} f_{n}(x) d x+\sum_{j=1}^{k} \int_{2 j \frac{\pi}{n}}^{(2 j+2) \frac{\pi}{n}} f_{n}(x) d x+\widetilde{r}_{n} \\
& \geq \alpha_{n} \int_{0}^{\frac{\pi}{n}} \frac{\sin n x}{\sin x} d x+\int_{\frac{\pi}{n}}^{\frac{2 \pi}{n}} \frac{\sin n x}{\sin x} d x+\widetilde{r}_{n} \\
& \geq \alpha_{n} \int_{0}^{\frac{\pi}{n}} \frac{\sin n x}{x} d x+\int_{\frac{\pi}{n}}^{\frac{2 \pi}{n}} \frac{\sin n x}{\sin \frac{\pi}{n}} d x+\widetilde{r}_{n} \\
& \geq \alpha_{n} \int_{0}^{\frac{\pi}{2 n}} \frac{2 n}{\pi} d x-\frac{2}{n \sin \frac{\pi}{n}}+\widetilde{r}_{n} \rightarrow 1-\frac{2}{\pi} \text { as } n \rightarrow \infty .
\end{aligned}
$$

Example 5.13 (Weighted Bergman spaces $\mathcal{B}_{\gamma}, \gamma>-1$ ). Continuing the notation of Example 3.12, we will show that $H_{b}$ is HS if and only if $b \in$ $D\left(A^{(\gamma+2) / 2} \mid \mathcal{H}^{2}\right)$. We consider first the case $\gamma=0$ because $\left\|H_{z^{n}}\right\|_{\mathrm{HS}\left(\mu_{0}\right)}$ can then be calculated explicitly. We have $\left\|z^{n}\right\|_{L^{2}\left(\mu_{0}\right)}^{2}=\frac{1}{n+1}$. So Lemma 5.8 yields

$$
\left\|H_{z^{n}}\right\|_{\mathrm{HS}\left(\mu_{0}\right)}^{2}=\sum_{k=0}^{n} \frac{(k+1)(n-k+1)}{(n+1)^{2}}=\frac{1}{6} \frac{(n+2)(n+3)}{n+1} .
$$

With $b(z)=\sum_{n=0}^{\infty} \alpha_{n} z^{n}$, Equation (5.6) shows that

$$
\begin{aligned}
b \in \mathcal{B}_{0} \text { and } H_{b} \text { is HS } & \Longleftrightarrow \sum_{n=0}^{\infty}\left|\alpha_{n}\right|^{2} \frac{1}{6} \frac{(n+2)(n+3)}{n+1}<\infty \\
& \Longleftrightarrow \sum_{n=0}^{\infty}\left|\alpha_{n}\right|^{2}(n+1)<\infty \\
& \Longleftrightarrow \int_{|z|<1}\left|b^{\prime}(z)\right|^{2} d x d y<\infty .
\end{aligned}
$$


This last condition coincides with the definition of the Besov space $B_{2}$ on the unit disk $D$, see $\left[\mathbf{Z h}\right.$, Sec. 5.3]. Thus $H_{b}$ is HS iff $b \in B_{2}$, cf. [ZZh, Sec. 5.7, Cor. 2]. The same characterization is actually valid for all weights $\gamma>-1$, [Pe1], [JPR, Theorem 12.1], which can be deduced by our method as follows. Observe first that

$$
\left\|H_{z^{n}}\right\|_{\mathrm{HS}}^{2}=\sum_{k=0}^{n} \frac{\left\|z^{n}\right\|^{2}}{\left\|z^{n-k}\right\|^{2}} \frac{\left\|z^{n}\right\|^{2}}{\left\|z^{k}\right\|^{2}} \leq \sum_{k=0}^{n} 1=n+1,
$$

because the $\left\|z^{n}\right\|$ are monotonicly decreasing (for all probability measures $\mu$ on the unit disk $D$ ). On the other hand, writing $\gamma=m-1+\alpha$ with $m \in \mathbb{N}_{0}$ and $\alpha \in(0,1]$ yields

$$
\begin{aligned}
\left\|z^{n}\right\|_{L^{2}\left(\mu_{\gamma}\right)}^{2} & =\Gamma(2+\gamma) \frac{n !}{\Gamma(n+2+\gamma)} \\
& =\Gamma(2+\gamma) \frac{\Gamma(n+1)}{(n+\alpha+m) \ldots(n+\alpha+1) \Gamma(n+\alpha+1)},
\end{aligned}
$$

and the asymptotic expansion $\Gamma(x+1) \sim(x / e)^{x} \sqrt{2 \pi x}$ applied to the right side shows that $\left\|z^{n}\right\|_{L^{2}\left(\mu_{\gamma}\right)}^{2} \sim d \cdot(n+\alpha)^{-\alpha-m}$ with some constant $d>0$. This implies that for all $n \geq n_{0}$ we can estimate

$$
\begin{aligned}
\left\|H_{z^{n}}\right\|_{\mathrm{HS}\left(\mu_{\gamma}\right)}^{2} & \geq \frac{1}{2} \sum_{k=\left[\frac{n}{3}\right]}^{\left[\frac{2 n}{3}\right]} \frac{(n-k+\alpha)^{\alpha+m}(k+\alpha)^{\alpha+m}}{(n+\alpha)^{2(\alpha+m)}} \\
& \geq \frac{1}{2}\left\{\sum_{k=\left[\frac{n}{3}\right]}^{\left[\frac{2 n}{3}\right]}\left(1-\frac{k+1}{n+1}\right)^{\alpha+m}\left(\frac{k}{n+1}\right)^{\alpha+m} \frac{1}{n+1}\right\}(n+1) .
\end{aligned}
$$

Since the term in brackets converges to $\int_{1 / 3}^{2 / 3}(1-x)^{\alpha+m} x^{\alpha+m} d x>0$ as $n \rightarrow$ $\infty$ there exists $c>0$ such that $\left\|H_{z^{n}}\right\|_{\mathrm{HS}\left(\mu_{\gamma}\right)}^{2} \geq c(n+1)$ for all $n \geq n_{1} \geq n_{0}$. This estimate, combined with (5.25) and (5.6), shows that $H_{b}$ is HS iff $\sum\left|\alpha_{n}\right|^{2}\left\|H_{z^{n}}\right\|_{\operatorname{HS}\left(\mu_{\gamma}\right)}^{2}<\infty$, which holds if and only if $\sum\left|\alpha_{n}\right|^{2}(n+1)<\infty$, which is in turn equivalent to the assertion that $b \in B_{2}$.

Since $A z^{n}=2(\gamma+1) n z^{n}$ we see that $b \in D\left(A^{(\gamma+2) / 2}\right)$ in $\mathcal{H}^{2}$ if and only if $\sum_{n=0}^{\infty}\left|\alpha_{n}\right|^{2}\left(n^{(\gamma+2) / 2}\right)^{2}\left\|z^{n}\right\|_{L^{2}\left(\mu_{\gamma}\right)}^{2}<\infty$. But, since $\left\|z^{n}\right\|_{L^{2}\left(\mu_{\gamma}\right)}^{2}$ is on the order $n^{-(\gamma+1)}$ for large $n$, it follows that $b \in D\left(A^{(\gamma+2) / 2} \mid \mathcal{H}^{2}\left(\mu_{\gamma}\right)\right)$ if and only if $b \in B_{2}$. Thus the Besov space $B_{2}$ coincides with $D\left(A^{(\gamma+2) / 2} \mid \mathcal{H}^{2}\left(\mu_{\gamma}\right)\right)$ for all $\gamma>-1$.

Remark 5.14 (Failure of optimality in Bergman spaces). We saw in Example 4.15 that the minimum regularity condition on a holomorphic symbol $b$ of the form $\left\|e^{t A} b\right\|_{L^{2}\left(\gamma_{a}\right)}<\infty$ needed to produce a bounded Hankel operator 
on $\mathcal{H}^{2}$ in the Gaussian case, is accurately given by the minimum time requirement $e^{2 t / c} \geq 2$, specified in Theorem 4.5. In that example the constant $c$ in (2.21) is given by $c=a$. Moreover it is known that (2.21) is not satisfied for $\gamma_{a}$ for any $c<a$ even if one allows $\beta>0$.

In contrast, for the weighted Bergman spaces, there is evidence from the work of Mueller and Weissler over the interval $(-1,1),[\mathbf{M W}]$, that (2.21) may hold for all $c>0$ with a $c$ dependent $\beta:=\beta(c)$. In this case a lower bound on $t$ of the form $e^{2 t / c} \geq 2$ provides no lower bound at all. One should then expect that $H_{b}$ will be bounded on the weighted Bergman spaces if $\left\|e^{t A} b\right\|<\infty$ for any $t>0$. And in fact Example 5.13 shows that $H_{b}$ is not only bounded but also HS if the holomorphic symbol $b$ satisfies $\left\|(A+1)^{(\gamma+2) / 2} b\right\|_{L^{2}\left(\mu_{\gamma}\right)}<\infty$, which is itself a much weaker condition than one of the form $\left\|e^{t A} b\right\|_{L^{2}\left(\mu_{\gamma}\right)}<\infty$, for some $t>0$. Moreover [Pe1], see also [JPR, Theorem 12.1], has shown that boundedness of $H_{b}$ on $\mathcal{H}^{2}\left(\mu_{\gamma}\right)$ requires an even weaker condition than this.

Can the general methods of Section 4 capture such a weak sufficient condition for boundedness of $H_{b}$ ? We speculate that a knowledge of the function $\beta(c)$ might allow one to come at least as close to the known best result [Pe1], [JPR, Theorem 12.1] for the weighted Bergman spaces as we did in the Gaussian case (cf. Example 4.15).

Remark 5.15. In Example 5.13 the HS-property of $H_{b}$ cannot be characterized by a probability measure $\widetilde{\mu}$ on the unit disk $D$ as in (5.9). This follows from the fact that the moments $\left\|z^{n}\right\|_{L^{2}(\widetilde{\mu})}$ must decrease monotonically to zero for any probability measure $\widetilde{\mu}$ on $D$. But we saw that $\left\|H_{z^{n}}\right\|_{\operatorname{HS}\left(\mu_{\gamma}\right)} \nearrow \infty$. So the second inequality in (5.8) cannot hold. It seems doubtful to us that there are any pairs of measures $\mu, \widetilde{\mu}$, with smooth radial densities on the unit disk, for which (5.8) holds. But we have not been able to settle this.

Theorem 5.16 (Operator bounds for $H_{b}$ when $b$ is in $E_{\lambda}$ ). Suppose that the logarithmic Sobolev inequality (2.21) holds and that $b \in E_{\lambda}$. Then

$$
\left\|H_{b}\right\|_{\mathcal{H}^{2} \rightarrow \mathcal{H}^{2}} \leq 2^{c \lambda / 2} e^{\beta}\|b\|_{2} .
$$

If, in addition, $\lambda=\mu+\nu$ with $\mu$ and $\nu \in S$ then $H_{b}\left(E_{\nu}\right) \subset E_{\mu}$ and

$$
\left\|H_{b}\right\|_{E_{\nu} \rightarrow E_{\mu}} \leq\left(\frac{\lambda^{\lambda}}{\mu^{\mu} \nu^{\nu}}\right)^{c / 2} e^{\beta}\|b\|_{2} .
$$

The entire coefficient of $\|b\|_{2}$ is to be interpreted as 1 if $\mu$ or $\nu=0$.

Proof. By (2.24) $M(2,4)^{2}=e^{4 \beta(1 / 2-1 / 4)}=e^{\beta}$. Hence (4.11), with $r=$ 2 , gives $\left\|H_{b}\right\|_{\mathcal{H}^{2} \rightarrow \mathcal{H}^{2}} \leq e^{\beta}\left\|e^{t A} b\right\|_{2}$ where $e^{2 t / c}=2$. But $e^{t A} b=e^{t \lambda} b=$ $\left(e^{2 t / c}\right)^{c \lambda / 2}=2^{c \lambda / 2} b$ from which (5.26) follows. To prove (5.27) observe first that by Lemma 5.5 (a) one has $H_{b}\left(E_{\nu}\right) \subset E_{\mu}$. Now take $q \geq p=2$ in (2.22) 
to find

$$
\left\|e^{-t_{J} A} f\right\|_{q} \leq e^{2 \beta(1 / 2-1 / q)}\|f\|_{2} \quad \text { where } \quad e^{2 t_{J} / c}=q / 2 .
$$

If $f \in E_{\sigma}$ then $e^{-t_{J} A} f=e^{-t_{J} \sigma} f=(q / 2)^{-c \sigma / 2} f$. Hence

$$
\|f\|_{q} \leq\left(\frac{q}{2}\right)^{\frac{c \sigma}{2}} e^{2 \beta(1 / 2-1 / q)}\|f\|_{2}, \quad f \in E_{\sigma}, \quad q \geq 2 .
$$

Now let $f \in E_{\mu}, g \in E_{\nu}$ and $b \in E_{\lambda}$ where $\lambda=\mu+\nu$. In case $\mu>0$ and $\nu>0$ Equation (5.28) together with Hölders inequality yields

$$
\begin{aligned}
\left|\left\langle f, H_{b} g\right\rangle\right| & =\left|\int_{M} \bar{b} f g d \mu\right| \leq\|b\|_{q_{1}}\|f\|_{q_{2}}\|g\|_{q_{3}} \\
& \leq\left[\left(q_{1} / 2\right)^{\lambda}\left(q_{2} / 2\right)^{\mu}\left(q_{3} / 2\right)^{\nu}\right]^{c / 2} e^{\beta}\|b\|_{2}\|f\|_{2}\|g\|_{2},
\end{aligned}
$$

if $q_{1}^{-1}+q_{2}^{-1}+q_{3}^{-1}=1$ and $q_{1}, q_{2}, q_{3} \geq 2$. The choices $q_{1}=2, q_{2}=2 \lambda / \mu, q_{3}=$ $2 \lambda / \nu$ satisfy these requirements on the $q_{j}$ and yield the inequality (5.27). In case $\nu=0$ we have $g \in E_{0}$, so $g$ is a constant. Therefore $\left|\left\langle f, H_{b} g\right\rangle\right|=$ $\left|\int_{M} \bar{b} f g d \mu\right| \leq|g| \int_{M}|b f| d \mu \leq\|b\|_{2}\|f\|_{2}\|g\|_{2}$, which is (5.27) for $\nu=0$. A similar argument applies if $\mu=0$. We point out that the above choice of the $q_{j}$ actually minimizes the coefficient in (5.29). A reader can verify this by setting $x_{j}=1 / q_{j}$ and using Lagrange multipliers to maximize $x_{1}^{\lambda} x_{2}^{\mu} x_{3}^{\nu}$ subject to the condition $x_{1}+x_{2}+x_{3}=1$.

In view of Theorem 4.11 it is of interest to know when the operator $(A+1)^{-s}$ is trace class on $\mathcal{H}^{2}$. Of course if $\mathcal{H}^{2}$ is finite dimensional, as in Example 3.18, then $(A+1)^{-s}$ is always trace class on $\mathcal{H}^{2}$. This case is not of interest to us because $\mathcal{R}$ cannot be an algebra in such a circumstance. (See the discussion in Example 3.18.) If $\mathcal{H}^{2}$ is infinite dimensional then we have the following simple dependence on the dimension of $M$.

Lemma 5.17. Let $(M, g, \mu)$ be one of the examples of Section 3. Assume that $\operatorname{dim} \mathcal{H}^{2}=\infty$. If complex dimension $M=m$ then

$$
\text { trace }_{\mathcal{H}^{2}}(A+1)^{-s}<\infty \quad \text { if and only if } s>m \text {. }
$$

Proof. In all the examples of Section 3 the Dirichlet form operator $A$ has the form $A=C\left[\sum_{k=1}^{m} z_{k} \partial / \partial z_{k}\right]$ on $\mathcal{H}^{2}$ for some constant $C>0$ where $m$ is the complex dimension of $M$. The meaning of this expression is clear when $M=\mathbb{C}^{m}$ or some open subset of $\mathbb{C}$ and has also been explained in Example 3.9 when $M$ is the Riemann surface for $z^{1 / n}$. In all cases the spectrum of $A \mid \mathcal{H}^{2}$ is the same as for a harmonic oscillator, namely $\{0, C, 2 C, \ldots\}$. Moreover the multiplicity of these eigenvalues depends in a simple way on the dimension. It will suffice to focus on the case of Example 3.1, in which $M=\mathbb{C}^{m}$, all other cases being similar. The eigenfunctions of $A$ in $\mathcal{H}^{2}$ are the polynomials $z_{1}^{k_{1}} \ldots z_{m}^{k_{m}}$ with corresponding eigenvalue $C\left(k_{1}+\cdots+k_{m}\right)$, cf. Eq. (3.12). Hence $\operatorname{trace}_{\mathcal{H}^{2}}(A+1)^{-s}=$ $\sum_{k_{1}, \ldots, k_{m} \geq 0}\left(1+C\left(k_{1}+\cdots+k_{m}\right)\right)^{-s}=\sum_{j=0}^{\infty} B(m, j)(1+C j)^{-s}$ where $B(m, j)$ 
is the number of partitions: $k_{1}+\cdots+k_{m}=j$. By inscribing and circumscribing squares in and around the simplex $\left\{k_{1}+\cdots+k_{m}=j\right.$; all $\left.k_{j} \geq 0\right\}$ one sees that $B(m, j)$ is on the order of $j^{m-1}$ for large $j$. Hence trace $\mathcal{H}^{2}(A+1)^{-s}<\infty$ if and only if $m-1-s<-1$. This proves (5.30).

In the next example we will combine (5.30) with Corollary 4.12.

Example 5.18 (HS class and non-Gaussian measures on $\mathbb{C}^{m}$ ). Refering for notation to Example 3.1 let us assume that the logarithmic Sobolev inequality (2.21) holds. This was shown in Theorem 3.2 to be the case in many instances. By (5.30) $(A+1)^{-s}$ is HS if and only if $2 s>m$. Thus we may conclude from Corollary 4.12 that in these examples $H_{b}$ is HS if $b$ is given by (4.19) and $2 s>m$.

But we must note that unless the constant $c$ in (2.21) is the smallest one for which (2.21) holds (for some $\beta \geq 0$ ), the factor $(A+1)^{-s}$ is unnecessary to reach the conclusion of Corollary (4.12). The reason for this is that if (2.21) holds for $c$ and for $c_{0}<c$ (different $\beta \mathrm{s}$ ) and $t_{J}^{0}\left(2,2 r^{\prime}\right)$ corresponds to $c_{0}$ as in (2.23) then $t_{J}=t_{J}^{0}+\epsilon$ for some $\epsilon>0$. Hence $b \equiv e^{-t_{J} A} \varphi=e^{-\epsilon A} e^{-t_{J}^{0} A} \varphi$. But $e^{-t_{J}^{0} A} \varphi$ is the symbol of a bounded Hankel operator while $e^{-\epsilon A}$ is HS class for all $\epsilon>0$ because trace $\mathcal{H}^{2} e^{-\epsilon A}=\left(\prod_{k \geq 0} e^{-\epsilon C k}\right)^{m}<\infty$ for all $\epsilon>0$. So $H_{b}$ is already HS by Corollary 4.7 .

Example 5.19 (Gaussian case. Comparison with [JPR]). Here we will show that the general methods of Section 4 do not reproduce the known best results on the Hilbert-Schmidt character of $H_{b}$ in the Gaussian case. For $\mu=\gamma_{a}$ the smallest allowed value of $c$ in (2.21) is $c=a$. Take $r=2$ in Corollary 4.12 as we did in the discussion of boundedness in Example 4.15. If $\varphi \in \mathcal{H}^{2}$ and $b=e^{-t A} \varphi$ with $t=t_{J}(2,4)$ then $H_{b}$ is bounded on $\mathcal{H}^{2}$ and no smaller $t$ than this will assure this (see Example 4.15). Then Corollary 4.7 assures that $e^{-\epsilon A} b$ is the symbol of a HS Hankel operator for any $\epsilon>0$ because $e^{-\epsilon A}$ is HS. Theorem 4.11 combined with Lemma 5.17 gives a more refined result, namely $(A+1)^{-r} b$ is the symbol of a HS Hankel operator if $2 r>m$. But in fact it is known, [JPR, Theorem 7.8], (and reproved in (5.19) and again in (6.11)) that $b$ is already the symbol of a HS operator. It doesn't need any further smoothing by $e^{-\epsilon A}$ or even by $(A+1)^{-r}$. Thus, as in the case of boundedness, the general methods of Section 4 do not reproduce the known weakest conditions that assure that $H_{b}$ is HS.

Remark 5.20. There is yet another way to estimate $\left\|H_{b}\right\|_{\mathrm{HS}}$ when $b \in E_{\lambda}$. In view of Lemma 5.5 it is easy to show that if $b \in E_{\lambda}$ then $\left\|H_{b}\right\|_{\mathrm{HS}}^{2} \leq$ $\sum_{\nu \leq \lambda}\left\|H_{b}\right\|_{E_{\nu} \rightarrow E_{\lambda-\nu}} \cdot \operatorname{dim}\left(E_{\lambda-\nu}\right)$ with equality holding if $\operatorname{dim} E_{\mu}=1$ for all eigenvalues $\mu$. By using the estimates (5.27) one can show that in the class of non-Gaussian measures of Example 3.1, in dimension $m=1$, the symbol $b=(A+1)^{-1 / 2} e^{-t_{J}(2,4) A} \varphi$ gives a HS operator on $\mathcal{H}^{2}(\mu)$ when $\varphi \in L^{2}(\mu)$. This is a slight improvement over Example 5.18 (for $m=1$ ), which requires 
a factor $(A+1)^{-s}$ for some $s>1 / 2$. In any case this improvement still does not capture the known best result in the Gaussian case.

\section{The reproducing kernel and the Dirichlet form.}

We return now to the general theory and explore the connections between the Dirichlet form and the reproducing kernel. The Dirichlet form operator $A$ relates well to the reproducing kernel for the Hilbert space $\mathcal{H}^{2}$.

We will assume that the Standard Conditions of Notation 2.3 hold throughout this section. But we do not need to assume that $\mathcal{R}$ is an algebra.

Notation 6.1 (The reproducing kernel). For each point $z \in M$ the linear functional $\mathcal{H}^{2} \ni f \mapsto f(z)$ is continuous and is therefore given by the inner product with a unique function $K_{z}(\cdot) \in \mathcal{H}^{2}: f(z)=\left\langle f, K_{z}\right\rangle$ for $f \in \mathcal{H}^{2}$. $K_{z}(\cdot)$ is the reproducing kernel for $\mathcal{H}^{2}$. Denote by $P_{\mathcal{H}^{2}}$ the orthogonal projection of $L^{2}$ onto $\mathcal{H}^{2}$. Since $K_{z}$ is orthogonal to $\left(\mathcal{H}^{2}\right)^{\perp}$ we have

$$
\left(P_{\mathcal{H}^{2}} f\right)(z)=\left\langle f, K_{z}\right\rangle \quad \forall f \in L^{2}(\mu) .
$$

$e^{-t A}$ leaves $\mathcal{H}^{2}$ invariant for all $t>0$ and is Hermitian. It therefore commutes with $P_{\mathcal{H}^{2}}$. So the unitary operators $e^{i s A}$ also commute with $P_{\mathcal{H}^{2}}$, for all real $s$.

Theorem 6.2. For $t \geq 0$ and all real $s$ there holds

$$
K_{z}\left(e^{-t X} w\right)=\left(e^{-t A} K_{z}\right)(w)=K_{e^{-t X} z}(w)
$$

and

$$
K_{z}\left(e^{-s Y} w\right)=\left(e^{-i s A} K_{z}\right)(w)=K_{e^{s Y}}(w) .
$$

Proof. The first equality in each line is a different special case of (2.19). Now for any function $f \in \mathcal{H}^{2}$ we have

$$
\left\langle f, K_{e^{-t X} z}\right\rangle=f\left(e^{-t X} z\right)=\left(e^{-t A} f\right)(z)=\left\langle e^{-t A} f, K_{z}\right\rangle=\left\langle f, e^{-t A} K_{z}\right\rangle .
$$

Since $\left(e^{-t A} K_{z}\right)(\cdot)$ and $K_{e^{-t X}}(\cdot)$ are both in $\mathcal{H}^{2}$ the second equality in (6.2) follows. Similarly, $\left\langle f, K_{e^{s Y}}\right\rangle=f\left(e^{s Y} z\right)=\left(e^{i s A} f\right)(z)=\left\langle e^{i s A} f, K_{z}\right\rangle=$ $\left\langle f, e^{-i s A} K_{z}\right\rangle$, which yields the second equality in (6.3).

Corollary 6.3. Let $t \geq 0$. The operator $e^{-t A} P_{\mathcal{H}^{2}}$ on $L^{2}$ is given by the integral operator with kernel

$$
(z, w) \mapsto K_{e^{-t X} z}(w) .
$$

Proof. If $f \in L^{2}(\mu)$ then

$$
\left(e^{-t A} P_{\mathcal{H}^{2}} f\right)(z)=\left(P_{\mathcal{H}^{2}} f\right)\left(e^{-t X} z\right)=\left\langle f, K_{e^{-t X} z}\right\rangle .
$$


Remark 6.4. Several other identities follow from Theorem 6.2 and Corollary 6.3. For example the operator $e^{-(t+s) A} P_{\mathcal{H}^{2}}$ on $L^{2}$ is given by the integral operator with kernel $(z, w) \mapsto K_{e^{-s X}}\left(e^{-t X} w\right)$ for $t$ and $s$ nonnegative. Also the operator $e^{i s A} P_{\mathcal{H}^{2}}$ is given by the kernel $(z, w) \mapsto K_{e^{s Y}}(w)$. Since $e^{-2 t A} P_{\mathcal{H}^{2}}$ is a nonnegative operator and $K$ is jointly continuous we have, with the aid of Mercer's theorem, the interesting identity

$$
\int_{M} K_{e^{-t X} z}\left(e^{-t X} z\right) d \mu(z)=\operatorname{trace}_{L^{2}}\left(e^{-2 t A} P_{\mathcal{H}^{2}}\right)=\operatorname{trace}_{\mathcal{H}^{2}}\left(e^{-2 t A}\right)
$$

if the right side is finite.

The following result shows that $K_{z}$ has strong regularity properties.

Theorem 6.5. If $X$ is two-sided complete then

$$
K_{z} \in D\left(e^{t A} \mid \mathcal{H}^{2}\right) \quad \forall z \in M \text { and } \forall t \geq 0 .
$$

If, in addition, the logarithmic Sobolev inequality (2.21) holds then

$$
K_{z} \in \cap_{p<\infty} L^{p}(\mu) .
$$

In this case, for $p>1$, the reproduction formula extends to $\mathcal{H}^{p}$ :

$$
\int_{M} f(w) \overline{K_{z}(w)} d \mu(w)=f(z), \quad z \in M, f \in \mathcal{H}^{p} .
$$

Proof. If $X$ is two-sided complete then by (6.2) we have

$$
K_{z}=K_{e^{-t X}} e^{t X} z=e^{-t A} K_{e^{t X}} .
$$

This proves $(6.5)$. For any $p \in(2, \infty)$ there exists, by $(2.22)$, a $t>0$ such that $e^{-t A}: \mathcal{H}^{2} \rightarrow \mathcal{H}^{p}$ is bounded. Choosing such a $t,(6.8)$ shows that $K_{z}(\cdot)$ is in $\mathcal{H}^{p}$. This proves (6.6). Now (6.7) holds for $f \in \mathcal{H}^{p}$ if $2 \leq p<\infty$ because $\mathcal{H}^{p} \subset \mathcal{H}^{2}$ in this case. If $1<p<2$ then $\mathcal{H}^{2}$ is dense in $\mathcal{H}^{p}$ by the definition of $\mathcal{H}^{p}$, (cf. (2.10)). Since $K_{z} \in L^{p^{\prime}}$ (6.7) extends by continuity of both sides to all of $\mathcal{H}^{p}$.

Example 6.6 (Gauss measure on $\mathbb{C}^{m}$ ). In the notation of Example 2.4 the reproducing kernel is $K_{z}(w)=e^{\bar{z} \cdot w / 2 a}$. In view of the known flows $e^{-t X}$ (respectively $e^{-s Y}$ ) from Example 2.4, the equality of the first and third terms in (6.2), respectively (6.3), are in agreement with the Gaussian case. (6.6) also clearly holds in the Gaussian case.

Remark 6.7. Under the hypotheses of Theorem 6.5 and for $1<p<\infty$ the linear functional $L^{p} \ni g \mapsto h(z):=\int_{M} g(w) \overline{K_{z}(w)} d \mu(w)$ is clearly continuous for each $z \in M$. Moreover $h$ is holomorphic. But (6.7) does not imply that the map $g \mapsto h$ is necessarily continuous from $L^{p}$ into $\mathcal{H}^{p}$ when $p \neq 2$. For $p \neq 2$ there are other reproducing kernels that are appropriate for $\mathcal{H} L^{p}\left(\gamma_{a}\right)$ in the Gaussian case. See [JPR, Theorem 7.1] and [GW]. 
Remark 6.8 (Integral representation of $H_{b}$ ). The representation of the Hankel operator $H_{b}$ as an integral operator is well-known in the classical context (see e.g., [Zhh, Section 7.6]) and over Gaussian spaces (see [JPR], Section 10). Our structures allow a similar representation. We assume that $\mathcal{R}$ is an algebra in this remark in order to give meaning to the Hankel operator $H_{b}$. Suppose that $b \in L^{2}(\mu)$ and that $\Gamma_{b}$ is continuous on $\mathcal{R} \times \mathcal{R}$ in $L^{2}$ norm (cf. (4.1)). Then $H_{b}$ exists as a bounded operator on $\mathcal{H}^{2}$. Let

$$
k_{b}(z, w)=\left(H_{b} K_{z}\right)(w) .
$$

Then

(i) $k_{b}(z, w)=k_{b}(w, z) \forall z, w \in M$.

(ii) $k_{b}(z, \cdot) \in \mathcal{H}^{2}$ for each $z \in M$.

(iii) $\left(H_{b} f\right)(z)=\left\langle k_{b}(z, \cdot), f\right\rangle \forall f \in \mathcal{H}^{2}$.

(iv) $\left\|H_{b}\right\|_{\mathrm{HS}}^{2}=\int_{M} \int_{M}\left|k_{b}(z, w)\right|^{2} d \mu(z) d \mu(w)$.

Proof. $\left(H_{b} K_{z}\right)(w)=\left\langle H_{b} K_{z}, K_{w}\right\rangle=\left\langle H_{b} K_{w}, K_{z}\right\rangle=\left(H_{b} K_{w}\right)(z)$ by (4.12). This proves (i). (ii) follows from the definition (6.9). (iii) holds for $f=K_{\xi}$ because $\left(H_{b} K_{\xi}\right)(z)=k_{b}(\xi, z)=k_{b}(z, \xi)=\left\langle k_{b}(z, \cdot), K_{\xi}\right\rangle$. Thus (iii) holds for any finite linear combination of the $K_{\xi}$. Since these functions are dense in $\mathcal{H}^{2}$ and both sides of (iii) are continuous in $f$ in $\mathcal{H}^{2}$ norm (iii) follows. Finally, note that the integral operator $L^{2} \ni f \mapsto(T f)(z)=\left\langle k_{b}(z, \cdot), f\right\rangle$ annihilates $\left(\mathcal{H}^{2}\right)^{\perp}$. Hence $\operatorname{trace}_{L^{2}}\left(T^{*} T\right)=\operatorname{trace}_{\mathcal{H}^{2}}\left(H_{b}^{*} H_{b}\right)$. This proves (iv).

Example 6.9 (Gauss measure on $\mathbb{C}^{m}$ ). We amplify here a little bit on [JPR, Theorem 10.1]. We may write, in general, $k_{b}(z, w)=\left\langle H_{b} K_{z}, K_{w}\right\rangle$ and then, informally, as $\left\langle b, K_{z} K_{w}\right\rangle$. Since $b$ has only been assumed to be in $L^{2}$ we would need to know that $K_{z} K_{w} \in L^{2}$ in order for this expression to be well-defined. In our general seting we would therefore need to know that $K_{z} \in L^{4}(\mu)$ for each $z$. Since $\left\langle H_{b} g, f\right\rangle=\langle b, g f\rangle$ for all $f, g \in \mathcal{R}$ the useful identity

$$
k_{b}(z, w)=\left\langle b, K_{z} K_{w}\right\rangle
$$

would then follow if we knew also that $\mathcal{R}$ was dense in $\mathcal{H}^{4}$. This clearly holds in the Gaussian case and in fact, when the logarithmic Sobolev inequality (2.21) holds, $\mathcal{R}$ is dense in all $\mathcal{H}^{p}, 0<p<\infty$, [G3, Theorem 2.17]. Restricting attention now to the Gaussian case, suppose that $b \in \mathcal{H}^{2}\left(\mathbb{C}^{m}, \gamma_{a}\right)$ (which happens to equal to $\mathcal{H} L^{2}$ in this case, cf. Remark 2.5). Then, since $K_{z} K_{w}=K_{z+w}$, (6.10) gives $k_{b}(z, w)=b(z+w)$, which has already been pointed out in [JPR, Theorem 10.1]. Item (iv) in Remark 6.8 now gives $\left\|H_{b}\right\|_{\mathrm{HS}}^{2}=\int_{M} \int_{M}|b(z+w)|^{2} d \gamma_{a}(z) d \gamma_{a}(w)=\int_{M}|b(\zeta)|^{2} d\left(\gamma_{a} * \gamma_{a}\right)(\zeta)$. Hence

$$
\left\|H_{b}\right\|_{\mathrm{HS}\left(\gamma_{a}\right)}^{2}=\|b\|_{L^{2}\left(\gamma_{2 a}\right)}^{2} \quad b \in \mathcal{H}^{2}\left(\gamma_{a}\right) .
$$

This provides a less computational proof of the identity (5.19). Any functional relation of the form $K_{z} K_{w}=K_{F(z, w)}$ in $\mathcal{H} L^{2}(M, \mu)$ would similarly 
give an identity $\left\|H_{b}\right\|_{\mathrm{HS}(\mu)}=\|b\|_{L^{2}(\widetilde{\mu})}$ with $\widetilde{\mu}=F_{*}(\mu \otimes \mu)$. But we know of no examples of such a functional relation other than for Gauss measure.

Remark 6.10 (Finite rank operators). The finite rank Hankel operators have been characterized in the classical settings. See e.g., [JPR, Section 14], [Ro1], [Ro2]. It is found that a finite rank symbol is always a finite linear combination of derivatives of the reproducing kernel at different points. In our present setting it is reasonable to expect that the analog of this characterization should take the following form. (Assume here that $\mathcal{R}$ is an algebra so that we can discuss Hankel operators.) Let $Z_{1}, \ldots, Z_{n}$ be holomorphic complex vector fields of type $(1,0)$. For example we could allow all $Z_{j}=Z$, with $Z$ defined as in (2.15). Fix $z \in M$. Define a bilinear functional $\mathcal{R} \ni f, g \mapsto \Gamma(f, g):=\left\langle Z_{1} \ldots Z_{n}(f g), K_{z}\right\rangle=\left[Z_{1} \ldots Z_{n}(f g)\right](z)$. Then the kernel of $\Gamma,\{g \in \mathcal{R}: \Gamma(f, g)=0 \forall f \in \mathcal{R}\}$, is cofinite dimensional in $\mathcal{R}$ because it is determined by the zeros of $g$ and its derivatives $Z_{i_{1}} \ldots Z_{i_{k}} g$ at $z$. If one defines $Z_{j}^{*}$ as the adjoint in $\mathcal{H}^{2}$ then (ignoring domains of these unbounded operators for this informal discussion) we have $\Gamma(f, g)=\left\langle f g, Z_{n}^{*} \ldots Z_{1}^{*} K_{z}\right\rangle$. So $\Gamma=\Gamma_{b}$ where $b=Z_{n}^{*} \ldots Z_{1}^{*} K_{z} . b$ is therefore the symbol of a finite rank Hankel operator. In general $Z_{j}^{*}$ will be a first order differential operator with a zeroth order term, followed by $P_{\mathcal{H}^{2}}$. But with $Z$ defined by (2.15) one has simply $Z^{*}=Z$ on $\mathcal{H}^{2}$ because $A$ is self-adjoint on $\mathcal{H}^{2}$. For the one complex dimensional Riemann surface of Example 3.4 it seems reasonable to conjecture that every finite rank symbol is of the form $b(\cdot)=\sum_{k=1}^{N} a_{k} Z^{n_{k}} K_{z_{k}}(\cdot)$ for some constants $a_{k}$.

Remark 6.11 (Reverse estimates). The reproducing kernel is potentially useful for establishing necessary conditions on a symbol $b$ in order for $H_{b}$ to be bounded. Thus suppose that $b \in \mathcal{H}^{2}$. Starting with (6.9) we have

$$
\left\|k_{b}(z, \cdot)\right\|_{2}=\left\|H_{b} K_{z}\right\|_{2} \leq\left\|H_{b}\right\|_{o p}\left\|K_{z}\right\|_{2} \text {. }
$$

So

$$
\left\|H_{b}\right\|_{o p}^{2} \geq \int_{M}\left\|K_{z}\right\|_{2}^{-2}\left\|k_{b}(z, \cdot)\right\|_{2}^{2} d \rho(z)
$$

for any probability measure $\rho$ on $M$. This yields a useable lower bound on $\left\|H_{b}\right\|_{o p}$ in the Gaussian case, $M=\mathbb{C}^{m}, \mu=\gamma_{a}$, as follows. Choose $\rho=\gamma_{s}$ with large $s$. Suppose that $u^{-1}=a^{-1}+s^{-1}$. Since $\left\|K_{z}\right\|_{L^{2}\left(\gamma_{a}\right)}^{2}=K_{z}(z)=$ $e^{|z|^{2} / 2 a}$, we have

$$
\begin{aligned}
\left\|H_{b}\right\|_{o p}^{2} & \geq \int_{\mathbb{C}^{m}}\left(\int_{\mathbb{C}^{m}}|b(z+w)|^{2} d \gamma_{a}(w)\right) e^{-|z|^{2} / 2 a} d \gamma_{s}(z) \\
& =(u / s)^{m} \int_{\mathbb{C}^{m}} \int_{\mathbb{C}^{m}}|b(z+w)|^{2} d \gamma_{a}(w) d \gamma_{u}(z) \\
& =(u / s)^{m}\|b\|_{L^{2}\left(\gamma_{a+u}\right)}^{2} .
\end{aligned}
$$


So if $\left\|H_{b}\right\|_{o p}<\infty$ then $\|b\|_{L^{2}\left(\gamma_{a+u}\right)}<\infty$ whenever $0<s<\infty$. I.e., whenever $u<a$. In view of (4.21) we see that $\left\|H_{b}\right\|_{o p}<\infty$ implies that $\left\|e^{t A} b\right\|_{L^{2}\left(\gamma_{a}\right)}<$ $\infty$ whenever $e^{2 t / a}<2$. Thus the inequality (4.3) (with $p=q=r=2$ ) gives the best value of $t$ in the Gaussian case. We have already noted this in Example 4.15, using there the deep results of [JPR].

Acknowledgment. We thank the referee for his valuable and detailed suggestions. They helped a lot to make our manuscript more intelligible.

\section{References}

[AS] S. Aida and I. Shigekawa, Logarithmic Sobolev inequalities and spectral gaps: Perturbation theory, J. Funct. Anal., 126 (1994), 448-475.

[AHS] S. Albeverio, R. Hoegh-Krohn and L. Streit, Energy forms, Hamiltonians, and distorted Brownian paths, J. Math. Phys., 18 (1977), 907-917, MR 56 \#4564, Zbl 0368.60091.

[Ba] V. Bargmann, On a Hilbert space of analytic functions and an associated integral transform, Part I, Comm. Pure and Appl. Math., 24 (1961), 187-214, MR 28 \#486, Zbl 0107.09102.

[BRZ] V. Bogachev, M. Rockner and T.S. Zhang, Existence and uniqueness of invariant measures: An approach via sectorial forms, Appl. Math. Optim., 41 (2000), 87-109, MR 2000k:31014, Zbl 0953.31003.

[BH] N. Bouleau and F. Hirsch, Dirichlet Forms and Analysis on Wiener Space, W. de Gruyter, 1991, MR 93e:60107, Zbl 0748.60046.

[C] E. Carlen, Some integral identities and inequalities for entire functions and their applications to the coherent state transform, J. of Funct. Anal., 97 (1991), 231-249, MR 92i:46025, Zbl 0743.46018.

[Ci] F. Cipriani, Sobolev-Orlicz imbeddings, weak compactness and spectrum, J. of Funct. Anal., 177 (2000), 89-106, MR 2001m:46069, Zbl 0974.46036.

[Da1] E.B. Davies, One-Parameter Semigroups, Academic Press, 1980, MR 82i:47060, Zbl 0457.47030 .

[Da2] - Heat Kernels and Spectral Theory, Cambridge Univ. Press, 1989, MR 90e:35123, Zbl 0699.35006.

[DS] N. Dunford and J.T. Schwartz, Linear Operators, Part 2, Wiley Interscience, New York, 1963, MR 32 \#6181, Zbl 0128.34803.

[Fu1] M. Fukushima, Energy forms and diffusion processes, in 'Mathematics and Physics,' 1, Ed. L. Streit, World Scientific, Singapore and Philadelphia, (1985), 65-97, MR 87m:60176, Zbl 0676.60071.

[Fu2] M. Fukushima, Y. Oshima and M. Takeda, Dirichlet forms and symmetric Markov processes, de Gruyter Studies in Mathematics, 19, Walter de Gruyter and Co., Berlin, 1994, MR 96f:60126, Zbl 0838.31001.

[GW] D.J.H. Garling and P. Wojtaszczyk, Some Bargmann spaces of analytic functions, in 'Function Spaces, The Second Conference,' Ed. Krzysztof Jarosz, Lecture Notes in Pure and Applied Mathematics, 172, Dekker, (1995), 123-138, MR 96h:46032, Zbl 0848.46012. 
[GrR] I.S. Gradshteyn and I.M. Ryzhik, Table of Integrals, Series, and Products, Academic Press, 1980, MR 87j:00023, Zbl 0521.33001.

[G1] L. Gross, Logarithmic Sobolev inequalities, Amer. J. of Math., 97 (1975), 1061-1083, MR 54 \#8263, Zbl 0318.46049.

[G2] Logarithmic Sobolev inequalities and contractivity properties of semigroups, in 'Dirichlet Forms', G. Dell'Antonio and U. Mosco, Eds., Varenna 1992, Lecture Notes in Mathematics, 1563, Springer-Verlag, Berlin, 1993, MR 95h:47061, Zbl 0812.47037.

[G3] - Hypercontractivity over complex manifolds, Acta Math., 182 (1999), 159206, MR 2001c:58034.

[G4] Strong hypercontractivity and relative subharmonicity, J. Funct. Anal., 190 (2002), 38-92.

[GR] L. Gross and O. Rothaus, Herbst inequalities for supercontractive semigroups, J. of Math. of Kyoto Univ., 38 (1998), 295-318, MR 99i:47074, Zbl 0928.47032.

[HR] F. Holland and R. Rochberg, Bergman kernels and Hankel forms on generalized Fock spaces, in 'Function spaces', K. Jarosz, Ed., Contemporary Mathematics, 232 (1999), 189-200, MR 2000b:46050, Zbl 0946.47016.

[HS] R. Holley and D.W. Stroock, Logarithmic Sobolev inequalities and stochastic Ising models, J. Stat. Phys., 46 (1987), 1159-1194.

[Ja1] S. Janson, On hypercontractivity for multipliers on orthogonal polynomials, Ark. Mat., 21 (1983), 97-110, MR 85g:47070, Zbl 0516.42022.

[Ja2] - Hankel operators on Bergman spaces with change of weight, Math. Scand., 71 (1992), 267-276, MR 94a:47042, Zbl 0792.47028.

[JPR] S. Janson, J. Peetre and R. Rochberg, Hankel forms and the Fock space, Rev. Mate. Ibero., 3 (1987), 61-138, MR 91a:47029, Zbl 0704.47022

[Ka] T. Kato, Perturbation Theory for Linear Operators, Springer, 1995, MR 96a:47025, Zbl 0836.47009.

[MR] Z.-M. Ma and M. Röckner, Introduction to the Theory of (Non-Symmetric) Dirichlet Forms, Springer-Verlag, 1992, MR 94d:60119, Zbl 0826.31001.

[MW] C.E. Mueller and F. Weissler, Hypercontractivity for the heat semigroup for ultraspherical polynomials and on the n-sphere, J. Funct. Anal., 48 (1982), 252-283, MR 83m:47036, Zbl 0506.46022.

[OR] O.A. Oleinik and E.V. Radkevic, Second Order Equations with Nonnegative Characteristic Form, AMS, Plenum Press, 1973, MR 56 \#16112.

[Pa] J.R. Partington, An Introduction to Hankel Operators, Cambridge, 1988, MR 90c:47047, Zbl 0668.47022.

[P] J. Peetre, Generalizations of Hankel Operators, Nonlinear Analysis, Function Spaces and Appl., 3, Teubner, 1986, MR 89d:47047, Zbl 0639.47020.

[PR] J. Peetre and R. Rochberg, Higher order Hankel forms, in 'Multivariable Operator Theory', Curto, Douglas, Pincus and Salinas, Eds., Contemporary Mathematics, 185 (1995), 283-306, MR 96g:47020, Zbl 0944.47019.

[Pe1] V.V. Peller, Vectorial Hankel operators, commutators and related operators of the Schatten-von Neumann $S_{p}$, Integral Equations and Operator Theory, 5 (1982), 244-272, MR 83f:47024, Zbl 0478.47014. 
[Pe2] - Hankel operators and multivariate stationary processes, in 'Operator Theory: Operator Algebras and Applications, Part 1,' Proc. Symp. Pure Math., 51, A.M.S., Providence, 1990, 357-371, MR 91m:47034, Zbl 0712.47020.

[Pe3] _ An excursion into the theory of Hankel operators, in 'Holomorphic Spaces,' eds. S. Axler et al., Cambridge, 1998, 65-120, MR 99e:47033.

[PK] V.V. Peller and S.V. Khrushchev, Hankel operators, best approximation, and stationary Gaussian processes, Russ. Math. Surv., 37(1) (1982), 61-144, MR 84e:47036, Zbl 0505.60043.

[Po1] S.C. Power, Hankel operators on Hilbert space, Bul. Lond. Math. Soc., 12 (1980), 422-442, MR 82a:47030, Zbl 0446.47015.

[Po2] _ Hankel Operators on Hilbert Space, Pitman Research Notes, 64, 1982, MR 84e:47037, Zbl 0489.47011.

[RS] M. Reed and B. Simon, Methods of Modern Mathematical Physics, 1, Academic Press, 1972, MR 58 \#12429a, Zbl 0242.46001.

[Ro1] R. Rochberg, Higher-order Hankel forms and commutators, in 'Holomorphic Spaces,' eds. S. Axler et al., Cambridge, 1998, MR 2000g:47032.

[Ro2] , A Kronecker theorem for higher order Hankel forms, Proc. Amer. Math. Soc., 123 (1995), 3113-3118, MR 95m:47042, Zbl 0860.47016.

[Se] I.E. Segal, The complex-wave representation of the free boson field, Topics in functional analysis (essays dedicated to M. G. Kreln on the occasion of his 70th birthday), Academic Press, New York, (1978), 321-343, MR 82d:81069, Zbl 0471.22024.

[Sj] P. Sjögren, Un contre-exemple pour le noyau reproduisant de la mesure gaussienne dans le plan complexe, Séminaire Paul Krée (Equations aux dérivées partielles en dimension infinie) 1975/76, Paris, MR 58 \#17808, Zbl 0357.28009.

[Wa] F.-Yu Wang, Functional inequalities for empty essential spectrum, J. Funct. Anal., 170 (2000), 219-245, MR 2001a:58043, Zbl 0946.58010.

[Zh] K. Zhu, Operator Theory in Function Spaces, M. Dekker Inc., New York, Basel, 1990, MR 92c:47031, Zbl 0706.47019.

Received May 22, 2000 and revised May 7, 2001. The first author was supported by the Alexander von Humboldt Foundation.

FAKUltätT FÜR MATHEMATIK UND INFORMATIK

UNIVERSITÄT MANNHEIM

D-68131 Mannheim, Germany

E-mail address: deck@math.uni-mannheim.de

Department of Mathematics

CORNELl UNIVERSity

ITHACA, NY 14853

E-mail address: gross@math.cornell.edu 